\title{
On Entropy and Monotonicity for Real Cubic Maps
}

\author{
John Milnor and Charles Tresser ${ }^{1}$ \\ with an appendix by Adrien Douady and Pierrette Sentenac
}

\section{Contents}

\section{Introduction}

2. Piecewise monotone maps and kneading theory

3. Parametrization of polynomial maps

4. Topological entropy and periodic orbits

5. The stunted sawtooth family

6. Contractibility of isentropes for the stunted sawtooth family

7. Bones in $P^{2}$

8. Monotonicity, intersections of bones, and the period $n$-skeleton

9. From connected bones to connected isentropes

Appendix A. (by A. Douady and P. Sentenac): Characterization of a polynomial by its critical values

Appendix B: Tight symbol sequences and Thurston's theorem

Appendix C. Monotonicity versus antimonotonicity

References

\section{$\S 1$. Introduction}

Consider a continuous map $f$ from a closed interval $I$ to itself. In the simplest cases, $I$ is the union (in a not necessarily unique way) of sub-intervals where $f$ is monotone. The minimal number of sub-intervals required (called the lap number) is a rough measure of the complexity of the map $f$. If now we think of $f$ as generating a dynamical system, a quantitative description of the dynamic complexity of $f$ is obtained by measuring the rate of exponential growth of the lap numbers of the successive iterates $f^{\circ 1}=f, f^{\circ 2}=f \circ f, \ldots$ of the map $f$. This growth rate is clearly invariant under a continuous change of coordinate, and turns out to be equal to a measure of dynamic complexity used in a much more general context and known as the topological entropy $h(f)$.

Maps on the interval provide the simplest examples to illustrate the problem of understanding how dynamic complexity evolves under deformations of a dynamical system. However, even the basic problem of comparing the topological entropies of two smooth interval maps which are close to each other is only partly understood. The best results so far

\footnotetext{
${ }^{1}$ Partially supported by NSF grant DMS-97-04867.
} 
have been obtained in the more specific case of polynomial maps, where one can use complex analysis methods in the study of the associated complex polynomial maps. The theory is most complete in the particular case of the family of quadratic maps $Q_{v}(x)=4 v x(1-x)$, parametrized by the critical value $0 \leq v \leq 1$. It has been known for some time that the topological entropy $h\left(Q_{v}\right)$ is a (non-strictly) increasing continuous function of the parameter $v$, with $h\left(Q_{0}\right)=0$ and $h\left(Q_{1}\right)=\log 2$. All known proofs of the monotonicity of topological entropy for quadratic maps use complex analytic methods. (Compare [D], [DH2], [MvS2], $[\mathrm{MTh}]^{2}$.) The particular proof which we will generalize is based on the following ideas. These maps $Q_{v}$ have a single critical point $c=\frac{1}{2}$ for $v \neq 0$, and there are a countable infinity of values of $v \in(0,1]$ such that $c$ belongs to a periodic orbit. The restriction of $Q_{v}$ to any periodic orbit $x_{1}<\cdots<x_{p}$ can be described combinatorially by the cyclic permutation o of $\{1, \ldots, p\}$ which we call its order type, defined by the property that $Q_{v}\left(x_{i}\right)=x_{\mathbf{o}(i)}$. Thurston showed that each order type for a periodic critical orbit which occurs in this way for some $Q_{v}$, occurs for a single value of $v$ only. (Compare $\S 8$ and Appendix B.) This fact is used in [MTh] to prove monotonicity. It has been recognized for a long time that for each quadratic map with a periodic critical orbit of period $p$ there exists a countable infinity of quadratic maps with the same topological entropy and with a critical orbit whose period is some multiple of $p$. Thus the monotonicity of the correspondence $v \mapsto h\left(Q_{v}\right)$ cannot be strict.

On the other hand, it has been proved more recently that any value of the topological entropy in $[0, \log 2]$ which cannot be achieved by a map having a periodic critical orbit, is realized for a single value of $v$. ([Gs], [L2].) This result is a special case of the Generic Hyperbolicity Conjecture, which states that every rational map can be approximated arbitrarily closely by a rational map (real if the original map is real, and a polynomial if the original is a polynomial) such that the orbits of all critical points converge to attracting periodic orbits. (For an early version on this conjecture, see Fatou [F].)

One would like to understand as much as possible of the global bifurcation theory for polynomials of higher degree. But there, even the relevant concepts are harder to isolate because families of degree $d$ polynomials depend naturally on $d-1$ parameters. (In fact we will usually work with the integer $m=d-1$.) For each fixed degree $d$ and for each fixed sign \pm 1 we consider the family of all degree $d$ polynomial maps from the interval $I=[a, b]$ to itself, with all $d-1$ critical points in $I$, which send the boundary $\partial I=\{a, b\}$ into itself, and with leading coefficient of specified sign, compactified for $d$ even by the constant map with the same boundary behavior. (Compare Figures 5, 6.) For these families it has been proposed to generalize the monotonicity property of the topological entropy when $d=2$ to the connectedness of the topological entropy level sets or isentropes. Numerical computations in the case when $d=3$ have suggested the Connected Isentrope Conjecture according to which all isentropes are connected [M1]. (Compare the earlier report [DGMT]. In fact, for any $d$, one can ask the sharper question as to whether isentropes are contractible, or cellular.)

\footnotetext{
${ }^{2}$ In contrast with these older proofs, a recent proof by M. Tsujii does not depend on holomorphic dynamics, but does depend on complex analysis [Ts]. Notice that Tsuji indicates that his proof can be understood as a local version of the argument in [MTh].
} 
In the degree 3 case, the "bones", or sets of parameters such that one critical point is periodic with a specified order type, are no longer points, as when $d=2$, but are smooth curves. We conjectured [DGMT] that these bones cannot have any connected component which is a simple closed curve or "bone-loop". (It follows that every bone is a connected simple arc, except in a few exceptional cases with very low periods.) Furthermore, we sketched a proof of the following implications:

\section{Generic Hyperbolicity for Real Cubic Maps \\ $\Longrightarrow$ No Bone-Loops \\ $\Longrightarrow$ Isentropes are Connected.}

The generic hyperbolicity question remains open. However, C. Heckman [He] has been able to prove a weaker version which is enough to show that there are no bone-loops. The present paper will assume Heckman's result, and provide a more detailed exposition for the last implication. Thus, assuming that there are no bone loops, we will prove that isentropes, in either of the two families of real cubic maps, are indeed connected.

(In fact we will derive the slightly more precise statement that isentropes are cellular sets. However, even assuming generic hyperbolicity, we do not know whether isentropes are contractible. Compare $[\mathrm{FT}]$ which shows, for the family of analytic circle maps

$$
\theta \mapsto \theta+a+b \sin (\theta) \quad(\bmod 2 \pi),
$$

that the zero isentrope is non locally connected, with a "comb" structure.)

The paper is organized as follows. $§ 2$ contains a short discussion of kneading theory, generalized so as to allow maps with "plateaus" or intervals of constancy. $\S 3$ describes the parametrization of families of polynomial maps of the interval by their critical values. An alternate approach, due to Douady and Sentenac, is given in Appendix A. We discuss in $\S 4$ the main aspects of topological entropy that we need, in particular continuity properties and relations between topological entropy and kneading information. In $\S 5$, we describe families of continuous maps of the interval closely related to kneading theory, that we call stunted sawtooth maps: Corresponding to the family of degree $d$ polynomial maps with leading coefficient of specified sign, there is an essentially unique family of stunted sawtooth maps which mimic their behavior. Our basic hope is that most of the essential features of a family of polynomial maps of the interval are more or less faithfully mirrored in the corresponding stunted sawtooth family, where they are much easier to verify. As an example, the Generic Hyperbolicity Conjecture is easily verified for stunted sawtooth families. In $\S 6$, for each family of stunted sawtooth maps, we show that all isentropes are contractible, and therefore connected. This might be a step toward the Connected Isentrope Conjecture, or some more precise form of monotonicity property, for polynomial families of any degree. The rest of the paper is devoted to the proof that isentropes are connected for the two families of cubic maps. In order to use what we know for sawtooth maps in the study of cubic maps, we recall the definition of bones in $\S 7$, discuss their elementary properties in $\S 8$, and finish the proof in $\S 9$. 


\section{$\S 2$. Piecewise Monotone Maps and Kneading Theory.}

(Compare $[\mathrm{My}]$, [MSS], [MTh].) Let $I=[a, b]$ be a closed interval of real numbers. A map $f: I \rightarrow I$ will be called piecewise monotone if $I$ can be covered by finitely many closed intervals on which $f$ is monotone (but not necessarily strictly monotone ${ }^{3}$ ).

Let $\boldsymbol{\sigma}=\left(\sigma_{0}, \ldots, \sigma_{m}\right)$ be an alternating sequence of $m+1$ signs. That is, we assume that $\sigma_{j}=(-1)^{j} \sigma_{0}$ with $\sigma_{0}= \pm 1$. By an m-modal map of shape $\boldsymbol{\sigma}$ will be meant a piecewise monotone map $f: I \rightarrow I$ as above, together with a sequence of points

$$
a=c_{0}<c_{1}<\cdots<c_{m}<c_{m+1}=b
$$

in the interval $I=[a, b]$ satisfying the following condition. ${ }^{4}$ For each $0 \leq j \leq m$ the restriction of $f$ to the interval $c_{j} \leq x \leq c_{j+1}$ should be either monotone increasing or monotone decreasing according as $\sigma_{j}$ equals +1 or -1 . Thus the $c_{j}$ are uniquely determined by $f$ in the special case that $f$ is piecewise strictly monotone; but not in the general case when $c_{j}$ may belong to an interval of constancy. One also uses terms such as unimodal for an $m$-modal map with $m=1$, bimodal when $m=2$, and so on. We will use the notation

$$
(f, \mathbf{c}) \quad \text { with } \quad \mathbf{c}=\left(c_{1}, \ldots, c_{m}\right) \in I^{m}
$$

for an $m$-modal map when it is important to specify the precise choice of the $c_{i}$. We will call $c_{1}, \ldots, c_{m}$ the folding points of $f$, and their images $v_{i}=f\left(c_{i}\right)$ the folding values. In the case of a $C^{1}$-smooth function, note that the first derivative necessarily vanishes at our folding points, but may vanish at other points also. In the important case of $C^{2}$-smooth maps without critical inflection points, the $c_{i}$ are precisely the critical points of $f$, and their images $v_{i}$ are the critical values. However, we introduce a new name for these points in the general case in order to avoid confusion. In that special case, note that $\sigma_{j}$ can be identified with the sign of the first derivative $f^{\prime}(x)$ for $c_{j}<x<c_{j+1}$. (If the second derivative $f^{\prime \prime}\left(c_{j}\right)$ is non-zero, then $\sigma_{j}$ can also be identified with the sign of $f^{\prime \prime}\left(c_{j}\right)$ for $j=1, \ldots, m$.) When it is necessary to emphasize that some choice is involved (that is, when some of the $c_{i}$ lie in intervals of constancy) we may refer to the $c_{i}$ more explicitly as the designated folding points of the $m$-modal map $(f, \mathbf{c})$.

The $m$-tuple $\mathbf{v}=\left(v_{1}, \ldots, v_{m}\right) \in I^{m}$ will be called the folding value vector for $(f, \mathbf{c})$. Note that

$$
\begin{array}{lll}
v_{j} \leq v_{j+1} & \text { when } & \sigma_{j}=+1 \\
v_{j} \geq v_{j+1} & \text { when } & \sigma_{j}=-1
\end{array}
$$

for $0<j<m$. In fact, if we set $v_{0}=f(a), v_{m+1}=f(b)$, then this same inequality (2) will be true for $0 \leq j \leq m$. If strict equalities hold in (2) for $0 \leq j \leq m$, or equivalently

\footnotetext{
${ }^{3}$ We will use the term piecewise strictly monotone in the special case where $f$ has no intervals of constancy. However, it will be important to allow limiting cases where $f$ may have intervals of constancy. Because of this there is some choice of appropriate definitions for kneading theory (compare [BMT]), but the following will be convenient for our purposes.

${ }^{4}$ We will sometimes need to consider the more general case where

$$
a=c_{0} \leq c_{1} \leq \cdots \leq c_{m} \leq c_{m+1}=b .
$$

(Compare §3.) However, this is awkward for kneading theory, and we avoid it in this section.
} 
if $m$ is minimal, then $f$ will be called a strictly m-modal map, and $m+1$ will be called the lap number of $f$, denoted by $\ell(f)$. Thus $\ell(f)$ is the minimum number of intervals of monotonicity needed to cover the interval $I$.

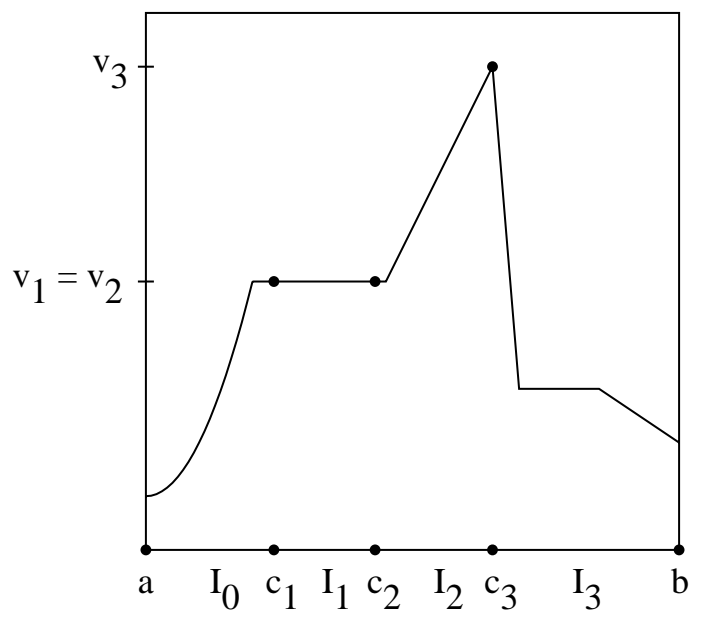

Figure 1. Graph of a 3-modal map of shape $(+-+-)$, with $v_{1}=v_{2}<v_{3}$. (Depending on the choice of designated folding points within the intervals of constancy, this same map could equally well be considered as strictly unimodal, or as $m$-modal for any odd value of $m$.)

Let $f: I \rightarrow I$ be an $m$-modal map of shape $\boldsymbol{\sigma}$. Partition the interval $I$ into disjoint subsets

$$
I=I_{0} \cup C_{1} \cup I_{1} \cup C_{2} \cup \cdots \cup C_{m} \cup I_{m}
$$

where $C_{j}$ is the set $\left\{c_{j}\right\}$ consisting of a single folding point of $f$, and where the open sets

$$
I_{0}=\left[c_{0}, c_{1}\right), I_{1}=\left(c_{1}, c_{2}\right), \cdots, I_{m}=\left(c_{m}, c_{m+1}\right]
$$

are the connected components of the complement $I \backslash\left(C_{1} \cup \cdots \cup C_{m}\right)$.

We will also think of the $C_{j}$ and $I_{j}$ as abstract symbols, forming the letters of an alphabet

$$
\mathfrak{A}=\mathfrak{A}(m)=\left\{I_{0}, C_{1}, I_{1}, C_{2}, \cdots, C_{m}, I_{m}\right\} .
$$

We order the $2 m+1$ symbols in $\mathfrak{A}(m)$ as they lie along the real line, so that

$$
I_{0}<C_{1}<I_{1}<C_{2}<\cdots<C_{m}<I_{m} .
$$

Each point $x \in I$ has a unique address $A(x) \in \mathfrak{A}$ defined by the condition that $x \in A(x)$. Note that $A(x) \leq A(y)$ whenever $x<y$.

Let $\mathfrak{A}^{\mathbb{N}}$ be the set of all infinite sequences $\left(A_{0}, A_{1}, \ldots\right)$ of symbols $A_{i} \in \mathfrak{A}$. Each point $x \in I$ has a well defined itinerary

$$
\mathcal{I}(x)=\left(A(x), A(f(x)), A\left(f^{\circ 2}(x)\right), \ldots\right) \in \mathfrak{A}^{\mathbb{N}} .
$$

Note that the map $f$ on the interval $I$ corresponds to the shift map

$$
\operatorname{shift}\left(A_{0}, A_{1}, \ldots\right)=\left(A_{1}, A_{2}, \ldots\right)
$$


on the space $\mathfrak{A}^{\mathbb{N}}$ of symbol sequences, in the sense that the following diagram is commutative

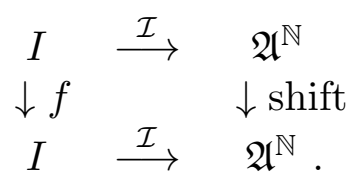

Some aspects of the structure of the orbit of $x$ reflect in an obvious way in the itinerary. For instance, if $x$ belongs to a periodic orbit, its itinerary must be periodic, and since the only periodic orbits of monotone maps have period 1 or 2 , the period of the itinerary of $x$ is either the period of the orbit or half of it. This periodic itinerary (or any shift of it) will be called the kneading type of the periodic orbit.

For each fixed shape $\boldsymbol{\sigma}$, we introduce a partial ordering of $\mathfrak{A}^{\mathbb{N}}$ as follows. Define the sign function $\epsilon: \mathfrak{A} \rightarrow\{-1,0,1\}$ by the formula $\epsilon\left(I_{j}\right)=\sigma_{j}$ and $\epsilon\left(C_{j}\right)=0$. (In the special case of a $C^{1}$-map whose derivative vanishes only at the folding points, note that $\epsilon(A(x))$ can be identified with the sign of the derivative $f^{\prime}(x)$.) By definition, $\left(A_{0}, A_{1}, \ldots\right)<\left(B_{0}, B_{1}, \ldots\right)$ if and only if there is a index $k \geq 0$ so that $A_{i}=B_{i}$ for $i<k$, but

$$
\begin{aligned}
& A_{k}<B_{k} \quad \text { if the product } \quad \epsilon\left(A_{0}\right) \epsilon\left(A_{1}\right) \cdots \epsilon\left(A_{k-1}\right) \quad \text { is equal to }+1 \\
& A_{k}>B_{k} \text { if this product equals }-1 \text {. }
\end{aligned}
$$

(If the product is zero, or in other words if some $A_{i}=B_{i}$ with $i<k$ is equal to one of the folding point sets $C_{1}, \ldots, C_{n}$, then the ordering is not defined. However, when comparing two different itineraries for the same map, this case occurs only when the two symbol sequences are identically equal.) It is not difficult to check that

$$
\mathcal{I}(x) \leq \mathcal{I}(y) \quad \text { whenever } \quad x \leq y .
$$

Note that there is a unique smallest element $\mathcal{I}_{\min }$ and a unique largest element $\mathcal{I}_{\max }$ in the space $\mathfrak{A}^{\mathbb{N}}$ with this ordering, so that $\mathcal{I}_{\min } \leq \mathcal{I} \leq \mathcal{I}_{\max }$ for all $\mathcal{I} \in \mathfrak{A}^{\mathbb{N}}$. For example:

$$
\mathcal{I}_{\text {min }}=\left\{\begin{array}{lll}
\left(I_{0}, I_{0}, I_{0}, I_{0}, \ldots\right) & \text { if } & \boldsymbol{\sigma}=(+\cdots \pm) \\
\left(I_{0}, I_{m}, I_{m}, I_{m}, \ldots\right) & \text { if } & \boldsymbol{\sigma}=(-\cdots+) \\
\left(I_{0}, I_{m}, I_{0}, I_{m}, \ldots\right) & \text { if } & \boldsymbol{\sigma}=(-\cdots-) .
\end{array}\right.
$$

Sometimes we will need to truncate the infinite sequences in $\mathfrak{A}^{\mathbb{N}}$ and consider the space $\mathfrak{A}^{k}$ of finite sequences of some fixed length $k$. There is a completely analogous ordering of such finite sequences.

The itineraries

$$
\mathcal{K}_{j}=\mathcal{I}\left(f\left(c_{j}\right)\right) \in \mathfrak{A}^{\mathbb{N}} .
$$

of the folding values $f\left(c_{j}\right)$ will play a special role, and will be called the kneading sequences of $f$. To each $m$-modal map $f$ there is associated a vector

$$
\mathbf{K}(f)=\left(\mathcal{K}_{1}, \ldots, \mathcal{K}_{m}\right) \in\left(\mathfrak{A}^{\mathbb{N}}\right)^{m}
$$

of kneading sequences. This vector of kneading sequences, together with the shape $\boldsymbol{\sigma}$, will be called the kneading data for the piecewise monotone map $f$. 
The choice of kneading data imposes sharp restrictions on which itineraries can actually occur. Suppose that $\mathcal{I}(x)=\left(A_{0}, A_{1}, \ldots\right)$. Evidently:

Compatibility Condition 1. If some symbol $A_{k}$ is equal to $C_{j}$, then the sequence $\left(A_{k+1}\right.$, $\left.A_{k+2}, \ldots\right)$ which follows it must be equal to the kneading sequence $\mathcal{K}_{j}$.

In view of this condition, it is often convenient to terminate the sequence $\left(A_{0}, A_{1}, \ldots\right)$ at the first $C_{j}$ which occurs in it, since the subsequent symbols give no further information.

Compatibility Condition 2. If $A_{k}=I_{j-1}$ or $A_{k}=I_{j}$, then the sequence which follows must satisfy either

$$
\left(A_{k+1}, A_{k+2}, \ldots\right) \leq \mathcal{K}_{j}
$$

or

$$
\left(A_{k+1}, A_{k+2}, \ldots\right) \geq \mathcal{K}_{j}
$$

according as $\sigma_{j}=-1$ (so that $c_{j}$ is a local maximum point) or $\sigma_{j}=+1$ (so that $c_{j}$ is a local minimum point).

Definition. The symbol sequence $\left(A_{0}, A_{1}, \cdots\right) \in \mathfrak{A}^{\mathbb{N}}$ will be called admissible for the kneading data $\left(\mathcal{K}_{1}, \ldots, \mathcal{K}_{m}\right)$ if it satisfies these two compatibility conditions. (This terminology will be justified in 5.2.)

Remark. Evidently the orbit of the folding point $c_{j}$ is periodic if and only if the itinerary $\mathcal{I}\left(c_{j}\right)=\left(C_{j}, \mathcal{K}_{j}\right)$ is periodic. Using Condition 1 , a completely equivalent condition is that the kneading sequence $\mathcal{K}_{j}$ contains the symbol $C_{j}$ as one of its entries.

Example 2.1. The map $f(x)=(4 x-1)^{2}(1-x)$ of shape $(-+-)$ on the unit interval has periodic kneading sequences $\mathcal{K}_{1}=\overline{I_{0} I_{2}}$ and $\mathcal{K}_{2}=\overline{I_{2} I_{0}}$ (where the overline indicates a sequence which is to be repeated infinitely often), yet the critical points themselves are not periodic. In fact $f$ maps both critical points $1 / 4$ and $3 / 4$ to the boundary period two orbit $\{0,1\}$. On the other hand, for the map $f(x)=1-3 x^{2}+2 x^{3}$ of shape $(+-+)$ on the interval bounded by $(1 \pm \sqrt{5}) / 2$, the critical points $0 \leftrightarrow 1$ are periodic, and hence their itineraries $\mathcal{I}(0)=\overline{C_{1} C_{2}}$ and $\mathcal{I}(1)=\overline{C_{2} C_{1}}$ are also periodic.

In general, one cannot hope that the set of all itineraries for a given map will be completely determined by its kneading data. The first problem is that the kneading data does not usually determine the itineraries of the two endpoints $a, b \in \partial I$. Yet every itinerary $\mathcal{I}(x)$ which actually occurs must certainly satisfy

$$
\mathcal{I}(a) \leq \mathcal{I}(x) \leq \mathcal{I}(b) .
$$

If these two boundary itineraries have been specified, then setting $\mathcal{K}_{0}=\mathcal{I}(f(a))$ and $\mathcal{K}_{m+1}=\mathcal{I}(f(b))$ we can introduce the following slightly sharper version of Condition 2 for an itinerary $\mathcal{I}(x)=\left(A_{0}, A_{1}, \ldots\right)$ :

Compatibility Condition $2^{\sharp}$. If $A_{k}=I_{j}$, then

$$
\begin{array}{lll}
\mathcal{K}_{j} \leq\left(A_{k+1}, A_{k+2}, \ldots\right) \leq \mathcal{K}_{j+1} & \text { whenever } & \sigma_{j}=+1 \\
\mathcal{K}_{j} \geq\left(A_{k+1}, A_{k+2}, \ldots\right) \geq \mathcal{K}_{j+1} & \text { whenever } & \sigma_{j}=-1 .
\end{array}
$$


However, it is still not true that every sequence satisfying Conditions 1 and $2^{\sharp}$ necessarily occurs as itinerary for the given $m$-modal map. The following helps to indicate the more serious difficulties.

Example 2.2. Consider the following three families of unimodal maps of shape $(+-)$ on the unit interval, with folding point $1 / 2$, all parametrized by the folding value $v$,

$$
\begin{array}{lll}
\text { the tent family: } & T_{v}(x)=2 v \operatorname{Min}(x, 1-x), \\
\text { the quadratic family: } & Q_{v}(x)=4 v x(1-x), \\
\text { and the family: } & \left.S_{v}(x)=\operatorname{Min}(2 x, v, 2-2 x) \quad \text { (compare } \S 5\right) .
\end{array}
$$

In each case there is a unique parameter value $v$ so that the orbit of the folding point has period 3 , necessarily with kneading sequence $\mathcal{K}_{1}=\overline{I_{1} I_{0} C_{1}}$. (The corresponding parameter values are respectively

$$
v=(1+\sqrt{5}) / 4=0.80901 \cdots, \quad v^{\prime}=0.95796 \cdots, \quad \text { and } \quad v^{\prime \prime}=7 / 8=0.875
$$

in the three cases.) However, the itineraries which can actually occur for these three maps are different. For the tent case, the orbit of the folding point is the only period 3 orbit. (The graph of $T_{v}^{\circ 3}$ touches the diagonal without crossing it at the three points of this orbit.) In the other two cases the corresponding graph definitely crosses the diagonal and must cross back, so there is a period 3 point close to the folding point with itinerary $\overline{I_{1} I_{1} I_{0}}$. In the third case, the corresponding graph is horizontal near the folding point, so that there are also infinitely many nearby points with an itinerary $\mathcal{I}(x)$ equal to

$$
I_{0} \mathcal{K}_{1}=I_{0} \overline{I_{1} I_{0} C_{1}} \quad \text { or } \quad I_{1} \mathcal{K}_{1}=I_{1} \overline{I_{1} I_{0} C_{1}} .
$$

Evidently such itineraries can occur only if the map is constant on the entire interval between $x$ and the folding point. Hence they can never occur for maps like $T_{v}$ or $Q_{v^{\prime}}$ which are piecewise strictly monotone.

Thus the compatibility conditions are not sufficient to guarantee the existence of itineraries, or even of finite truncations of itineraries which contain folding point symbols. However, the next lemma provides a useful existence statement by working only with finite sequences containing no folding point symbols. Note that the analogues of Conditions 1,2 , and $2^{\sharp}$ for finite sequences in $\mathfrak{A}^{k}$ make perfect sense. In particular, the concept of "admissibility" for finite sequences also makes sense. A finite or infinite symbol sequence $\left\{A_{i}\right\}$ will be called acritical if the $A_{i}$ all belong to the smaller alphabet

$$
\mathfrak{A}_{0}=\mathfrak{A}_{0}(m)=\left\{I_{0}, I_{1}, \ldots, I_{m}\right\} \subset \mathfrak{A}(m),
$$

with no folding point symbols.

Lemma 2.3. Let $f$ be an m-modal map, and let $\left(I_{\alpha_{0}}, I_{\alpha_{1}}, \ldots, I_{\alpha_{k}}\right)$ be a finite sequence of intervals $I_{\alpha_{j}} \in \mathfrak{A}_{0}(m)$. There exists an orbit

$$
x_{0} \mapsto x_{1} \mapsto \cdots \mapsto x_{k} \mapsto \cdots
$$

with $x_{i} \in I_{\alpha_{i}}$ for all $i \leq k$ if and only if this sequence satisfies Condition $2^{\sharp}$, modified so as to apply to sequences of finite length.

Proof. If there exists such an orbit, then clearly the sequence $\left(I_{\alpha_{0}}, \ldots, I_{\alpha_{k}}\right)$ must 
satisfy the suitably modified Condition $2^{\sharp}$. Conversely, we will prove by induction on $k$ that every sequence $\left(I_{\alpha_{0}}, I_{\alpha_{1}}, \ldots, I_{\alpha_{k}}\right)$ which satisfies this condition can be realized by an orbit of $f$. This statement is certainly true when $k=0$. Suppose then that it is known for all sequences of shorter length. Suppose also, to fix our ideas, that $\sigma_{\alpha_{0}}=+1$, so that

$$
\mathcal{K}_{\alpha_{0}}^{(k)} \leq\left(I_{\alpha_{1}}, I_{\alpha_{2}}, \ldots, I_{\alpha_{k}}\right) \leq \mathcal{K}_{\alpha_{0}+1}^{(k)}
$$

by Condition $2^{\sharp}$. (Here the superscript $(k)$ indicates truncation to length $k$.) By the induction hypothesis, there exists a point $x_{1} \in I$ with itinerary

$$
\left(I_{\alpha_{1}}, I_{\alpha_{2}}, \ldots, I_{\alpha_{k}}, \ldots\right) \text {. }
$$

The proof will now be divided into two cases according as strict inequalities do or do not hold in (4). If strict inequalities hold, then it follows that $v_{\alpha_{0}}<x_{1}<v_{\alpha_{0}+1}$. It then follows from the intermediate value theorem that there exists at least one point $x_{0} \in I_{\alpha_{0}}$ with $f\left(x_{0}\right)=x_{1}$, so that $x_{0}$ has the required itinerary. On the other hand, if for example

$$
\mathcal{K}_{\alpha_{0}}^{(k)}=\left(I_{\alpha_{1}}, I_{\alpha_{2}}, \ldots, I_{\alpha_{k}}\right),
$$

then the point $x_{0}=c_{\alpha_{0}}+\epsilon$ with $\epsilon>0$ sufficiently small will have the required itinerary, since the $I_{\alpha_{j}}$ are open subsets of $I$. The remaining cases are completely analogous.

It is often practical to first consider maps whose boundary behavior is specified in the simplest way. Suppose that $f$ maps the interval $I=[a, b]$ into itself. Again choose some fixed shape $\boldsymbol{\sigma}$.

Definition. The $m$-modal map $f: I \rightarrow I$ will be called boundary anchored for the shape $\boldsymbol{\sigma}$ if $f$ maps the boundary $\partial I=\{a, b\}$ into itself by the rule

$$
f(a)=\left\{\begin{array}{lll}
a & \text { if } & \sigma_{0}=+ \\
b & \text { if } & \sigma_{0}=-,
\end{array} \quad f(b)=\left\{\begin{array}{lll}
b & \text { if } & \sigma_{m}=+ \\
a & \text { if } & \sigma_{m}=-.
\end{array}\right.\right.
$$

In the sequel, we will nearly always work with boundary anchored maps, so that the distinction between Conditions 2 and $2^{\sharp}$ will disappear.
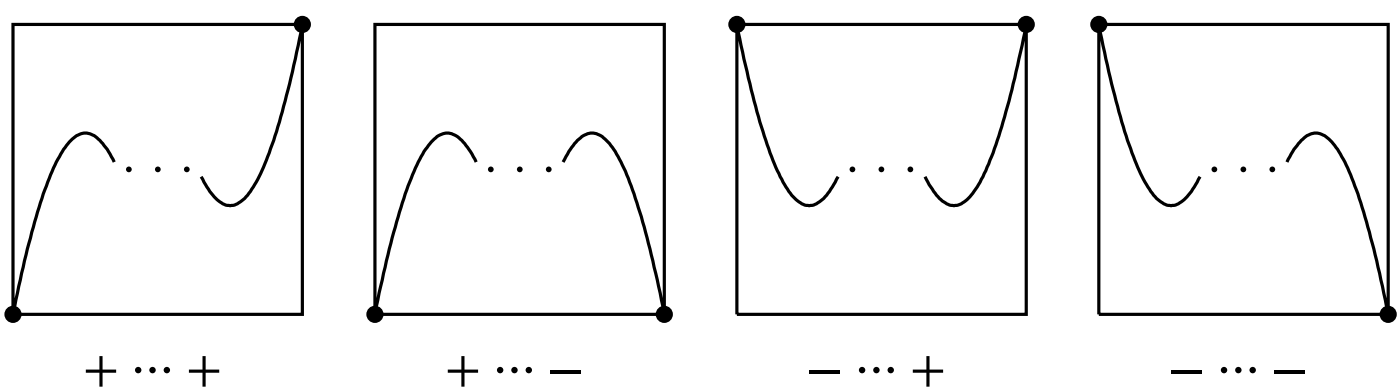

Figure 2. Boundary anchored maps: graphs illustrating the four possible cases. These maps are $m$-modal with $m$ odd for the middle pictures, and with $m$ even for the end pictures.

For a boundary anchored map, note that the itinerary $\mathcal{I}(a)$ is precisely equal to $\mathcal{I}_{\min }$ and that $\mathcal{I}(b)=\mathcal{I}_{\max }$. It follows that the sequences $\mathcal{K}_{0}=\mathcal{I}(f(a))$ and $\mathcal{K}_{m+1}=\mathcal{I}(f(b))$ 
are determined by the shape, and that each one is equal to either $\mathcal{I}_{\min }$ or $\mathcal{I}_{\max }$. Lemma 2.3 has the following consequence. (Compare 4.5 below.)

Corollary 2.4. Let $f$ be a boundary anchored m-modal map. Then a finite acritical sequence $\left(I_{\alpha_{0}}, \ldots, I_{\alpha_{k}}\right)$ is actually realized by some orbit $x_{0} \mapsto \cdots \mapsto x_{k}$ if and only if it is admissible. Hence, for any $k$, the set of all such sequences in $\mathfrak{A}_{0}^{k}$ is determined by the kneading data for $f$.

The kneading sequences $\mathcal{K}_{j}$ are themselves itineraries, and hence must satisfy these two compatibility conditions. Evidently they must also satisfy the following.

Compatibility Condition 3 (for kneading data).

$$
\begin{array}{lll}
\mathcal{K}_{j} \leq \mathcal{K}_{j+1} & \text { if } & \sigma_{j}=+1, \\
\mathcal{K}_{j} \geq \mathcal{K}_{j+1} & \text { if } & \sigma_{j}=-1 .
\end{array}
$$

Definition. The kneading data $\mathbf{K}=\left(\mathcal{K}_{1}, \ldots, \mathcal{K}_{m}\right)$ will be called admissible for the specified shape $\boldsymbol{\sigma}$ if it satisfies these three compatibility conditions.

As we shall see in 5.2 , the three compatibility conditions characterize the $m$-tuples of symbolic sequences in $\mathfrak{A}(m)^{\mathbb{N}}$ that can actually occur as kneading data for some $m$-modal map. However, if we restrict to polynomial (or analytic) maps, then further restrictions are needed. (Compare [MaT1, p.179], as well as 2.2 and Appendix B, Example 4.)

\section{$\S 3$. Parametrization of polynomials.}

First consider a polynomial map $f: \mathbb{R} \rightarrow \mathbb{R}$ of degree $m+1$ with distinct real critical points

$$
c_{1}<c_{2}<\cdots<c_{m} .
$$

As in the previous section, we can form the critical value vector (= folding value vector) $\mathbf{v}=\left(v_{1}, \ldots, v_{m}\right) \in \mathbb{R}^{m}$, where $v_{i}=f\left(c_{i}\right)$. Setting $\sigma_{m}$ equal to the sign of the leading coefficient, or equivalently the sign of the $(m+1)$-st derivative, and setting $\sigma_{i}=(-1)^{m-i} \sigma_{m}$, note the following strict form of (2):

$$
\sigma_{i}\left(v_{i+1}-v_{i}\right)>0 \quad \text { for } \quad 1 \leq i<m .
$$

Again we will refer to $\boldsymbol{\sigma}=\left(\sigma_{0}, \ldots, \sigma_{m}\right)$ as the shape. We will first prove the following.

Lemma 3.1. Given any $m$-tuple $\left(v_{1}, \ldots, v_{m}\right) \in \mathbb{R}^{m}$ satisfying the inequalities $\left(2^{\prime}\right)$, there exists a polynomial $f$ of degree $m+1$ with these critical values, listed in order of the corresponding critical points as above. Furthermore $f$ is unique up to precomposition with a positive affine transformation

$$
f(x) \mapsto f(p x+q) \quad \text { with } \quad p>0 .
$$

Proof of 3.1. We will give a proof based on complex analysis. Alternatively, a purely real proof may be extracted from [MvS2, p. 120.], and a different real proof, due to Douady and Sentenac, is provided in Appendix A. 
To begin the construction, start with $m+1$ copies of the Riemann sphere $\hat{\mathbb{C}}$, labeled as $\{k\} \times \hat{\mathbb{C}}$ for $0 \leq k \leq m$. Slit the first two copies $\{0\} \times \hat{\mathbb{C}}$ and $\{1\} \times \hat{\mathbb{C}}$ along the real axis from $v_{1}$ to $\pm \infty$, taking the plus sign or the minus sign according as the sign $\sigma_{0}$ is +1 or -1 . That is, if $\sigma_{0}=+1$ so that $v_{1}$ is a local maximum, we remove the open interval consisting of real $z$ with $v_{1}<z<+\infty$, while if $\sigma_{0}=-1$ so that $v_{1}$ is a local minimum we remove the open interval $-\infty<z<v_{1}$. Similarly, for each $1 \leq k \leq m$ we slit both $\{k-1\} \times \hat{\mathbb{C}}$ and $\{k\} \times \hat{\mathbb{C}}$ from $v_{k}$ to $\pm \infty$, now choosing the sign according as $v_{k}$ is a local maximum or minimum. The hypothesis $\left(2^{\prime}\right)$ guarantees that these various slits can never meet.
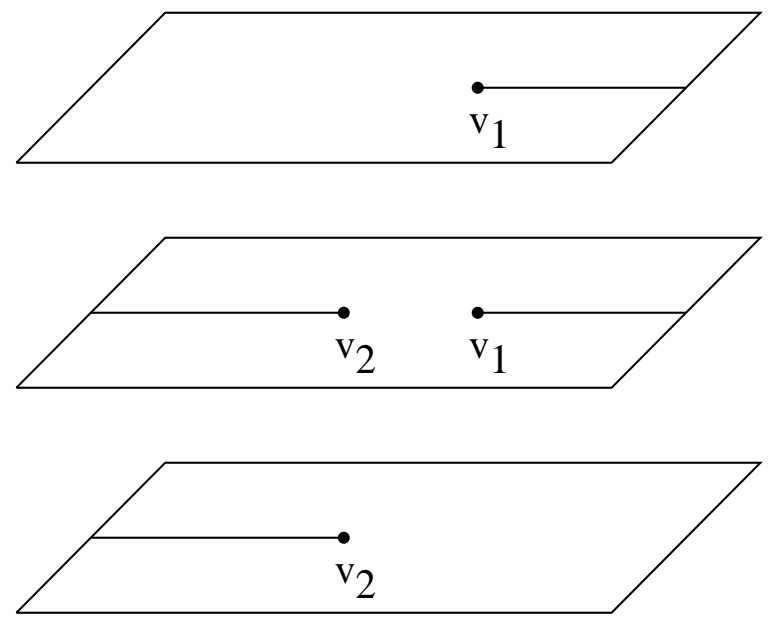

Figure 3. Three copies of the complex numbers, slit for the construction of a bimodal map of shape $(+-+)$, with critical values $v_{1}>v_{2}$.

Now, for each $1 \leq k \leq m$, sew together the pair of corresponding slits in $\{k-1\} \times \hat{\mathbb{C}}$ and $\{k\} \times \hat{\mathbb{C}}$ so that the top edge of either one is matched with the bottom edge of its mate. The result will be a compact simply connected Riemann surface $S$. (Note that there is a canonical way of assigning a conformal structure to this surface $S$, even at the $m+1$ ramification points.) Furthermore, the natural projection maps $(k, z) \mapsto z$ from $\{k\} \times \hat{\mathbb{C}}$ to $\hat{\mathbb{C}}$ fit together to yield a holomorphic map $\eta: S \rightarrow \hat{\mathbb{C}}$ of degree $m+1$, with the $v_{k}$ and the point at infinity as critical values. By the Uniformization Theorem, $S$ must be conformally isomorphic to the standard Riemann sphere, under some isomorphism $u: \hat{\mathbb{C}} \rightarrow S$. (See for example $[\mathrm{FK}]$ or $[\mathrm{Be}]$.$) If we choose the isomorphism u: \hat{\mathbb{C}} \rightarrow S$ in such a way that the points at infinity correspond, then the composition $\eta \circ u: \hat{\mathbb{C}} \rightarrow \hat{\mathbb{C}}$ is clearly a polynomial map with the required critical values.

To recover the real mapping, note that the complex conjugation operations on the various copies $\{k\} \times \hat{\mathbb{C}}$ fit together to yield an anti-holomorphic involution of $S$ whose fixed point set $F$ is the union of the $m+1$ real axes, with the various slits removed. There is a preferred ordering of $F \backslash\{\infty\}$ so that its intersections with the various $\{k\} \times \hat{\mathbb{C}}$ occur in the order of increasing $k$. Now choose the conformal isomorphism $u: \hat{\mathbb{C}} \rightarrow S$ so that 
$\mathbb{R} \subset \hat{\mathbb{C}}$ corresponds to $F \backslash\{\infty\}$, preserving orientation. In this way, we obtain a real polynomial map $\left.\eta \circ u\right|_{\mathbb{R}}$, with the specified critical values occurring in the specified order. Evidently the conformal isomorphism $u$, with these restrictions, is unique up to an affine change of coordinates

$$
u(z) \mapsto u(p z+q)
$$

with $p, q \in \mathbb{R}$ and $p>0$.

In practice, we are interested in polynomial maps which carry some closed interval $I=[a, b] \subset \mathbb{R}$ into itself, and which are boundary anchored, so that $\partial I=\{a, b\}$ maps into itself. Let us fix the shape $\boldsymbol{\sigma}$. It is sometimes convenient to set

$$
v_{0}=v_{0}(\boldsymbol{\sigma})=f(a), \quad v_{m+1}=v_{m+1}(\boldsymbol{\sigma})=f(b),
$$

where now $f(a)$ and $f(b)$ are to be defined by formula (5). Thus a map $g$ is boundary anchored for the shape $\boldsymbol{\sigma}$ if and only if it satisfies $g(a)=v_{0}$ and $g(b)=v_{m+1}$. Note that the inequality $\left(2^{\prime}\right)$ can be sharpened to include $v_{0}$ and $v_{m+1}$ :

$$
\sigma_{i}\left(v_{i+1}-v_{i}\right)>0 \quad \text { for } \quad 0 \leq i \leq m
$$

Theorem 3.2. Given an $m$-tuple $\left(v_{1}, \ldots, v_{m}\right) \in I^{m}$ satisfying the inequalities $\left(2^{\prime \prime}\right)$, there exists one and only one boundary anchored polynomial map $g: I \rightarrow I$ of degree $m+1$ which has distinct critical points

$$
a<c_{1}<\cdots<c_{m}<b \text {, }
$$

with $g\left(c_{k}\right)=v_{k}$.

Proof of 3.2. Let $f: \mathbb{R} \rightarrow \mathbb{R}$ be the polynomial whose existence is promised by 3.1 . Then we claim that there are unique real numbers $p>0$ and $q$ so that the polynomial $g(x)=f(p x+q)$ satisfies the additional conditions that $g(\partial I) \subset \partial I$. It is then easy to check that $g(I) \subset I$.

Without loss of generality, we may assume that $I$ is the unit interval $[0,1]$. Suppose first that $\boldsymbol{\sigma}$ has the form $(+\cdots-)$. Then $f\left(c_{1}\right)=v_{1}$ is a local maximum point. Evidently $f$ maps the closed interval $\left[-\infty, c_{1}\right]$ homeomorphically onto the closed interval $\left[-\infty, v_{1}\right]$. Since $v_{1}>a=0$, there is a unique point $-\infty<q<c_{1}$ with $f(q)=0$. Similarly, $f$ maps the closed interval $\left[c_{m},+\infty\right]$ homeomorphically onto $\left[-\infty, v_{m}\right]$, reversing orientation. Since $v_{m}>a=0$, there is a unique $p+q$ with $c_{m}<p+q<+\infty$ and $f(p+q)=0$. The map $g(x)=f(p x+q)$ will then have the required properties.

The proof for the other three possible shapes is similar. Details will be left to the reader.

Thus far, we have assumed that our polynomials have distinct critical points, however it is often convenient to relax this condition in order to obtain a compact parameter space. First consider polynomials $f(x)$ with derivative

$$
f^{\prime}(x)=\kappa\left(x-c_{1}\right) \cdots\left(x-c_{m}\right) \quad \text { where } \quad a \leq c_{1} \leq \cdots \leq c_{m} \leq b
$$

for some real constant $\kappa \neq 0$. The corresponding critical value vector $\left(v_{1}, \ldots, v_{m}\right)$ then 
satisfies the weaker inequalities (2):

$$
\left(v_{i+1}-v_{i}\right) \sigma_{i} \geq 0 \quad \text { for } \quad 1 \leq i<m,
$$

where $\sigma_{i}=(-1)^{m-i} \operatorname{sgn}(\kappa)$. Just as in Lemma 3.1, the polynomial $f$ is uniquely determined, up to precomposition with a positive affine transformation, by its critical value vector, which is required to satisfy only (2). Either the proof above or the proof in Appendix A can be adapted to show this. (Compare the Addendum to Appendix A.) Details will be omitted.

There is a corresponding generalization of 3.2. However, when $m$ is odd, we must now allow for the case of a constant function with $f(x)$ identically equal to $a$ or $b$. In this limiting case, note that the critical value vector $v_{1}=\cdots=v_{m}$ is still well defined, although we can no longer distinguish $m$ critical points. With this understanding, we have the following.

Theorem 3.3. Given a specified shape $\boldsymbol{\sigma}$, and given $\left(v_{1}, \ldots, v_{m}\right) \in I^{m}$ satisfying (2), there is one and only one boundary anchored polynomial map $f: I \rightarrow I$ of degree $m+1$ having $\left(v_{1}, \ldots, v_{m}\right)$ as critical value vector.

The proof is quite similar to the proof of 3.2 .
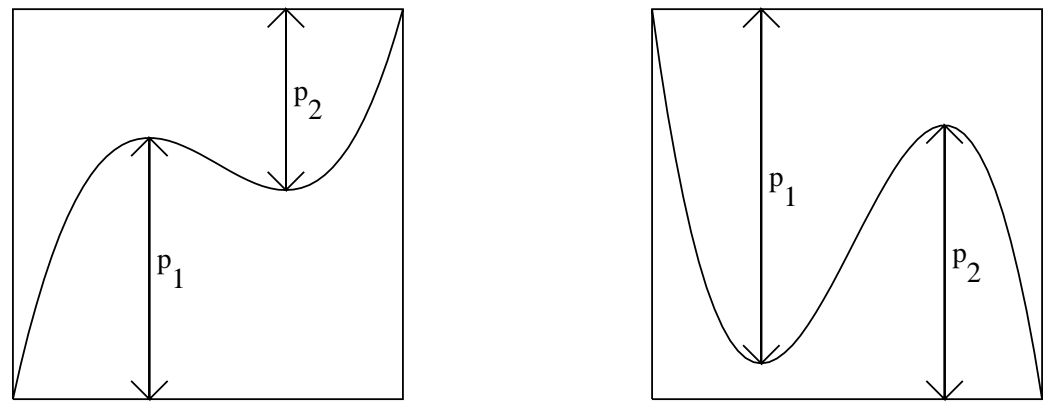

Figure 4. The parameters $p_{1}$ and $p_{2}$ for a cubic map of shape +-+ on the left, and -+- on the right, on an interval of length 1.

It would be quite natural to parametrize the set of all boundary anchored polynomial maps $f: I \rightarrow I$ of shape $\boldsymbol{\sigma}$ by the polyhedron consisting of all vectors $\mathbf{v}=\left(v_{1}, \ldots, v_{m}\right) \in$ $I^{m}$ satisfying the inequalities (2) for the shape $\boldsymbol{\sigma}$. However, we will rather work with a slightly different but affinely equivalent polyhedron which is independent of the shape $\boldsymbol{\sigma}$, and is invariant under affine reparametrization of the interval $I$ (as used in renormalization theory). As a first attempt in this direction, note that our interval $I$ is the union of nonoverlapping subintervals $I_{j}$, which map onto intervals $f\left(I_{j}\right) \subset I$. Let

$$
\Delta_{j}=\frac{\operatorname{length}\left(f\left(I_{j}\right)\right)}{\operatorname{length}(I)} \in[0,1]
$$

be the relative length of this image interval.

Since our maps are boundary anchored, these invariants $\Delta_{0}, \ldots, \Delta_{m}$ are not independent. (Their alternating sum is constant, and various inequalities are needed to guarantee 
that $f(I) \subset I$.) However, we can obtain a set of invariants which are independent and more manageable by setting

$$
\Delta_{j}=p_{j}+p_{j+1}-1 \quad \text { for } \quad 0<j<m
$$

with

$$
\Delta_{0}=p_{1}, \quad \Delta_{m}=p_{m} .
$$

(Note that the "normalized total variation" $\sum \Delta_{i}$ of our $m$-modal map is equal to a constant plus $2\left(p_{1}+\cdots+p_{m}\right)$.)

If $I$ is the interval $[a, b]$, then we can write

$$
p_{i}=\left\{\begin{array}{lll}
\left(v_{i}-a\right) /(b-a) & \text { if } & \sigma_{i}=+1 \\
\left(b-v_{i}\right) /(b-a) & \text { if } & \sigma_{1}=-1 .
\end{array}\right.
$$

(Compare Figures 4,9.) In particular, in the special case of the unit interval $I=[0,1]$ this formula simplifies to

$$
p_{i}=\left\{\begin{array}{lll}
v_{i} & \text { if } & \sigma_{i}=+1 \\
1-v_{i} & \text { if } & \sigma_{1}=-1
\end{array}\right.
$$

It is easy to check that the parameters $p_{1}, \ldots, p_{m} \in[0,1]$ satisfy only the relations

$$
p_{i}+p_{i+1} \geq 1 \text {. }
$$

It may seem that an increase in any $p_{i}$ should lead to more complex dynamical behavior, but this is not quite true for the cubic family. (Compare Figure 8.) However, in 5.6 we will see that the analogous statement for the family of "stunted sawtooth" maps is true.
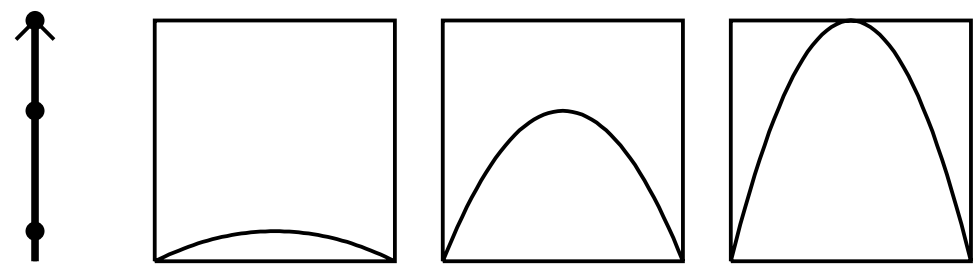

Figure 5. Picture of the polyhedron $P^{1}$ on the left, followed by the graphs of the quadratic maps of shape +- corresponding to three representative points of $P^{1}$.

As parameter space for boundary anchored polynomial maps of degree $m+1$ and shape $\boldsymbol{\sigma}$ (or indeed for any family of maps which can be parametrized by their folding value vectors) we take the polyhedron $P^{m}$ consisting of all vectors $\mathbf{p}=\left(p_{1}, \ldots, p_{m}\right) \in[0,1]^{m}$ which satisfy the inequalities (7). This is a convex polyhedral subset of the $m$-dimensional unit cube. More exactly, $P^{m}$ can be described as the convex hull of the set of vectors $\left(p_{1}, p_{2}, \ldots, p_{m}\right)$ with $p_{i} \in\{0,1\}$, such that there is no consecutive pair of 0 's. From this characterization, we get:

Proposition 3.4. The polyhedron $P^{m}$ has $F_{m+1}$ vertices, where $F_{i}$ stands for the $i^{\text {th }}$ Fibonacci number.

Proof of 3.4. Recall that the sequence of Fibonacci numbers is defined by $F_{n+2}=$ $F_{n}+F_{n+1}$ for $n>0$, with $F_{1}=F_{2}=1$. Notice first that $P^{2}$ is a segment with $F_{1}=1$ 

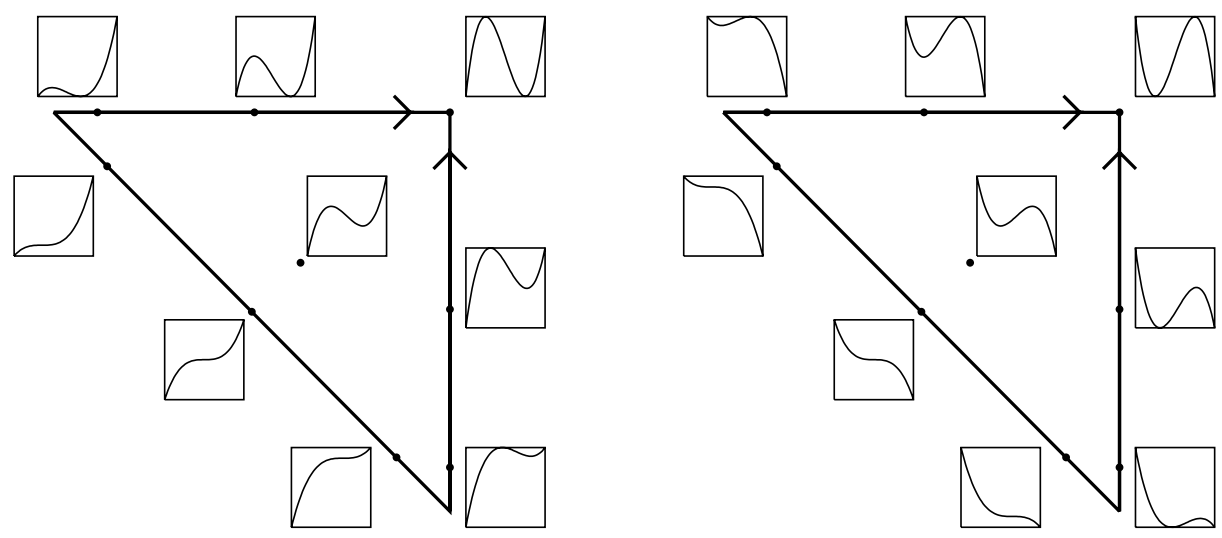

Figure 6. The triangle $P^{2}$, showing cubic maps of shape +-+ on the left, and cubic maps of shape -+- on the right, for nine representative points of $P^{2}$. Arrows point in the direction of the $p_{1}$ and $p_{2}$ axes.

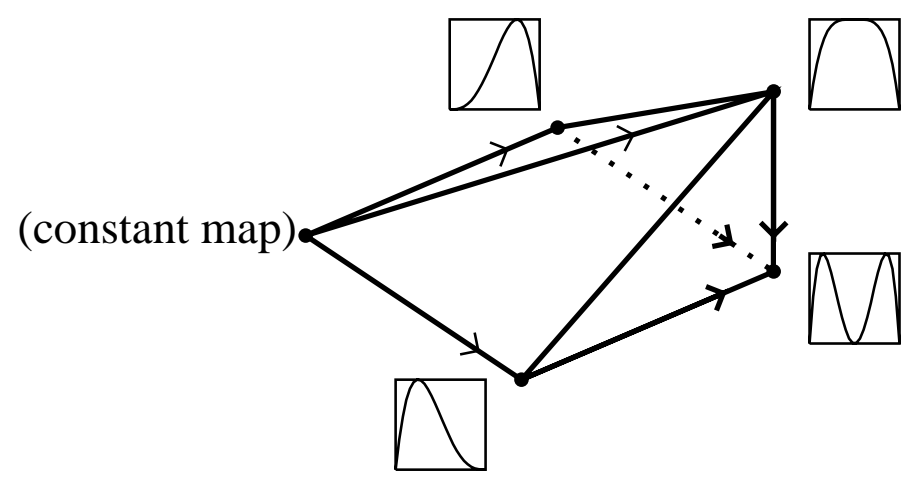

Figure 7. The polyhedron $P^{3}$ together with graphs of the 4-th degree maps of shape +-+- corresponding to its vertices. Arrows point in the direction of increasing entropy, with heavy arrows parallel to the $p_{j}$ axes.

vertex labeled 0 and $F_{2}=1$ vertex labeled 1 . Next, if $P^{m}$ has $F_{m-1}$ vertices whose label terminates with 0 and $F_{m}$ vertices whose label terminates with 1 , the "not two consecutive zeros" rule implies that $P^{m+1}$ has $F_{m}$ vertices whose label terminates by 0 and $F_{m-1}+F_{m}=F_{m+1}$ vertices whose label terminates with 1 .

While the abstract polyhedron $P^{m}$ is completely determined by $m$, the way it is partitioned according to dynamical properties depends of course on the particular family of maps it parameterizes. Consider some family of $m$-modal maps parametrized by $P^{m}$ : we will write $\mathbf{p}=\mathbf{p}(\mathbf{v})$ (which can be inverted to $\mathbf{v}=\mathbf{v}(\mathbf{p})$ ) so that, for any chosen shape, $f_{\mathbf{p}}$ is the boundary anchored map on $I$ in that family, which corresponds to the parameter vector $\mathbf{p}$ and to the folding value vector $\mathbf{v}$. For example, in the polynomial case, the map $f_{(1, \ldots, 1)}$ corresponding to the vector $\mathbf{p}=(1,1, \ldots, 1)$ is, up to sign, a Chebyshev polynomial: it is the unique boundary anchored polynomial map of shape $\boldsymbol{\sigma}$ with maximal topological entropy. 
Note: When $m$ is odd, the two possible shapes for an $m$-modal map are not really different, since a map of shape $(+-\cdots+-)$ with parameters $\left(p_{1}, \ldots, p_{m}\right)$ is topologically conjugate, under an orientation reversing reflection of its interval, to a map of shape $(-+\cdots-+)$ with parameters $\left(p_{m}, \ldots, p_{1}\right)$. However, when $m$ is even the two shapes are dynamically essentially different. (Even the dynamical behavior of $f$ on the boundary of $I$ is enough to distinguish the two.)

\section{§. Topological Entropy and Periodic Orbits.}

A particularly useful measure of the dynamic "complexity" of a continuous self-map is provided by the topological entropy $h$ which was defined in [AKM] as an invariant of topological conjugacy. The definition requires some notations. Let $X$ be a non-vacuous compact space. By a cover $\mathfrak{C}$ we mean simply a collection of subsets with union $X$. The "covering number" $\mathbf{n}(\mathfrak{C})$ is defined to be the smallest cardinality of a subcollection of $\mathfrak{C}$ with union $X$. Given a continuous map $f: X \rightarrow X$ and $k \geq 1$, define $\mathfrak{C}_{f}^{k}$ to be the cover consisting of all intersections of the form

$$
C_{0} \cap f^{-1} C_{1} \cap \cdots \cap f^{1-k} C_{k-1}
$$

where each $C_{i}$ is a set belonging to the collection $\mathfrak{C}$. (Here $f^{-i}(C)$ is the set of all $x \in X$ with $f^{\circ i}(x) \in C$.) Using the inequality $\mathbf{n}\left(\mathfrak{C}_{f}^{k+\ell}\right) \leq \mathbf{n}\left(\mathfrak{C}_{f}^{k}\right) \cdot \mathbf{n}\left(\mathfrak{C}_{f}^{\ell}\right)$, one can check that the limit

$$
h(f, \mathfrak{C})=\lim _{k \rightarrow \infty} \frac{1}{k} \log \mathbf{n}\left(\mathfrak{C}_{f}^{k}\right)
$$

exists, with $0 \leq h(f, \mathfrak{C}) \leq \infty$. Furthermore, this limit is equal to the infimum

$$
\inf _{k>0} \frac{1}{k} \log \mathbf{n}\left(\mathfrak{C}_{f}^{k}\right) \text {. }
$$

In particular, $h(f, \mathfrak{C}) \leq \mathbf{n}(\mathfrak{C})$, so if $\mathbf{n}(\mathfrak{C})$ is finite (for example if $\mathfrak{C}$ is a covering by finitely many sets, or if $\mathfrak{C}$ is a covering of the compact space $X$ by open subsets), then it follows that $h(f, \mathfrak{C})$ must also be finite.

The topological entropy of $f$ is defined to be the the supremum of $h(f, \mathfrak{C})$ over all open covers of $X$. (This may be infinite, even if each $h(f, \mathfrak{C})$ is finite, since covers by smaller sets may yield larger values of $h(f, \mathfrak{C})$.)

In the case of maps of the interval, combining theorems of Misiurewicz and Yomdin, we have the following. Let $C^{\infty}(I, I)$ be the space of $C^{\infty}$ maps from a closed interval $I$ to itself, with the $C^{\infty}$ topology.

Theorem 4.1. The topological entropy function

$$
h: C^{\infty}(I, I) \rightarrow[0, \infty)
$$

is continuous.

Proof. Lower semi-continuity of the entropy, for interval maps, has been proved by Misiurewicz [Mis2]. (See also [ALM].) In fact he even proved lower semi-continuity for $C^{0}$-maps with the $C^{0}$-topology. Upper semi-continuity, for $C^{\infty}$-maps in any dimension, 
has been proved by Yomdin [Y]. Finally, the statement that $h(f)<\infty$ is true for any $C^{1}$ map of a compact manifold, and follows from an easily verified bound which takes the form

$$
h(f) \leq \log ^{+} \max _{x}\left|f^{\prime}(x)\right|
$$

in the 1-dimensional case. (Here $\log ^{+}(s)$ is defined to be the maximum of $\log (s)$ and zero.)

The following immediate consequence of this theorem is essential for our purpose:

Corollary 4.2. For any $d$, the topological entropy function is continuous on the finite dimensional compact space consisting of all polynomial maps of the interval with degree $\leq d$.

Remarks. It is essential for Yomdin's Theorem that we work with the $C^{\infty}$ topology. In fact Misiurewicz and Szlenk have shown that $h: C^{r}(I, I) \rightarrow[0, \infty)$ is not upper semicontinuous for any $r<\infty$. However, their example involves a sequence of $m$-modal maps with $m \rightarrow \infty$, converging to a bimodal limit. Misiurewicz [Mis4] has recently proved the following statement, which is much sharper than 4.2: If $\mathcal{M}_{\ell}^{1}$ is the space of $C^{1}$-smooth maps of the interval $I$ which are $m$-modal for some $m \leq \ell-1$, with the $C^{1}$-topology, then $h: \mathcal{M}_{\ell}^{1} \rightarrow[0, \log (\ell)]$ is continuous. Note that Misiurewicz' s lower semi-continuity result for entropy is true only in dimension one. As an example ([Mis1]), consider the family of maps

$$
M_{t}(x, y)=(4 x y(1-x), t y)
$$

of the unit square. Here $h\left(M_{1}\right)=\log (2)$, but $h\left(M_{t}\right)=0$ for $t<1$. In the case of diffeomorphisms of class $C^{1+\epsilon}$, Katok [Ka] has proved an analogous lower semi-continuity theorem in dimension 2, but again there are easy counterexamples in higher dimensions. For further information about continuity properties of the topological entropy function, see also [Mis3] and [N], as well as [D].

Consider a family of maps $f_{\mathbf{p}}$ parameterized by some compact space $P$, and suppose that the topological entropy function $\mathbf{p} \mapsto h\left(f_{\mathbf{p}}\right)$ is continuous, with values in some interval $[\alpha, \beta]$.

Definition. For each fixed $h_{0} \in[\alpha, \beta]$, the $h_{0}$-isentrope for this family is defined to be the set consisting of all parameter values $\mathbf{p} \in P$ for which the topological entropy $h\left(f_{\mathbf{p}}\right)$ is equal to $h_{0}$.

Evidently the various isentropes are disjoint compact subsets, with union equal to $P$. Our goal is to show that all isentropes in the cubic family are connected. (Compare Figure 8. In fact countably many of these isentropes are connected regions with interior, while one, with $h_{0}=\log (3)$, is a single point. It seems possible that all of the rest are simple arcs.)

The rest of this section will outline basic results about the topological entropy of multimodal maps, which we will need in order to describe the structure of isentropes. First a 
fundamental formula proved by Misiurewicz and Szlenk and also by Rothschild: For any piecewise monotone map, we have

$$
h(f)=\lim _{k \rightarrow \infty} \frac{\log \ell\left(f^{\circ k}\right)}{k}=\inf _{k>0} \frac{\log \ell\left(f^{\circ k}\right)}{k},
$$

where $\ell$ is the lap number, as defined in $\S 1$ or $\S 2$. (Compare [MSz], [Ro], [ALM].) It follows that

$$
0 \leq h(f) \leq \log \ell(f) .
$$

In particular, $h(f) \leq \log (m+1)$ if $f$ is $m$-modal.

In practice, it is often more convenient to work with the quantity

$$
\gamma=\exp (h)=\lim _{n \rightarrow \infty} \sqrt[n]{\ell\left(f^{\circ n}\right)},
$$

known as the growth number of $f$. For a polynomial map of degree $d>0$, note that this number $\gamma$ lies in the closed interval $[1, d]$. In the special case of a piecewise linear map with $\mid$ slope $\mid=$ constant $\geq 1$, the growth number $\gamma$ is precisely equal to this constant |slope|. (Compare $[\mathrm{MSz}] .^{5}$ )
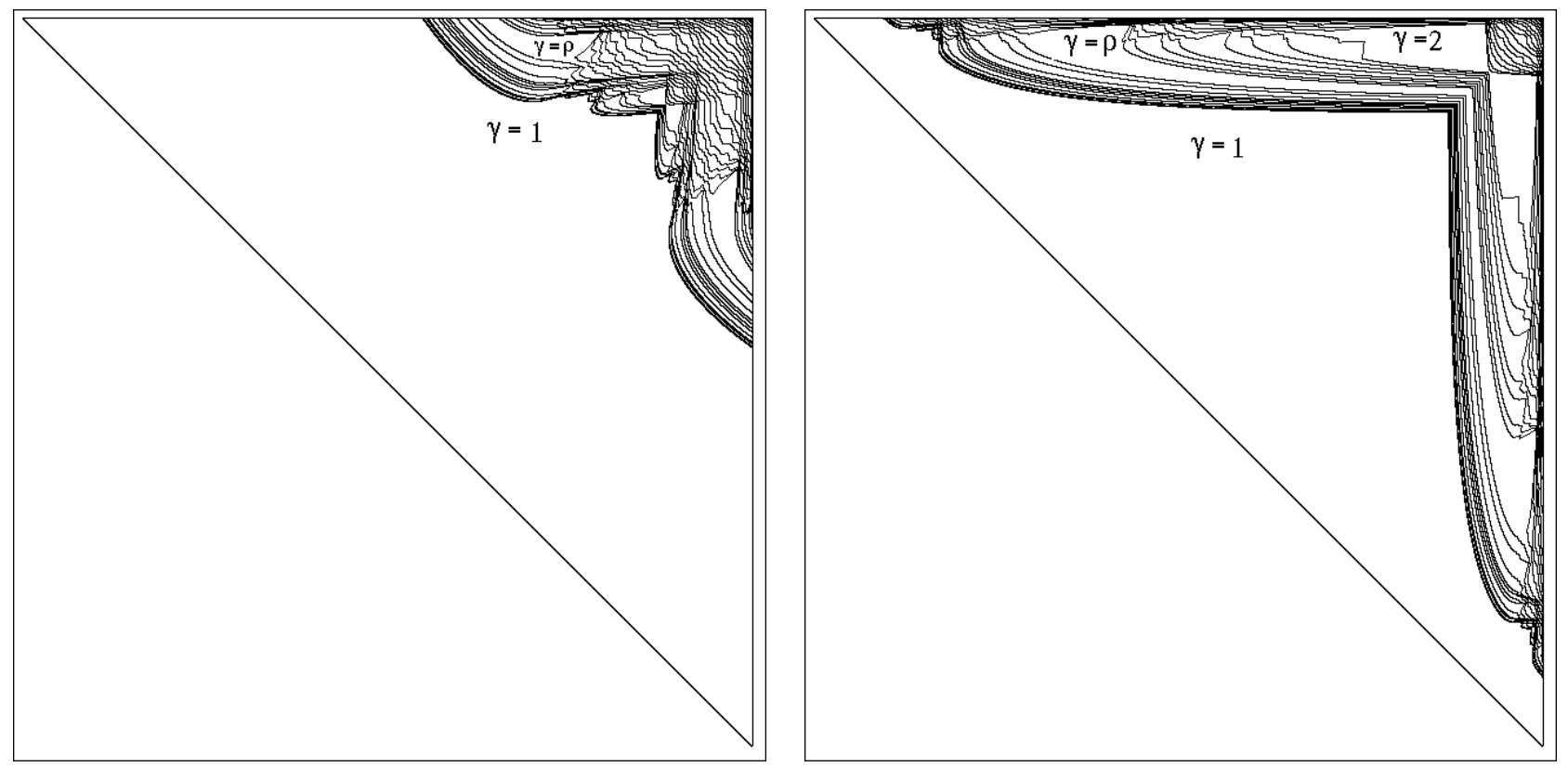

Figure 8. Isentropes $h=\log (\gamma)=$ constant in the parameter triangle $P^{2}$ for real cubic maps of shape $(+-+)$ on the left and $(-+-)$ on the right. Here $\rho$ stands for the golden ratio $(1+\sqrt{5}) / 2$, associated with an attracting period 3 orbit with both critical points in its immediate basin. Similarly, the value $\gamma=2$ is associated with a "capture" component, with say $c_{1}$ and $f^{\circ 2}\left(c_{2}\right)$ in the immediate basin of an attracting fixed point. Compare [M1, App. B].

\footnotetext{
${ }^{5}$ Here is is essential that $f$ be piecewise monotone. The map $F(x)=\inf \left\{3\left|x-1 / 2^{n}\right| ; n \geq 0\right\}$ on the unit interval has $\mid$ slope $\mid=3$ almost everywhere, and yet has $h(F)=0$ since $F(x) \leq x$ for all $x$.
} 
The proof of (8) actually yields a slightly sharper statement as follows. Let us say that a cover $\mathfrak{C}$ of the interval $I$ is $f$-mono if $\mathfrak{C}$ consists of finitely many (possibly degenerate) subintervals, and if $f$ is monotone on each of these subintervals. Evidently such a cover exists if and only if $f$ is piecewise monotone. According to [MSz] (or [ALM], Prop.4.2.3), we have

$$
h(f)=h(f, \mathfrak{C}) \quad \text { whenever the cover } \mathfrak{C} \text { is } f \text {-mono . }
$$

In particular, interpreting the alphabet $\mathfrak{A}$ of $\S 2$ as an $f$-mono cover of the interval $I$, we have $h(f)=h(f, \mathfrak{A})$. (Compare 4.4 below.)

One well known consequence of (9) is an easy computation of $\gamma$, or of $h=\log (\gamma)$, in the case of a map where the orbits of all of the folding points are finite. For such maps, the forward orbits of the folding points cut the interval $I$ into finitely many subintervals. Numbering these subintervals in their natural order as $J_{1}, \ldots, J_{n}$, the associated $n \times n$ Markov transition matrix $M$ is defined by setting

$$
M_{i j}= \begin{cases}+1 & \text { if } f\left(J_{i}\right) \supset J_{j}, \\ 0 & \text { otherwise. }\end{cases}
$$

Recall that a real or complex number $\gamma$ is called an algebraic integer if it is a root of a monic polynomial equation with integer coefficients, and an algebraic unit if both $\gamma$ and $1 / \gamma$ are algebraic integers.

Lemma 4.3. If $f$ is a piecewise monotone map such that the orbits of the folding points are eventually periodic, then the topological entropy of $f$ has the form $h=\log (\gamma)$ where $\gamma$ is the largest real eigenvalue of the associated Markov transition matrix. In particular, $\gamma$ is an algebraic integer. Furthermore

$$
h \leq \limsup _{k \rightarrow \infty} \frac{\log \left(\# \operatorname{fix}\left(f^{\circ k}\right)\right)}{k},
$$

where \# $\operatorname{fix}\left(f^{\circ k}\right)$ denotes the number of fixed points of $f^{\circ k}$. If all of the folding points are actually periodic, then $\gamma$ is an algebraic unit.

(Compare 4.8 and 4.10.) See [BST] as well as 5.10 for methods of computing $h$ in more general cases, based on this formula $h=\log \gamma$.

Outline Proof of 4.3. It is convenient to use the sum of absolute values norm $\|A\|=$ $\sum\left|A_{i j}\right|$ for any $n \times n$ real or complex matrix. If $\lambda_{1}, \ldots \lambda_{n}$ are the eigenvalues of $A$, and if $\gamma$ is the maximum of $\left|\lambda_{i}\right|$, then careful matrix estimates show that

$$
\log \gamma=\lim _{k \rightarrow \infty}\left(\log \left\|A^{k}\right\|\right) / k=\limsup _{k \rightarrow \infty}\left(\log \left|\operatorname{trace}\left(A^{k}\right)\right|\right) / k .
$$

We will apply these equations to the Markov matrix $M$. In this case, it follows from the Perron-Frobenius Theorem, that the number $\gamma=\max \left|\lambda_{j}\right|$ is itself an eigenvalue, and hence can be described as the largest real eigenvalue of $M$. Let us say that a finite or infinite sequence $\left(J_{i_{0}}, J_{i_{1}}, \ldots\right)$ of subintervals $J_{i_{k}}$ is $M$-admissible if $f\left(J_{i_{k}}\right) \supset J_{i_{k+1}}$ for all appropriate $k$. It is easy to check that the number of sequences $\left(J_{i_{0}}, J_{i_{1}}, \ldots, J_{i_{\ell}}\right)$ of length $\ell+1$ which are $M$-admissible is precisely equal to the norm $\left\|M^{\ell}\right\|$. These facts, together with equation (9) for the covering $\left\{J_{i}\right\}$, imply that $h=\log \gamma$. Similarly, 
the number of infinite $M$-admissible sequences which are periodic of period dividing $k$ is equal to the trace of $M^{k}$. Since every $M$-admissible sequence of $J_{i}$ with period dividing $k$ corresponds to at least one periodic point $x=f^{\circ k}(x)$, and since this sequence is usually uniquely determined by $x$, the required inequality for periodic points follows.

Now suppose that every folding point of $f$ belongs to a periodic orbit. List the points $x_{1}<x_{2}<\cdots<x_{n+1}$ which belong to the orbits of folding points. The action of $f$ on these points is described by an $(n+1) \times(n+1)$ permutation matrix $A$, where $A_{i j}=1$ if and only if $f\left(x_{i}\right)=x_{j}$. The associated Markov transition matrix $M$ between the intervals $\left[x_{i}, x_{i+1}\right]$ can be contructed out of $A$ in four steps, as follows. Let $A^{\prime}$ be the matrix obtained from $A$ by adding ones to the left of every one. (For a rough bar-graph of the map, rotate this matrix $90^{\circ}$ counterclockwise.) Let $A^{\prime \prime}$ be obtained from $A^{\prime}$ by replacing every row $R_{i}$ except the first by $R_{i}-R_{i-1}$, and let $A^{\prime \prime \prime}$ be the smaller matrix which is obtained from $A^{\prime \prime}$ by deleting its first row and first column. Then it is not difficult to check that all four of these matrices have the same determinant \pm 1 . Note that the $i$-th row of $A^{\prime \prime \prime}$ is negative if and only if $f$ is decreasing on the interval $\left[x_{i}, x_{i+1}\right]$. The required Markov matrix $M$, with $M_{i j}=1$ if and only if the image of the interval $\left[x_{i}, x_{i+1}\right]$ contains $\left[x_{j}, x_{j+1}\right]$, can now be obtained from $A^{\prime \prime \prime}$ by changing the sign of every negative row, so that $M_{i j}=\left|A_{i j}^{\prime \prime \prime}\right|$. Since $M$ is a matrix of integers with determinant \pm 1 , it follows that every eigenvalue is an algebraic unit. In particular, the largest eigenvalue $\gamma$ must be an algebraic unit. This computes the topological entropy of $f$ restricted to the interval $\left[x_{1}, x_{n+1}\right]$. The actual interval of definition for $f$ may be strictly larger than $\left[x_{1}, x_{n+1}\right]$. However, it is not difficult to check that the portion of this interval to the left of $x_{1}$ or to the right of $x_{n+1}$ makes no contribution to $h$. (Compare the proof of 4.4 below.)

Remark. Let $\left(L_{1}, \ldots, L_{n}\right)$ be an eigenvector for $M$, so that $\sum_{j} M_{i j} L_{j}=\gamma L_{i}$. If the $L_{i}$ are strictly positive, then we can construct a piecewise linear "model" for $f$, having slope $\pm \gamma$ everywhere, as follows. Replace each $J_{i}$ by an interval of length $L_{i}$, so that $f\left(J_{i}\right)$ will be replaced by an interval of length $\gamma L_{i}$. Interpolating linearly we obtain the required piecewise linear map, which has the same kneading data as $f$.

As another application of (9), we will prove the following.

Lemma 4.4. The topological entropy of an $m$-modal map $(f, \mathbf{c})$ is determined by its kneading data.

Proof. Assume first that $f: I \rightarrow I$ is boundary anchored. Recall that an itinerary $\mathcal{I}(x)=\left(A_{0}, A_{1}, \ldots\right)$ is called acritical if each $A_{i}$ belongs to the subalphabet $\mathfrak{A}_{0}=$ $\left\{I_{0}, \ldots, I_{n}\right\}$.

Definition: For each $k>0$, let $\operatorname{Adm}(f, k)$, or more precisely $\operatorname{Adm}(f, \mathbf{c}, k)$, be the number of acritical sequences of length $k$ which are admissible for $f$. According to Corollary 2.4, these numbers $\operatorname{Adm}(f, k)$ are determined by the kneading data for $f$. On the other hand, it is not difficult to check that

$$
\ell\left(f^{\circ k}\right) \leq \operatorname{Adm}(f, k) \leq \operatorname{Card}\left(\mathfrak{A}_{f}^{k}\right) .
$$

(In fact $\ell\left(f^{\circ k}\right)=\operatorname{Adm}(f, k)$ in the piecewise strictly monotone case.) Taking the logarithm 
of these quantities, dividing by $k$, and then letting $k \rightarrow \infty$, we see that

$$
h(f) \leq \lim _{k \rightarrow \infty} \frac{1}{k} \log (\operatorname{Adm}(f, k)) \leq h(f)
$$

by (8) and (9), hence

$$
h(f)=\lim _{k \rightarrow \infty} \frac{1}{k} \log (\operatorname{Adm}(f, k)) .
$$

Thus $h(f)$ is determined by kneading data when $f$ is boundary anchored.

Now consider the more general case where $f: I \rightarrow I$ is not boundary anchored. It is not difficult to extend $f$ to a boundary anchored map $g: J \rightarrow J$ on a strictly larger interval $J \supset I$, where $g$ has the same shape and the same kneading data. Let $L$ and $R$ be the two connected components of $J \backslash I$. (One of these two may be empty.) Since $g(I)=f(I) \subset I$, we see that any orbit for $g$ either lies completely in $I$, or lies completely in $L \cup R$, or else consists of a finite initial segment in $L \cup R$ followed by a terminal segment in $I$. Note also that neither $g(L)$ nor $g(R)$ can intersect both $L$ and $R$. Hence there are only two possible sequence of any given length in $L \cup R$. It follows that

$$
\operatorname{Card}\left(\mathfrak{A}_{g}^{k}\right) \leq 2 \sum_{i=0}^{k} \operatorname{Card}\left(\mathfrak{A}_{f}^{i}\right) .
$$

Now for any constant $\log (c)>h(f)$ we have $\left(\log \operatorname{Card}\left(\mathfrak{A}_{f}^{k}\right)\right) / k<\log (c)$ or equivalently $\operatorname{Card}\left(\mathfrak{A}_{f}^{k}\right)<c^{k}$, for large $k$. Hence there is a constant $a$ so that $\operatorname{Card}\left(\mathfrak{A}_{f}^{k}\right)<a c^{k}$ for all $k$. It then follows that

$$
\operatorname{Card}\left(\mathfrak{A}_{g}^{k}\right)<2 a\left(1+c+\cdots+c^{k}\right)<a^{\prime} c^{k}
$$

for some constant $a^{\prime}$. Therefore $h(g)<\log (c)$ for all such $c$. This implies that $h(g) \leq h(f)$, hence $h(g)=h(f)$, as required.

Using these results, we can define a useful partial order on the possible kneading data $\mathbf{K}(f)$ for $m$-modal maps of a given shape. Let us say that $\mathbf{K}(f) \gg \mathbf{K}(g)$ if and only if

$$
\begin{aligned}
& \mathcal{K}_{i}(f) \geq \mathcal{K}_{i}(g) \text { when } \quad \sigma_{i}=-1, \\
& \mathcal{K}_{i}(f) \leq \mathcal{K}_{i}(g) \text { when } \sigma_{i}=+1,
\end{aligned}
$$

for $1 \leq i \leq m$.

$$
\text { Corollary 4.5. If } \mathbf{K}(f) \gg \mathbf{K}(g) \text {, then } h(f) \geq h(g) \text {. }
$$

Proof. If $\mathbf{K}(f) \gg \mathbf{K}(g)$, then it is easy to check that every admissible sequence for $g$ is also admissible for $f$. Therefore $\operatorname{Adm}(f, k) \geq \operatorname{Adm}(g, k)$ (with notation as in the proof of 4.4). Making use of formula (10), the conclusion follows.

This corollary will help us to reduce questions about isentropes to questions about kneading data. More precisely, our strategy will develop as follows: we will first verify the Connected Isentrope Conjecture for the special family of "stunted sawtooth" maps, and then transfer as much as we can of parameter space information from that family to the cubic family. 
We will see in 5.8 that topological entropy depends continuously on the kneading data. Here is a preliminary result in that direction. We give the space $\mathfrak{A}^{\mathbb{N}}$ of itineraries the usual infinte product topology.

Lemma 4.6. Topological entropy is upper semi-continuous as a function of kneading data.

Proof. Let $f: I \rightarrow I$ be a map with given kneading data, and let $g_{k}: I \rightarrow I$ be any sequence of maps with the same shape such that the truncations of corresponding kneading sequences of $f$ and $g_{k}$ agree up to length $k$. It follows that $\operatorname{Adm}\left(f, k^{\prime}\right)=\operatorname{Adm}\left(g_{k}, k^{\prime}\right)$ for $k^{\prime} \leq k$. Given $\epsilon>0$, it follows from (10) that we can choose $k_{0}$ large enough so that $\left(\log \operatorname{Adm}\left(f, k_{0}\right)\right) / k_{0}<h(f)+\epsilon$. Then for $k \geq k_{0}$ we have

$$
h\left(g_{k}\right) \leq\left(\log \operatorname{Adm}\left(g_{k}, k_{0}\right)\right) / k_{0}=\left(\log \operatorname{Adm}\left(f, k_{0}\right)\right) / k_{0}<h(f)+\epsilon,
$$

as required.

Corollary 4.7. Let $f$ be a piecewise monotone map whose kneading sequences are all acritical, so that the orbit of a folding value never meets a folding point. Then the topological entropy is continuous at $f$ under $C^{0}$ deformations which move the folding points continuously, keeping these points separated and keeping their number fixed.

Proof. Upper semi-continuity follows easily from Lemma 4.6. Lower semi-continuity holds true at all continuous interval maps by [Mis2]. (In fact here, the same result for piecewise monotone maps, as proved in $[\mathrm{MSz}]$, would suffice.)

For one-dimensional maps, there is a close relationship between topological entropy and the existence of periodic orbits. Let us define

$$
h_{\text {per }}(f)=\limsup _{k \rightarrow \infty} \frac{\log \# \operatorname{fix}\left(f^{\circ k}\right)}{k},
$$

where \#fix is the number of fixed points. (Compare 4.3.)

Caution. It is essential to take the lim sup since the limit may well not exist. As an example, consider the tent map $T_{s}(x)=s \max (x, 1-x)$, with slope $s=\sqrt{2}$ chosen so that the two subintervals $[\sqrt{2}-1,2-\sqrt{2}]$ and $[2-\sqrt{2}, \sqrt{2} / 2]$ map to each other. Then it is not difficult to check that

$$
\# \operatorname{fix}\left(T_{s}^{\circ k}\right)= \begin{cases}2 & \text { for } k \text { odd } \\ 2^{k / 2+1} & \text { for } k \text { even. }\end{cases}
$$

Hence $\left(\log \# \operatorname{fix}\left(T_{s}^{\circ k}\right)\right) / k$ tends to zero as $k$ tends to infinity through odd integers, but tends to the limit $h_{\text {per }}\left(T_{s}\right)=\log \sqrt{2}$ as $k$ tends to infinity through even integers.

Lemma 4.8 (Misiurewicz and Szlenk). The inequality $h_{\mathrm{per}}(f) \geq h(f)$ is valid for any piecewise monotone map.

Compare [MSz]. In fact Misiurewicz later showed that this inequality is valid for any continuous interval map (compare [Mis2] or [ALM, 4.3.14]). However, the piecewise monotone case will suffice for our purposes. (Compare 4.12.) 
In fact, equality holds in many important cases. See 4.10 below.

We will need to subdivide periodic orbits into three classes. Recall that every periodic point, $f^{\circ p}(x)=x$ has an itinerary $\mathcal{I}(x)=\left(A_{0}, A_{1}, \ldots\right)$ which is also periodic, with $A_{i}=$ $A_{i+p}$. Define the sign of this fixed point of $f^{\circ p}$ to be the product $\epsilon\left(A_{0}\right) \epsilon\left(A_{1}\right) \cdots \epsilon\left(A_{p-1}\right)$, where

$$
\epsilon\left(I_{j}\right)=\sigma_{j} \in\{ \pm 1\} \quad \text { and } \quad \epsilon\left(C_{j}\right)=0
$$

as in $\S 2$. (In the special case where $f$ is piecewise strictly monotone, this sign is either $+1,-1$, or zero according as $f^{\circ p}$ is increasing, decreasing, or has a folding point at $x$.) A periodic orbit will be called of positive, negative, or critical type according as its sign is $+1,-1$, or 0 . Similarly, a periodic sequence in $\mathfrak{A}^{\mathbb{N}}$ has either positive, negative or folding type. We will be particularly interested in periodic points of negative type, since they are quite stable under perturbation of the $m$-modal map $(f, \mathbf{c})$. In particular, we have the following, with notation as in 4.5.

Lemma 4.9. Given any admissible symbol sequence $\left\{A_{i}\right\}$ which is periodic of negative type, with $A_{i}=A_{i+p}$, there is one and only one fixed point of $f^{\circ p}$ which has this symbol sequence as itinerary. Hence the number $\operatorname{Neg}\left(f^{\circ p}\right)$ of fixed points of negative type for each iterate $f^{\circ p}$ is completely determined by the kneading data for $(f, \mathbf{c})$, and satisfies $\operatorname{Neg}\left(f^{\circ p}\right) \leq \operatorname{Adm}(f, p)$. Furthermore, if $\mathbf{K}(f) \gg \mathbf{K}(g)$, then $\operatorname{Neg}\left(f^{\circ p}\right) \geq \operatorname{Neg}\left(g^{\circ p}\right)$ for every $p \geq 1$.

(Note that a fixed point of negative type for $f^{\circ p}$ counts also as a fixed point of negative type for the odd iterates $f^{\circ 3 p}, f^{\circ 5 p}, \ldots$, but as a fixed point of positive type for $f^{\circ 2 p}, f^{\circ 4 p}, \ldots$ )

Proof of 4.9. Let $J=(\alpha, \beta) \subset I$ be the subinterval consisting of all $x \in I$ with $A\left(f^{\circ i}(x)\right)=A_{i}$ for $0 \leq i<p$. Then the restriction $\left.f^{\circ p}\right|_{J}$ is monotone decreasing with $f^{\circ p}(J) \cap J \neq \emptyset$. This implies that $f(\alpha)>\alpha$ and $f(\beta)<\beta$. Hence by the Intermediate Value Theorem, $f^{\circ p}{ }_{J}$ has a fixed point, which is unique since this restriction is monotone decreasing. Thus $\operatorname{Neg}\left(f^{\circ p}\right)$ is equal to the number of admissible sequences which are fixed points of negative type for the $p$-fold iterate of the shift. It follows that $\operatorname{Neg}\left(f^{\circ p}\right) \leq$ $\operatorname{Adm}(f, p)$. In the boundary anchored case, the statement that the numbers $\operatorname{Neg}\left(f^{\circ p}\right)$ are completely determined by the kneading data follows immediately from Corollary 2.4. The general case follows since, extending $f$ to a boundary anchored map as in the proof of 4.4 , it is easy to see that no periodic orbit of negative type can involve either of the two intervals which are adjoined to the ends of $I$.

Lemma 4.10. If $f$ is piecewise monotone, with at most finitely many nonrepelling periodic orbits, then

$$
h(f) \geq h_{\mathrm{per}}(f)=\limsup _{k \rightarrow \infty} \frac{\log ^{+} \operatorname{Neg}\left(f^{\circ k}\right)}{k} .
$$

Combining this inequality with 4.8 , it follows of course that

$$
h(f)=h_{\text {per }}(f)=\limsup _{k \rightarrow \infty} \frac{\log ^{+} \operatorname{Neg}\left(f^{\circ k}\right)}{k}
$$


whenever $f$ has at most finitely many non-repelling periodic orbits. This hypothesis is satisfied in many important cases. For example, for a polynomial map of degree $d>1$, the classical theory of Fatou and Julia shows that the number of such orbits is at most $d-1$. For a smooth ${ }^{6} m$-modal map with negative Schwarzian derivative, the number of such orbits is at most $m+2$. For an $m$-modal stunted sawtooth map with $m>1$, as studied in $\S 5$, the number of such orbits is at most $m$.

Remark. This close relationship between topological entropy and periodic orbits exists only in low dimensions. Katok [Ka] has proved the inequality $h_{\text {per }} \geq h$ for 2-dimensional diffeomorphisms which are $C^{1+\alpha}$-smooth. However, Kaloshin $[\mathrm{K}]$ has shown that $h_{\text {per }}$ is infinite (and hence strictly greater that $h$ ) for $C^{r}$-generic maps inside a "Newhouse region" in parameter space. As soon as we go to higher dimensions or allow non-smooth maps, there can be maps of positive entropy with no periodic orbits at all. As an example, the cartesian product of an irrational rotation of the circle with an arbitrary dynamical system for which $h>0$ will have $h>0$, but no periodic orbits. For the case of non-smooth surface homeomorphisms, see Rees [Re].

Outline Proof of 4.10. First suppose that all of the periodic orbits of $f$ are nonfolding and strictly repelling. Then evidently the fixed points of $f^{\circ p}$, that is the places where the graph of $f^{\circ p}$ crosses the diagonal, must be alternately of positive and negative type, and it follows easily that

$$
\left|\# \operatorname{fix}\left(f^{\circ p}\right)-2 \mathrm{Neg}\left(f^{\circ p}\right)\right| \leq 1 .
$$

If there are $\ell$ periodic points which are either of folding type or are non-repelling, then a similar argument shows that

$$
\left|\# \operatorname{fix}\left(f^{\circ p}\right)-2 \operatorname{Neg}\left(f^{\circ p}\right)\right| \leq 2 \ell+1 .
$$

Whenever this number $\ell$ is finite, it follows immediately that

$$
h_{\text {per }}(f)=\limsup _{k \rightarrow \infty}\left(\log ^{+} \operatorname{Neg}\left(f^{\circ k}\right)\right) / k,
$$

and since $\operatorname{Neg}\left(f^{\circ p}\right) \leq \operatorname{Adm}(f, p)$ by 4.9 , it follows by $(10)$ that $h_{\text {per }} \leq h$.

Main Theorem 4.11. For any m-modal map, we have

$$
h(f)=\limsup _{k \rightarrow \infty} \frac{1}{k} \log ^{+}\left(\operatorname{Neg}\left(f^{\circ k}\right)\right) .
$$

This is proved in [MTh], and also in $[\mathrm{Pr}]$. (Either one of these references describes explicitly how to compute these numbers $\operatorname{Neg}\left(f^{\circ k}\right)$ from the kneading data.)

Remark 4.12. These results are closely related. Thus 4.8 is an immediate corollary of 4.11 , since \#fix $\left(f^{\circ p}\right) \geq \operatorname{Neg}\left(f^{\circ p}\right)$. On the other hand, a proof that 4.11 follows from 4.8 can be sketched as follows. If $f$ has only finitely many non-repelling periodic orbits, then 4.11 follows immediately from 4.8 and 4.10 . However, we know by 4.4 that the topological entropy $h(f)$ is completely determined by the kneading data for $f$, and we know by 4.9 that the numbers $\operatorname{Neg}\left(f^{\circ p}\right)$ are completely determined by the kneading data for $f$. Hence,

\footnotetext{
${ }^{6}$ Note also the following theorem of Martens, de Melo and van Strien [MMS]: For any $C^{1}$-smooth interval map with non-flat critical points, every orbit of sufficiently high period is repelling.
} 
if we can find just one example of a map $g$ which has the same kneading data as $f$, and which has only finitely many non-repelling periodic orbits, then 4.11 will follow. In fact such an example will be provided in the next section (5.3 together with 5.4).

Another proof of both 4.8 and 4.11 will be described at the end of $\S 5$.

Define the negative orbit complexity of $f$ to be the sequence

$$
\mathcal{N}(f)=\left(\operatorname{Neg}\left(f^{\circ 1}\right), \operatorname{Neg}\left(f^{\circ 2}\right), \operatorname{Neg}\left(f^{\circ 3}\right), \ldots\right)
$$

of non-negative integers, and define the relation $\mathcal{N}(f) \gg \mathcal{N}(g)$ to mean that $\operatorname{Neg}\left(f^{\circ p}\right) \geq$ $\operatorname{Neg}\left(g^{\circ p}\right)$ for all $p$, then we can summarize $4.5,4.9 \mathrm{~b}$, and 4.11 as follows.

Corollary 4.13. The kneading data $\mathbf{K}(f)$ determines the negative orbit complexity $\mathcal{N}(f)$, which in turn determines the topological entropy $h(f)$, with

$$
\mathbf{K}(f) \gg \mathbf{K}(g) \quad \Rightarrow \quad \mathcal{N}(f) \gg \mathcal{N}(g) \quad \Rightarrow \quad h(f) \geq h(g)
$$

\section{$\S 5$. The stunted sawtooth family.}

Closely related to kneading theory is a special family of piecewise monotone maps which is rich enough to encompass in a canonical way all possible kneading data and all possible itineraries. (Compare $[\mathrm{Gu}],[\mathrm{BCMM}]$, as well as Figure 9.) In order to introduce this family, we first introduce the sawtooth map of specified shape $\boldsymbol{\sigma}=\left(\sigma_{0}, \ldots, \sigma_{m}\right)$. This map, on an interval $J$, can be characterized as the unique piecewise linear map $S: J \rightarrow \mathbb{R}$ which is boundary anchored of shape $\boldsymbol{\sigma}$, with slope $\pm s$ everywhere where $s>m+1$ is some specified constant, ${ }^{7}$ and with folding points in arithmetic progression.

Caution: This map $S$ does not carry the interval $J$ into itself. In fact the folding values of $S$ all lie outside of $J$.

The precise choice of the constant slope $s$ doesn't matter, but to fix our ideas, let us always take $s=m+3 / 2$. One choice of the interval $J$ is particularly convenient for kneading theory: As folding points $\widehat{C}_{1}, \ldots, \widehat{C}_{m}$ choose the numbers

$$
-m+1,-m+3, \ldots, m-1,
$$

with $\widehat{C}_{j}=2 j-m-1$, and with $S\left(\hat{C}_{j}\right)=-\sigma_{j} s$ as the corresponding folding values. Now choose a base point $\widehat{I}_{j}=2 j-m$ in the $j$-th lap, so that

$$
\widehat{I}_{0}<\widehat{C}_{1}<\widehat{I}_{1}<\cdots<\widehat{C}_{m}<\widehat{I}_{m}
$$

are consecutive integers. Then $S$ is given by the formula

$$
S(x)=\sigma_{j} s\left(x-\hat{I}_{j}\right) \text { for } \quad x \in I_{j} .
$$

We must choose a domain of definition $J$ for $S$ so that $S(\partial J) \subset \partial J$. The appropriate

\footnotetext{
${ }^{7}$ In the preliminary publication [DGMT] (and also in 2.2) we took $s=m+1$. However, here we will need $s>m+1$ in order to get an actual embedding of the set of all $\mathbf{K}$-admissible sequences in $\mathfrak{A}^{\mathbb{N}}$ into the reals.
} 

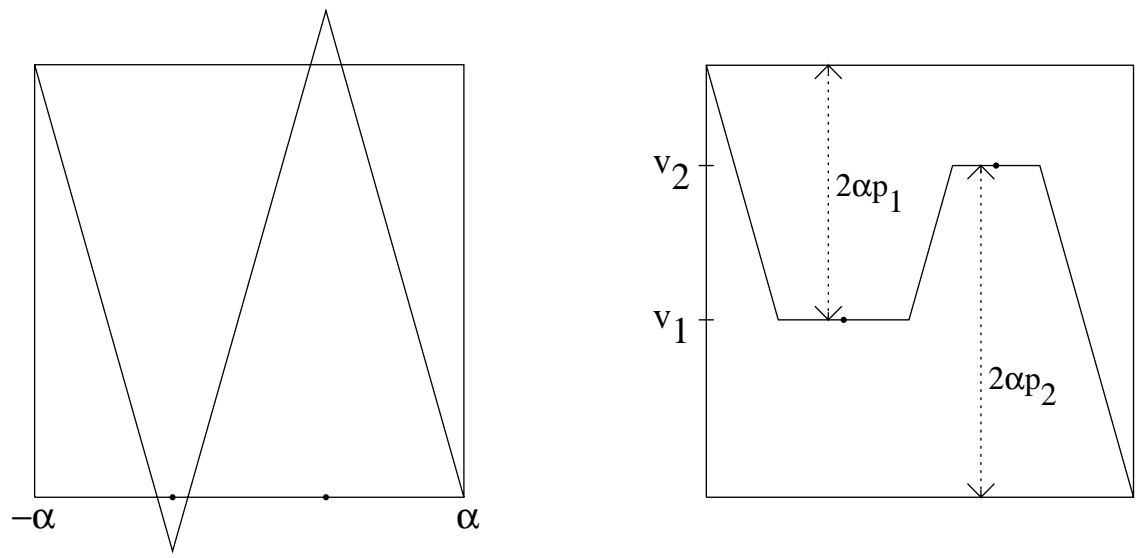

Figure 9. The bimodal sawtooth map of shape $(-+-)$, and a representative stunted sawtooth map of the same shape.

choice is the interval $J=[-\alpha, \alpha]$, where

$$
\alpha=\frac{m s}{s-1}<m+1<s .
$$

Since $\alpha=s(\alpha-m)$, it follows that $S( \pm \alpha) \in\{ \pm \alpha\}$, as required.

As in $\S 2$, we work with the ordered alphabet $\mathfrak{A}=\left\{I_{0}, C_{1}, I_{1}, \ldots, C_{m}, I_{m}\right\}$. The correspondence $I_{j} \mapsto \widehat{I}_{j}, \quad C_{j} \mapsto \widehat{C}_{j}$, or briefly $A \mapsto \widehat{A}$ for $A \in \mathfrak{A}$, defines an order preserving embedding of this alphabet into the integers. We can extend to a map $\Phi: \mathfrak{A}^{\mathbb{N}} \rightarrow \mathbb{R}$ by setting

$$
\begin{aligned}
\Phi\left(A_{0}, A_{1}, \ldots\right) & =\widehat{A}_{0}+\epsilon_{0} \widehat{A}_{1} / s+\epsilon_{0} \epsilon_{1} \widehat{A}_{2} / s^{2}+\cdots \\
& =\sum_{k=0}^{\infty} \epsilon_{0} \cdots \epsilon_{k-1} \widehat{A}_{k} / s^{k} .
\end{aligned}
$$

(Recall that $\epsilon_{i}=\epsilon\left(A_{i}\right)$ with $\epsilon\left(I_{j}\right)=\sigma_{j}$ and $\epsilon\left(C_{j}\right)=0$.) Order the space of sequences $\mathfrak{A}^{\mathbb{N}}$ as in $\S 2$.

Lemma 5.1. The image $\Phi\left(\mathfrak{A}^{\mathbb{N}}\right)$ is contained in the interval $J=[-\alpha, \alpha]$, and $\Phi$ is related to the sawtooth map on $J$ by the identity

$$
\Phi\left(A_{1}, A_{2}, A_{3}, \ldots\right)=S\left(\Phi\left(A_{0}, A_{1}, A_{2}, \ldots\right)\right)
$$

whenever $\epsilon_{0} \neq 0$, or in other words whenever $A_{0} \in\left\{I_{0}, I_{1}, \ldots, I_{m}\right\}$. (Thus $\Phi$ semiconjugates the shift map on $\mathfrak{A}^{\mathbb{N}}$ to the sawtooth map on $J$, except when $A_{0}$ is a folding point symbol.) This map $\Phi: \mathfrak{A}^{\mathbb{N}} \rightarrow J$ is strictly order preserving, provided that we consider only sequences which satisfy the first compatibility condition of §2 with some fixed kneading data.

Proof of 5.1. Since $\left|\widehat{A}_{j}\right| \leq m$, we have

$$
\left|\Phi\left(A_{0}, A_{1}, \ldots\right)\right| \leq m\left(1+1 / s+1 / s^{2}+\cdots\right)=\alpha,
$$


hence $\Phi\left(\mathfrak{A}^{\mathbb{N}}\right) \subset J$. We can write equation (14) as

$$
\Phi\left(A_{0}, A_{1}, \ldots\right)=\widehat{A}_{0}+\epsilon\left(A_{0}\right) \Phi\left(A_{1}, A_{2}, \ldots\right) / s .
$$

If $\epsilon\left(A_{0}\right)= \pm 1$, then equation $\left(14^{\prime}\right)$ can be solved for

$$
\Phi\left(A_{1}, A_{2}, \ldots\right)=s \epsilon\left(A_{0}\right)\left(\Phi\left(A_{0}, A_{1}, \ldots\right)-\widehat{A}_{0}\right) .
$$

Comparing (14), we can write the right hand side of this equation as $S\left(\Phi\left(A_{0}, A_{1}, \ldots\right)\right)$, as required. Finally, we must show that the map $\Phi: \mathfrak{A}^{\mathbb{N}} \rightarrow J$ is strictly monotone, in the sense that

$$
\left(A_{0}, A_{1}, \ldots\right)<\left(B_{0}, B_{1}, \ldots\right) \Longleftrightarrow \Phi\left(A_{0}, A_{1}, \ldots\right)<\Phi\left(B_{0}, B_{1}, \ldots\right),
$$

provided that both sequences satisfy the First Compatibility Condition of $\S 2$ with the same kneading data. Let $k \geq 0$ be the smallest index with $A_{k} \neq B_{k}$. It follows from $\left(15^{\prime}\right)$ that

$$
\left.\mid \Phi\left(A_{0}, \ldots\right)-\widehat{A}_{0}\right) \mid \leq \alpha / s<1,
$$

with $\Phi\left(A_{0}, \ldots\right)=\widehat{A}_{0}$ when $A_{0}$ is a folding point symbol. If $k=0$ so that $A_{0}<B_{0}$, then it follows immediately from this inequality that

$$
\Phi\left(A_{0}, A_{1}, \ldots\right)<\Phi\left(B_{0}, B_{1}, \ldots\right) .
$$

Now if $k \geq 1$, then $\epsilon\left(A_{0}\right)$ must be non-zero, and an easy induction on $k$, using $\left(14^{\prime}\right)$, proves the corresponding inequality.

Now suppose that we are given an $m$-modal map $f: I \rightarrow I$ of shape $\boldsymbol{\sigma}$. For any non-folding point $x \in I \subset I$ the itinerary $\mathcal{I}(x) \in \mathfrak{A}^{\mathbb{N}}$ maps to a real number

$$
\theta(x)=\Phi(\mathcal{I}(x)) \in J,
$$

which provides an invariantly defined coordinate for the point $x$ with respect to the map $f$. It follows from 5.1 together with $\S 2$ that $\theta: I \rightarrow J$ is a monotone function, in the sense that

$$
x<y \quad \Rightarrow \quad \mathcal{I}(x) \leq \mathcal{I}(y) \quad \Rightarrow \quad \theta(x) \leq \theta(y) .
$$

(However, $\theta$ is certainly not continuous.) Note that $\theta\left(c_{j}\right)=\widehat{C}_{j}$, and that $\theta(x)=\theta(y)$ if and only if $x$ and $y$ (and hence all points between $x$ and $y$ ) have the same itinerary. For any $x \in I_{j}$ it follows from 5.1 that

$$
\theta(f(x))=s \sigma_{j}\left(\theta(x)-\widehat{I}_{j}\right)=S(\theta(x)) .
$$

Thus $\theta$ is a monotone but discontinuous semiconjugacy from the map $f$ on $I$ to the sawtooth map $S$ on $J$, provided that we exclude folding points. However, this identity $\theta(f(x))=S(\theta(x))$ definitely breaks down when $x=c_{j}$, since $\theta\left(f\left(c_{j}\right)\right) \in J$ but $S\left(\theta\left(c_{j}\right)\right)=$ $S\left(\widehat{C}_{j}\right) \notin J$. To correct this problem, we truncate the map $S$ as follows. Let us specify some folding value vector $\mathbf{v}=\left(v_{1}, \ldots, v_{m}\right) \in J^{m}$, subject to the usual inequalities (2):

$$
\begin{array}{lll}
v_{j} \leq v_{j+1} & \text { when } & \sigma_{j}=+1 \\
v_{j} \geq v_{j+1} & \text { when } & \sigma_{j}=-1,
\end{array}
$$

for $0<j<m$.

Definition. The stunted sawtooth map $S_{\mathbf{p}}: J \rightarrow J$ is obtained by chopping off the 
successive peaks and pits of the map $S$ at the heights $v_{1}, \ldots, v_{m}$, as shown in Figure 9. Thus $S_{\mathbf{p}}$ is a continuous function which takes the constant value $v_{j}$ throughout the largest connected neighborhood of $\widehat{C}_{j}=2 j-m-1$ on which

$$
\begin{array}{lll}
S(y) \geq v_{j} & \text { if } & S\left(\widehat{C}_{j}\right)=+\alpha \\
S(y) \leq v_{j} & \text { if } & S\left(\widehat{C}_{j}\right)=-\alpha,
\end{array}
$$

but $S_{\mathbf{p}}(y)$ is equal to $S(y)$ otherwise.

Note: Here, as in $\S 3$, we parametrize the admissible folding value vectors $\mathbf{v} \in J^{m}$ by vectors $\mathbf{p}$ belonging to a standard polyhedron $P^{m} \subset[0,1]^{m}$. Since $J=[-\alpha, \alpha]$, the transformation $\mathbf{p} \leftrightarrow \mathbf{v}$ of (6) is given by

$$
v_{j}=\left(2 p_{j}-1\right) \sigma_{j} \alpha \in[-\alpha, \alpha] \quad \text { or } \quad p_{j}=\frac{1}{2}+\frac{\sigma_{j} v_{j}}{2 \alpha} \in[0,1] .
$$

It is not difficult to write down a more explicit formula as follows:

$$
S_{\mathbf{p}}(y)= \begin{cases}v_{j} & \text { if }\left|y-\widehat{C}_{j}\right| \leq 1+\sigma_{j} v_{j} / s \quad \text { for some } j, \\ S(y) & \text { otherwise. }\end{cases}
$$

The interval of constancy

$$
\left\{y \in J ;\left|y-\widehat{C}_{j}\right| \leq 1+\sigma_{j} v_{j} / s\right\}
$$

will be called the $j$-th plateau of $S_{\mathbf{p}}$. Since $s>\alpha \geq\left|v_{j}\right|$, these plateaus always have strictly positive length. Note that the heights of these plateaus are just the corresponding folding values.

Caution: If $v_{j}=v_{j+1}$ (or equivalently if $p_{j}+p_{j+1}=1$ ) then the $j$-th and $j+1$-st plateaus have a common endpoint, and together form a longer interval of constancy. More generally, it follows from (16) that the distance between the $j$-th and $j+1$-st plateaus is equal to $\left|v_{j+1}-v_{j}\right| / s \geq 0$.

By definition, the $j$-th designated folding point of $S_{\mathbf{p}}$ is just the point $\widehat{C}_{j}$, which can also be described as the midpoint of the $j$-th plateau. Note that every $S_{\mathbf{p}}$ is boundary anchored, mapping $\partial J=\{ \pm \alpha\}$ into itself by the fixed map

$$
S_{\mathbf{p}}(-\alpha)=-\sigma_{0} \alpha, \quad S_{\mathbf{p}}(+\alpha)=+\sigma_{m} \alpha .
$$

In order to relate this construction to kneading theory, we choose the folding value vector $\mathbf{v}$ by setting $v_{j}=\Phi\left(\mathcal{K}_{j}\right)$, with $\Phi$ as in 5.1. In this way, we prove the following.

Theorem 5.2. To any shape $\boldsymbol{\sigma}$ and any $m$-tuple $\mathbf{K}$ of symbol sequences satisfying the Compatibility Conditions 1, 2, 3 of $\S 2$, there is associated a canonical stunted sawtooth map $S_{\mathbf{p}}$ which has exactly these kneading data. Furthermore a symbol sequence in $\mathfrak{A}^{\mathbb{N}}$ actually occurs as the itinerary of some point under $S_{\mathbf{p}}$ if and only if it is admissible (i.e., satisfies Compatibility Conditions 1 and 2).

Proof. Let $S$ be the $m$-modal sawtooth map with shape $\boldsymbol{\sigma}$, and let $S_{\mathbf{p}}$ be the associated truncated map with folding values $v_{j}=\Phi\left(\mathcal{K}_{j}\right)$. The Third Compatibility Condition guarantees that these folding values satisfy the required inequalities (2). Notice that the 
orbits under $S$ and $S_{\mathbf{p}}$ are identical as long as they do not enter a plateau of $S_{\mathbf{p}}$. Next, using Compatibility Condition 2, it is not difficult to show that the orbit of a folding value can enter the interior of a plateau of $S_{\mathbf{p}}$ only at the folding point in this plateau. It follows that the folding values $v_{j}$ have the same itineraries, up to their first folding point if any occurs on the itinerary, under $S$ and under $S_{\mathbf{p}}$. We then use the First Compatibility Condition to show that the full itinerary of $v_{j}$ under $S_{\mathbf{p}}$ is equal to the given $\mathcal{K}_{j}$. Further details are straightforward, and will be left to the reader.

Remark. The map $S_{\mathbf{1}}$ corresponding to the vector $\mathbf{p}=\mathbf{1}=(1,1, \ldots, 1)$ is the unique stunted sawtooth map of shape $\boldsymbol{\sigma}$ with maximal numbers of periodic orbits and maximal topological entropy. If $X_{\mathbf{1}}$ is the set of points in $J$ whose orbit under $S_{\mathbf{1}}$ meets the various plateaus of $S_{1}$ only at endpoints or midpoint, then we see that each point of $X_{1}$ is uniquely characterized by its itinerary, which can be either a completely arbitrary infinite sequence in $\left\{I_{0}, I_{1}, \ldots, I_{m}\right\}^{\mathbb{N}}$, or any finite sequence of symbols from $\left\{I_{0}, I_{1}, \ldots, I_{m}\right\}$ followed by a folding point symbol $C_{j}$ (with $1 \leq j \leq m$ ), and then followed by either $\mathcal{I}_{\min }$ or $\mathcal{I}_{\max }$ according as $\sigma_{j}$ is +1 or -1 . In particular, given any vector $\mathbf{K}=\left(\mathcal{K}_{1}, \ldots, \mathcal{K}_{m}\right)$ of symbol sequences, there are unique points $v_{j} \in X_{\mathbf{1}}$ such that the itinerary of each $v_{j}$ under $S_{\mathbf{1}}$ coincides with the given $\mathcal{K}_{j}$ up to the first folding point symbol (if any) in this sequence. If the three Compatibility Conditions are satisfied, then taking these points $v_{j} \in X_{\mathbf{1}}$ to be the folding values for a stunted sawtooth map $S_{\mathbf{p}(\mathbf{v})}$, we easily obtain another proof of Theorem 5.2. (Compare [DGMT].)

Corollary 5.3. To any m-modal map $f: I \rightarrow I$ there is associated a canonical stunted sawtooth map $S_{\mathbf{p}}: J \rightarrow J$ which has exactly the same kneading data. Furthermore, the (monotone but discontinuous) correspondence $\theta: I \rightarrow J$ semiconjugates $f$ to $S_{\mathbf{p}}$. That is

$$
\theta(f(x))=S_{\mathbf{p}}(\theta(x))
$$

for all $x$.

The proof is straightforward.

To complete the discussion in Remark 4.12, we also need the following observation.

Lemma 5.4. An $m$-modal stunted sawtooth map can have at most $m$ nonrepelling periodic orbits.

For at most one periodic orbit can intersect any plateau. But any period $q$ orbit which does not meet any of the plateaus must be strictly repelling, with multiplier $\pm s^{q}$.

Remark 5.5. Theorem 5.2 can be reformulated by saying that for any shape $\boldsymbol{\sigma}$, the set of possible kneading data injects canonically into the convex polyhedron $P^{m}$. We will write $\mathbf{K} \mapsto \mathbf{p}(\mathbf{K})$. This allows us to replace the sometimes cumbersome comparisons of symbol sequences by comparisons of numbers. Evidently

$$
\mathbf{K} \gg \mathbf{K}^{\prime} \quad \Longleftrightarrow \quad p_{i}(\mathbf{K}) \geq p_{i}\left(\mathbf{K}^{\prime}\right) \text { for all } i,
$$

with notation as in 4.5 . 
Remark 5.6. Conversely, suppose that $\mathbf{p}, \mathbf{p}^{\prime} \in P^{m}$ satisfy $p_{i} \geq p_{i}^{\prime}$ for all $i$. Then it is not hard to show that $\mathbf{K}\left(S_{\mathbf{p}}\right) \gg \mathbf{K}\left(S_{\mathbf{p}^{\prime}}\right)$, and it follows (or can be shown directly) that $\operatorname{Adm}\left(S_{\mathbf{p}}, k\right) \geq \operatorname{Adm}\left(S_{\mathbf{p}^{\prime}}, k\right)$ for all $k$, that $\mathcal{N}\left(S_{p}\right) \gg \mathcal{N}\left(S_{\mathbf{p}^{\prime}}\right)$, and that $h\left(S_{\mathbf{p}}\right) \geq h\left(S_{\mathbf{p}^{\prime}}\right)$. (Compare 4.4 and 4.13.) Similarly, an easy argument shows that an increase in $p_{i}$ can only increase the number of period $k$ orbits, or the number of fixed points of $S_{\mathbf{p}}^{\circ k}$. (Compare the proof of 6.1.)

Lemma 5.7. The topological entropy of a stunted sawtooth map depends continuously on its parameters, or in other words on its vector of folding values.

Proof. Lower semi-continuity follows immediately from [Mis2], as noted in $\S 4$. To prove upper semi-continuity, we note that the topological entropy $h(f)$ depends only on the mapping $f$, and not on which particular points within the various plateaus are designated as folding points. However, we can choose these folding points to be disjoint from the forward orbits of all the folding values. Upper semi-continuity then follows from 4.6 .

Recall from 4.4 that topological entropy is uniquely determined by kneading data. Combining 5.2 and 5.7 we have the following sharper form of 4.6.

Corollary 5.8. Topological entropy depends continuously on kneading data.

For the canonical model of 5.2 certainly depends continuously on kneading data. (Alternatively, a direct proof of 5.8 could be based on the methods used in [MTh, Lemma 12.3].)

Definition. Recall that a polynomial map is called "hyperbolic" if every critical point lies in the basin of some periodic attractor. To simplify the analogous discussion for a stunted sawtooth map $S_{\mathbf{p}}$, we consider only the case where $S_{\mathbf{p}}$ is strictly $m$-modal; that is, we assume that consecutive folding values are distinct. It is not hard to see that a periodic orbit for such a map $S_{\mathbf{p}}$ is attracting, and remains attracting under perturbation of the map, if and only if it contains an interior point of some plateau, and hence actually absorbs all orbits in a neighborhood. Let us call a strictly $m$-modal $S_{\mathbf{p}}$ hyperbolic if the forward orbit of each folding value eventually lands in the interior of some plateau. Clearly this is an open condition. The generic hyperbolicity property of stunted sawtooth maps can be stated as follows.

Lemma 5.9. The stunted sawtooth maps which are strictly $m$-modal and hyperbolic form a dense open set in the space of all stunted sawtooth maps of specified shape.

Proof of 5.9. Openness is clear. Furthermore, it is clear that the strictly $m$-modal maps are dense. Suppose inductively that every $S_{\mathbf{p}}$ can be approximated by a strictly $m$ modal $S_{\mathbf{q}}$ for which the orbits of the first $k-1$ folding values eventually hit the interior of some plateau. Then we can choose some $\epsilon>0$ so that these conditions will remain true as we change the $k$-th folding value throughout an $\epsilon$-neighborhood. Now choose an integer $n$ so that the product $s^{n} \epsilon$ is greater that the length of the entire interval $J$. As we move 
the folding value $v_{k}$ with unit speed through its $\epsilon$-neighborhood, its forward image $S_{\mathbf{q}}^{\circ n}$ will move with speed $\pm s^{n}$, so long as its orbit does not pass through any plateau. But we have chosen $n$ with $s^{n} \epsilon$ large enough so that this is impossible. Hence some forward image must pass though a plateau; which completes the inductive construction.

Combining 4.3 with the proof of 5.9 , we have the following basic result.

Theorem 5.10. The topological entropy of a piecewise monotone map can be effectively computed to any required degree of accuracy from its kneading data.

Proof. We must produce computable upper and lower bounds for $h(f)$, arbitrarily close to each other. The proof will be by induction on the number $m$ of folding points. To construct a lower bound, first suppose that the kneading data $\mathbf{K}=\mathbf{K}(f)$ satisfies $\mathcal{K}_{i} \neq \mathcal{K}_{i+1}$ for every $i$, so that the associated stunted sawtooth map $S_{\mathbf{p}}$, with $\mathbf{p}=\mathbf{p}(\mathbf{K})$ as in 5.5, has distinct adjacent folding values. Then using the argument above we see easily that there exists a hyperbolic map $S_{\mathbf{p}^{\prime}}$ so that $\mathbf{p}^{\prime}$ is arbitrarily close to $\mathbf{p}$, and with $p_{i}^{\prime} \leq p_{i}$ for all $i$. Here $h\left(S_{\mathbf{p}^{\prime}}\right)$ is effectively computable by 4.3 , and can be chosen arbitrarily close to $h(f)=h\left(S_{\mathbf{p}}\right)$ by 5.7. On the other hand, if $S_{\mathbf{p}}$ has two adjacent plateaus at the same height, then by ignoring the two corresponding critical points it can be considered as an $(m-2)$-modal map, and the conclusion follows by induction. A completely analogous argument produces a computable upper bound

$$
h(f) \leq h\left(S_{\mathbf{p}^{\prime \prime}}\right)
$$

which is arbitrarily close to $h(f)$. One simply chooses $\mathbf{p}^{\prime \prime}$ close to $\mathbf{p}$, with $p_{i}^{\prime \prime} \geq p_{i}$ for all $i$, so that the orbit of each critical value is eventually periodic. In fact if $p_{i}=1$, then the associated orbit is already eventually periodic, while if $p_{i}<1$ then arguing as in 5.9 we can choose $p_{i}^{\prime \prime}$ so that its orbit eventually hits a plateau.

Remarks. A closely related algorithm, more explicitly worked out, is described in [BST]. For the special case of a bimodal map, Block and Keesling [BK] have given a fast algorithm, based on comparison with maps of $\mid$ slope $\mid=$ constant. (This was used for the plots in Figure 8.) Note that the question of effective computability of entropy for more general dynamical systems remains open: compare [HKC].

As a corollary, we can give another proof of two basic results from $\S 4$.

Proof of 4.11 and 4.8. We first show that

$$
h(f)=\limsup _{k \rightarrow \infty} \log ^{+}\left(\mathrm{Neg}\left(f^{\circ k}\right)\right) / k
$$

for every piecewise monotone map. This statement is certainly true for the approximating maps $S_{\mathbf{p}^{\prime}}$ and $S_{\mathbf{p}^{\prime \prime}}$ of 5.10 , by Lemma 4.3 , together with 4.10 and 5.4 . Since

$$
\mathcal{N}\left(S_{\mathbf{p}^{\prime}}\right) \ll \mathcal{N}\left(S_{p}\right)=\mathcal{N}(f) \ll \mathcal{N}\left(S_{\mathbf{p}^{\prime \prime}}\right)
$$

by 4.9 and 5.6 , the corresponding statement for $f$ follows. The inequality $h_{\text {per }}(f) \geq h(f)$ is an immediate corollary. 


\section{$\S 6$. Contractibility of Isentropes for the Stunted Sawtooth Family.}

We consider the family of stunted sawtooth maps of some specified shape $\boldsymbol{\sigma}=\left(\sigma_{0}, \ldots, \sigma_{m}\right)$, which remains fixed throughout this section. Parameterize this family by the standard polyhedron $P^{m}$, consisting of all vectors

$$
\mathbf{p}=\left(p_{1}, \ldots, p_{m}\right) \in[0,1]^{m} \quad \text { satisfying } p_{i}+p_{i+1} \geq 1 \text { for } 1 \leq i<m,
$$

as described in $\S 3$. Let $h_{\sigma}^{\text {saw }}: P^{m} \rightarrow[0, \log (m+1)]$ be the topological entropy function

$$
h_{\boldsymbol{\sigma}}^{\mathrm{saw}}(\mathbf{p})=h_{\text {top }}\left(S_{\mathbf{p}}\right) .
$$

These functions are continuous by Lemma 5.7 .

Caution: This function $h_{\boldsymbol{\sigma}}^{\text {saw }}: P^{m} \rightarrow[0, \log (m+1)]$ definitely depends on the choice of shape $\boldsymbol{\sigma}$, and also on the fact that we are working with the family of stunted sawtooth maps with specified slope $s>m+1$, rather than some other family. For $m$ odd, the two choices of $\boldsymbol{\sigma}$ are related by a canonical involution of $P^{m}$. However for $m$ even it is important to realize that the two possible choices of shape yield families which are essentially different, and have quite different topological entropy functions.

We will use the notation $\left\{\mathbf{p} \in P^{m} ; h_{\boldsymbol{\sigma}}^{\text {saw }}(\mathbf{p})=h_{0}\right\}$, or briefly $\left\{h_{\boldsymbol{\sigma}}^{\text {saw }}=h_{0}\right\}$, for the isentrope consisting of all $\mathbf{p} \in P^{m}$ with topological entropy $h_{\boldsymbol{\sigma}}^{\text {saw }}(\mathbf{p})$ equal to $h_{0}$. Similarly we sometimes write $\left\{h_{\boldsymbol{\sigma}}^{\text {saw }} \leq h_{0}\right\}$ for the compact subset $\left\{\mathbf{p} \in P^{m} ; h_{\boldsymbol{\sigma}}^{\text {saw }}(\mathbf{p}) \leq h_{0}\right\}$. The object of this section is to prove the following.

Theorem 6.1. For each $h_{0} \in[0, \log (m+1)]$ the isentrope

$$
\left\{\mathbf{p} \in P^{m} ; h_{\boldsymbol{\sigma}}^{\text {saw }}(\mathbf{p})=h_{0}\right\}
$$

is contractible.

The proof will be based on two lemmas. We first construct a partial ordering $\gg$ of the polyhedron $P^{m}$ so that if $\mathbf{p} \gg \mathbf{q}$ then the corresponding stunted sawtooth map $S_{\mathbf{p}}$ has at least as many periodic orbits as does $S_{\mathbf{q}}$. By 4.10 , this will imply that $h_{\boldsymbol{\sigma}}^{\text {saw }}(\mathbf{p}) \geq h_{\boldsymbol{\sigma}}^{\text {saw }}(\mathbf{q})$. Within the interior of $P^{m}$ we can simply say that $\mathbf{p} \gg \mathbf{q}$ if and only if $p_{j} \geq q_{j}$ for all $j$. (As any coordinate $p_{j}$ increases, any periodic orbit which intersects the $j$-th plateau deforms continuously, while any other periodic orbit remains unchanged.) However, to take care of implications on the boundary we must give a more complicated definition. It will be convenient to say that $\mathbf{p}$ contains a level block of length $\ell$ if there are indices $1 \leq i_{0} \leq j_{0} \leq m$ with $\ell=j_{0}-i_{0}+1$ so that

$$
p_{i}+p_{i+1}=1 \quad \text { for } \quad i_{0} \leq i<j_{0} .
$$

This means that the corresponding folding value vector $\mathbf{v}=\mathbf{v}(\mathbf{p})$ satisfies

$$
v_{i_{0}}=v_{i_{0}+1}=\cdots=v_{j_{0}}
$$

so that the corresponding map $S_{\mathbf{p}}$ has $\ell$ consecutive plateaus at the same level. (Here it is convenient to allow the uninteresting case $\ell=1$.) We now define $\gg$ to be the smallest transitive relation on $P^{m}$ which satisfies the following condition: If $\mathbf{p}$ coincides with $\mathbf{q}$ except that $\mathbf{p}$ contains a level block

$$
p, 1-p, p, \ldots, 1-p, p
$$


of odd length $\ell \geq 1$ which is replaced in $\mathbf{q}$ by a corresponding block

$$
q, 1-q, q, \ldots, 1-q, q
$$

of the same length, where $p \geq q$, then $\mathbf{p} \gg \mathbf{q}$.

Lemma 6.2. For each $0 \leq h_{0} \leq \log (m+1)$, the isentrope $\left\{h_{\boldsymbol{\sigma}}^{\text {saw }}=h_{0}\right\}$ is a deformation retract of the region $\left\{h \leq h_{0}\right\}$.

Proof of 6.2. Starting with any point $\hat{\mathbf{p}} \in P^{m}$ we construct a topological entropy increasing path $t \mapsto \mathbf{p}(t) \in P^{m}$ for $0 \leq t \leq 1$ with $\mathbf{p}(0)=\hat{\mathbf{p}}$ and $h_{\boldsymbol{\sigma}}^{\mathrm{saw}}(\mathbf{p}(1))=\log (m+1)$. In fact, let

$$
p_{j}(t)=\min \left(\hat{p}_{j}+t, 1\right) .
$$

Alternatively, this deformation can be described by the differential equation

$$
\frac{d p_{j}}{d t}=\left\{\begin{array}{cl}
+1 & \text { if } p_{j}<1 \\
0 & \text { if } p_{j}=1 .
\end{array}\right.
$$

Clearly the resulting path depends continuously on $\hat{\mathbf{p}}$ and $t$, takes values in $P^{m}$, and satisfies $\mathbf{p}(t) \ll \mathbf{p}\left(t^{\prime}\right)$ whenever $t \leq t^{\prime}$. In particular, it follows that $h_{\boldsymbol{\sigma}}^{\text {saw }}(\mathbf{p}(t)) \leq h_{\boldsymbol{\sigma}}^{\text {saw }}\left(\mathbf{p}\left(t^{\prime}\right)\right)$ whenever $t \leq t^{\prime}$.

It will be convenient to use the norm

$$
\|\mathbf{p}-\mathbf{q}\|=\max _{j}\left|p_{j}-q_{j}\right|
$$

for $\mathbf{p}, \mathbf{q} \in P^{m}$. If $\|\hat{\mathbf{p}}-\hat{\mathbf{q}}\|<\epsilon$, then clearly $\|\mathbf{p}(t)-\mathbf{q}(t)\|<\epsilon$ for $t \in[0,1]$, and furthermore

$$
\mathbf{p}(t+\epsilon) \gg \mathbf{q}(t), \quad \mathbf{q}(t+\epsilon) \gg \mathbf{p}(t) .
$$

Now suppose that $h_{\boldsymbol{\sigma}}^{\text {saw }}(\hat{p}) \leq h_{0}$. Let $t(\hat{\mathbf{p}})$ be the smallest value of $t \in[0,1]$ with $h_{\boldsymbol{\sigma}}^{\text {saw }}(\mathbf{p}(t))=h_{0}$. If $\|\hat{\mathbf{p}}-\hat{\mathbf{q}}\|<\epsilon$, then it follows that $|t(\hat{\mathbf{p}})-t(\hat{\mathbf{q}})|<\epsilon$. Therefore, the homotopy which is defined by

$$
h_{t}(\hat{\mathbf{p}})= \begin{cases}\mathbf{p}(t) & \text { if } t \leq t(\hat{\mathbf{p}}) \\ \mathbf{p}(t(\hat{\mathbf{p}})) & \text { if } t \geq t(\hat{\mathbf{p}})\end{cases}
$$

yields a deformation retraction from $\left\{h_{\boldsymbol{\sigma}}^{\text {saw }} \leq h_{0}\right\}$ onto the isentrope $\left\{h_{\boldsymbol{\sigma}}^{\mathrm{saw}}=h_{0}\right\}$.

Lemma 6.3. The region $\left\{h_{\boldsymbol{\sigma}}^{\text {saw }} \leq h_{0}\right\} \subset P^{m}$ is contractible.

Proof of 6.3. The first step is to construct a topological entropy decreasing deformation $(\hat{\mathbf{p}}, t) \mapsto \mathbf{p}(t)$ which continuously "flattens out the bumps" on the corresponding stunted sawtooth maps $S_{\mathbf{p}(t)}$. This deformation is defined by a differential equation as follows. Let $p, 1-p, p, \ldots$ be any maximal level block contained in $\mathbf{p}$, and let $\ell \geq 1$ be its length. We set

$$
\frac{d p}{d t}=\left\{\begin{array}{cl}
-1 & \text { if } \ell \text { is odd and } p>0, \text { and } \\
0 & \text { if } \ell \text { is even or } p=0 .
\end{array}\right.
$$

(Thus the majority of the entries in a block of odd length move down, but a minority of the entries move up.) Evidently topological entropy decreases monotonically along each path. Furthermore the quantity $p_{1}+\cdots+p_{n}$, which is linearly related to the total 
variation of the map $S_{\mathbf{p}}$, decreases with derivative $\leq-1$ until we reach a stationary state, corresponding to a monotone map with topological entropy equal to zero. This proves that the sub-polyhedron consisting of such stationary states is a deformation retract of the region $\left\{h_{\boldsymbol{\sigma}}^{\text {saw }} \leq h_{0}\right\}$. In the case $m$ odd, this sub-polyhedron consists of a single point, corresponding to the constant map. This completes the proof of 6.3 for $m$ odd.

For $m=2 k$, this sub-polyhedron is a $k$-simplex, consisting of all

$$
\left(p_{1}, 1-p_{1}, p_{3}, 1-p_{3}, \ldots, p_{m-1}, 1-p_{m-1}\right)
$$

with $p_{1} \leq p_{3} \leq \cdots \leq p_{m-1}$. Since this simplex is itself contractible, this completes the proof of 6.3 .

Theorem 6.1 now follows, since a retract of a contractible space is clearly contractible (if $F_{\lambda}$ is a contraction of the total space, $r$ a retraction, and $e$ the embedding of the retract in the total space, then $r \circ F_{\lambda} \circ e$ is a contraction of the retract).

\section{$\S$ 7. Bones in $P^{2}$.}

The stunted sawtooth families are well understood, but the polynomial families of the same shape are poorly understood beyond the unimodal case. Our analysis of the relationship between these two families in the cubic case will rely on the study of parameter points for which at least one of the two critical orbits is periodic.

We will need a precise terminology for describing periodic orbits. As in $\S 1$, let o be a cyclic permutation of the integers $\{1,2, \ldots, q\}$. By definition, a periodic orbit $\mathcal{O}=$ $\left\{x_{1}, \ldots, x_{q}\right\}$ of an interval map $f$ is said to have order type $\mathbf{o}$ if $f$ maps each $x_{i}$ to $x_{\mathbf{o}(i)}$, where $x_{1}<x_{2}<\cdots<x_{q}$. We will sometimes use the notation $\mathbf{o}=\left(i_{1} i_{2} \ldots i_{p}\right)$ for the permutation which satisfies $\mathbf{o}\left(i_{j}\right)=i_{j+1}$, so that

$$
f\left(x_{i_{j}}\right)=x_{i_{j+1}} .
$$

Here the subscripts $j$ are to be taken modulo $p$.

Definition. A cyclic permutation o will be called m-modal of shape $\boldsymbol{\sigma}$ if there exists an $m$-modal map of shape $\boldsymbol{\sigma}$ which has a periodic orbit with order type $\mathbf{o}$. It will be called strictly $m$-modal of shape $\boldsymbol{\sigma}$ if there exists such a map with all $m$ of its critical points on this orbit. Equivalently, this means that there are integers

$$
1 \leq \gamma_{1}<\gamma_{2}<\cdots<\gamma_{m} \leq q,
$$

where $x_{\gamma_{1}}<\cdots<x_{\gamma_{m}}$ are to be the critical points, with the following property. Setting $\gamma_{0}=1, \gamma_{m+1}=q$, the restriction of the permutation $\mathbf{o}$ to the integers in each interval $\left[\gamma_{i}, \gamma_{i+1}\right]$ must be either monotone increasing or monotone decreasing according as $\sigma_{i}$ equals +1 or -1 . Of course this condition implies that the period $q$ must satisfy $q \geq m$. Given any periodic orbit of this order type, for a map of shape $\boldsymbol{\sigma}$, note that the address of the orbit point $x_{i}$ is necessarily given by

$$
A\left(x_{i}\right)=\left\{\begin{array}{lll}
I_{0} & \text { if } & i<\gamma_{1} \\
I_{j} & \text { if } & \gamma_{j}<i<\gamma_{j+1} \\
I_{m} & \text { if } & \gamma_{m}<i .
\end{array}\right.
$$


However, when $i$ is precisely equal to $\gamma_{j}$ the address $A\left(x_{i}\right)$ is not uniquely determined: it can be $C_{j}$ but it can also be either of the adjacent intervals $I_{j-1}$ or $I_{j}$. Thus altogether there are $3^{m}$ distinct possibilities. It follows that there are $3^{m}$ different possibilities for the kneading type of the periodic orbit (that is, the itinerary of a representative point, which is well defined up to a shift). Compare the proof of 7.1 below.

It is not difficult to check that a bimodal order type of shape $(+-+)$ is strictly $(+-+)-$ bimodal if and only if its period satisfies $q \geq 2$. On the other hand, a bimodal order type of shape $(-+-)$ is strictly $(-+-)$-bimodal if and only if $q \geq 3$.
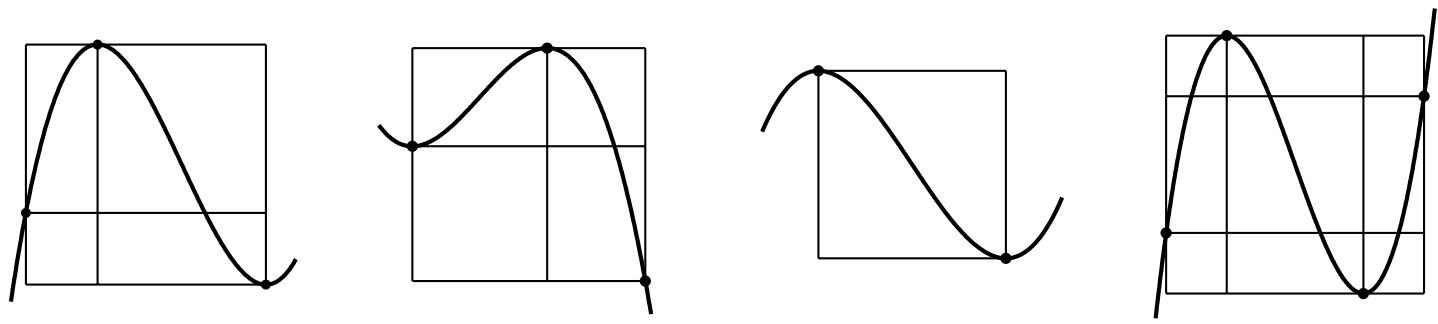

Figure 10. The period 3 order type $\mathbf{o}=(123)$ is strictly bimodal of shape either $(+-+)$ or $(-+-)$, but the period 2 order type is strictly bimodal only of shape $(+-+)$, and the period 4 order type $\mathbf{o}=(1243)$ is bimodal only of shape $(+-+)$.

In order to relate two families of the same shape in the bimodal case, we introduce some terminology from MacKay and Tresser [MaT1]. As in $\S 3$, consider a family of bimodal maps of some fixed shape $\boldsymbol{\sigma}$, parameterized by the triangle $P^{2}$.

Definition. By a bone in the parameter space $P^{2}$ we mean the compact set consisting of all parameter values for which a specified critical point has periodic orbit with specified order type. More precisely, the left bone $B_{-}(\mathbf{o})$ is the set of parameter values for which the left hand critical point is periodic with order type $\mathbf{o}$. The dual right bone $B_{+}(\mathbf{o})$ is the set of parameter values for which the right critical point is periodic with this same order type. Note that two left bones, or two right bones, are disjoint, almost by definition. These definitions make sense either for the stunted sawtooth family or for the cubic family. We will insert the superscript "saw" respectively "cub" in order to distinguish these two cases. Similarly, we will use the notation $P^{\text {saw }}$ or $P^{\text {cub }}$ for the parameter triangle $P^{2}$ when we want to emphasize that it is being considered as the parameter space for stunted sawtooth maps or for cubic maps.

In discussing the parameter triangle $P^{2}$, we will refer to the vertex $\mathbf{p}=(1,1)$, corresponding to a map of entropy $\log 3$, as the top vertex. The opposite edge, corresponding to monotone maps, with entropy zero, will be called the bottom edge of $P^{2}$.

In either the stunted sawtooth or the cubic family of shape $\boldsymbol{\sigma}$, it is not difficult to check that a bone $B_{ \pm}(\mathbf{o})$ is non-vacuous if and only if its order type $\mathbf{o}$ is bimodal of shape $\boldsymbol{\sigma}$. We will concentrate on order types which are strictly $\boldsymbol{\sigma}$-bimodal. In the three exceptional cases where $\mathbf{o}$ is not strictly bimodal of this shape (that is for period two with shape 
$(-+-)$ or for period one with either shape), the bones behave rather differently. For example, in these cases only, the corresponding bones intersect the bottom edge of the triangle $P^{2}$ (see Figure 14). These exceptional cases will play only a minor role in our argument. (Compare $\S 8$.)
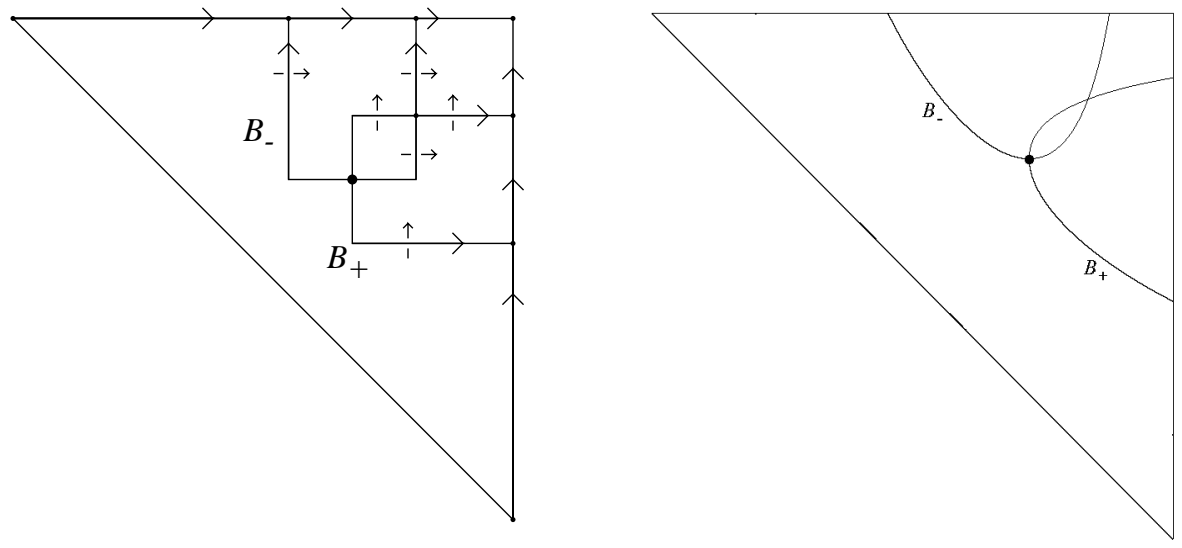

Figure 11. The period 2 bones for the stunted sawtooth family of shape $(+-+)$ on the left, and for the corresponding cubic family on the right, with the primary intersection points emphasized. Arrows point in the direction of increasing dynamic complexity. (The pictures for either shape $\sigma$ and for any strictly bimodal order type of this shape would look qualitatively the same.)

Suppose then that $\mathbf{o}$ is strictly bimodal of shape $\boldsymbol{\sigma}$. We consider first the stunted sawtooth case, which is much easier to analyze.

Lemma 7.1. For either bimodal shape $\boldsymbol{\sigma}$ and for each strictly $\boldsymbol{\sigma}$-bimodal order type $\mathbf{o}$ :

(i) Each bone $B_{ \pm}^{\mathrm{saw}}(\mathbf{o})$ is a simple arc with both endpoints on a common vertical or horizontal edge of the triangle $P^{2}=P^{\text {saw }}$, and is made up out of three straight line segments which are alternately horizontal and vertical.

(ii) The dual bones $B_{-}^{\text {saw }}(\mathbf{o})$ and $B_{+}^{\text {saw }}(\mathbf{o})$ intersect transversally in exactly two points. The intersection at the midpoints of their middle line segments will be called their primary intersection point. It corresponds to the unique map $S_{\mathbf{p}}$ for which both critical points lie on a common periodic orbit of order type o. There is also a secondary intersection point, higher in $P^{\text {saw }}$, corresponding to the unique map $S_{\mathbf{p}}$ which has two disjoint critical orbits, both periodic with the same order type o.

(iii) This pair of dual bones cuts the triangle $P^{\text {saw }}$ into five regions. There is a large region which contains the entire bottom edge of $P^{\text {saw }}$ and contains the primary intersection point in its boundary. Adjoining this are two side regions, each touching just one edge of $P^{\text {saw }}$. Next there is a central region, 
which is disjoint from all other bones so that the topological entropy takes a constant value (equal to the logarithm of an algebraic unit) throughout. Finally, there is a top region whose boundary contains both the secondary intersection point and the top vertex of $P^{\text {saw }}$.

(iv) For $\mathbf{o} \neq \mathbf{o}^{\prime}$, the bones $B_{-}^{\text {saw }}(\mathbf{o})$ and $B_{+}^{\text {saw }}\left(\mathbf{o}^{\prime}\right)$ intersect transversally in either 0, 2, or 4 points. Each of these intersection points corresponds to a map with two disjoint periodic critical orbits. Again the entropy is the logarithm of an algebraic unit.

Proof of (i), (ii) and (iv). Since the order type $\mathbf{o}$ is strictly bimodal of shape $\boldsymbol{\sigma}$, it follows easily from 5.3 that there exists a point $\mathbf{p}^{0}=\left(p_{1}^{0}, p_{2}^{0}\right) \in P^{\text {saw }}$ so that both critical points of the map $S_{\mathbf{p}^{0}}$ lie in a common period $q$ orbit with order type $\mathbf{o}$. (The argument will show that this point $\mathbf{p}^{0}$ is unique. Compare 8.1 below.) Evidently there is a unique $0<n<q$ so that the iterate $S_{\mathbf{p}^{0}}^{\circ n}$ maps the critical point $\widehat{C}_{1}$ to $\widehat{C}_{2}$ while $S_{\mathbf{p}^{0}}^{\circ(q-n)}$ maps $\widehat{C}_{2}$ to $\widehat{C}_{1}$. The kneading type $\mathcal{I}^{0}$ of this periodic orbit, that is the itinerary of a representative point (well defined up to a shift), is periodic of period $q$, and contains each of the critical point symbols $C_{1}$ and $C_{2}$ exactly once in each period. For an arbitrary periodic orbit of the same order type, as noted above, there are $3^{m}=9$ different possibilities for the kneading type: If we replace $\left(p_{1}^{0}, p_{1}^{0}\right)$ by some nearby point $\left(p_{1}, p_{2}\right)$, then there will still be a unique periodic orbit which intersects both plateaus. However, depending on the sign of $p_{2}-p_{2}^{0}$ and of $p_{1}-p_{1}^{0}$, we can replace the symbol $C_{1}$ in the kneading type $\mathcal{I}^{0}$ by either of the two adjacent symbols $I_{0}$ or $I_{1}$, and we can replace $C_{2}$ by either $I_{1}$ or $I_{2}$. However, no other replacements are possible. In particular, for any orbit of order type o for a bimodal map of shape $\boldsymbol{\sigma}$, the remaining $q-2$ symbols must remain unchanged.

To fix our ideas, consider a left bone $B_{-}(\mathbf{o})$. For $\mathbf{p} \in B_{-}(\mathbf{o})$ the left critical point $\widehat{C}_{1}$ is periodic. Evidently each plateau of a stunted sawtooth map can contain at most one periodic point, and for $\mathbf{p} \in B_{-}(\mathbf{o})$ it is easy to check that only the point $S_{\mathbf{p}}^{\circ n}\left(\widehat{C}_{1}\right)$ in the orbit of $\widehat{C}_{1}$ can lie in the right hand plateau. We divide the discussion into three cases, corresponding to the three line segments which make up the bone $B_{-}(\mathbf{o})$.

Case 1. If $S_{\mathbf{p}}^{\circ n}\left(\widehat{C}_{1}\right)=S_{\mathbf{p}}^{\circ(n-1)}\left(v_{1}\right)$ does lie in the right hand plateau, then the iterate $S_{\mathbf{p}}^{\circ(n-q-1)}$ must map the critical value $v_{2}=S_{\mathbf{p}}\left(\widehat{C}_{2}\right)$ to $\widehat{C}_{1}$. Since the partial itinerary from $v_{2}$ to $\widehat{C}_{1}$ is uniquely determined by the order type, this yields a linear equation which we can solve for $v_{2}$, and hence for $p_{2}=p_{2}^{0}$.

Cases 2, 3. On the other hand, if $S_{\mathbf{p}}^{\circ n}\left(\widehat{C}_{1}\right)$ lies either to the left or to the right of the right hand plateau, then the entire partial itinerary from $v_{1}$ back to $\widehat{C}_{1}$ is uniquely determined. In either of these two cases, this yields a linear equation which we can solve for $v_{1}$.

Alternatively, we can describe these three cases as follows. Suppose that we fix $p_{2}=p_{2}^{0}$ and vary $p_{1}$ throughout a neighborhood of $p_{1}^{0}$. Then the size of the right hand plateau remains fixed, and there will be a largest interval $\left[p_{1}^{-}, p_{1}^{+}\right]$, symmetric about $p_{1}^{0}$, so that, for $p_{1}$ in this interval, the image $S_{\mathbf{p}}^{\circ(n-1)}\left(v_{1}\right)$ lies in the right hand plateau. For the 
extreme values $p_{1}=p_{1}^{ \pm}$, this image will lie at either end of the plateau. Now fix $p_{1}=p_{1}^{ \pm}$ and let $p_{2}$ vary over the interval $\left[p_{2}^{0}, 1\right]$. As $p_{2}$ increases, the right hand plateau will move up or down (depending on $\boldsymbol{\sigma}$ ), shrinking in length, but the orbit of $\widehat{C}_{1}$ will remain fixed, missing the right hand plateau completely when $p_{2}>p_{2}^{0}$. It follows that $B^{-}(\mathbf{o})$ is the union of three line segments:

$$
B_{-}(\mathbf{o})=p_{1}^{-} \times\left[p_{2}^{0}, 1\right] \cup\left[p_{1}^{-}, p_{1}^{+}\right] \times p_{2}^{0} \cup p_{1}^{+} \times\left[p_{2}^{0}, 1\right] .
$$

The discussion of right bones in completely analogous. This proves Part (i); and Parts (ii), (iv) follow easily.

Proof of (iii). It is easy to see that a pair of dual bones partitions $P^{\text {saw }}$ into five regions. To prove that the central region is disjoint from all other bones, let us follow the left bone $B_{-}(\mathbf{o})$ from the primary intersection point $\mathbf{p}^{0}$ to the secondary intersection point. For each point $\mathbf{p}$ in this path, the image $C^{\prime}(\mathbf{p})=S_{\mathbf{p}}^{\circ n}\left(\widehat{C}_{1}\right)$ is periodic, belonging to the orbit of $\widehat{C}_{1}$. Let $J_{\mathbf{p}}$ be the interval with endpoints $\widehat{C}_{2}$ and $C^{\prime}(\mathbf{p})$. For $\mathbf{p}=\mathbf{p}^{0}$ this interval degenerates to a point, and for $\mathbf{p}$ in a large neighborhood of $\mathbf{p}^{0}$ the iterate $S_{\mathbf{p}}^{\circ q}$ maps all of $J_{\mathbf{p}}$ to the endpoint $C^{\prime}(\mathbf{p})$. However, as $\mathbf{p}$ moves towards the secondary intersection point, eventually its image under $S_{\mathbf{p}}^{\circ q}$ will become bigger. However, the restriction of $S_{p}^{\circ q}$ to $J_{\mathbf{p}}$ will remain monotone, and its image will remain a proper subset of $J_{\mathbf{p}}$ until $\mathbf{p}$ reaches the secondary intersection point, at which time $S_{\mathbf{p}}^{\circ q}\left(\widehat{C}_{2}\right)$ will equal $\widehat{C}_{2}$, so that $J_{\mathbf{p}}$ maps onto itself with both endpoints fixed. Until $\mathbf{p}$ reaches this point, clearly $\widehat{C}_{2}$ will not be periodic, so no other bone can cross through to the central region.

The statement that topological entropy is constant in any region without bones follows easily from 4.11. (Compare 9.1 below.) Such a region necessarily contains hyperbolic points by 5.9. Hence this constant value must be the logarithm of an algebraic integer by 4.3. This proves (iii), and completes the proof of 7.1.

The statement corresponding to Lemma 7.1 for the cubic family is much more difficult. Here is some preliminary information. Again let $\mathbf{o}$ be strictly bimodal of shape $\boldsymbol{\sigma}$.

Lemma 7.2. Both bones $B_{ \pm}^{\mathrm{cub}}(\mathbf{o})$ are smooth 1-dimensional manifolds with boundary. Further, the boundary of $B_{-}^{\mathrm{cub}}(\mathbf{o})$ is equal to the transversal intersection of this bone with the horizontal (top) edge of the triangle $P^{\text {cub }}$, while the boundary of $B_{+}^{\mathrm{cub}}(\mathbf{o})$ is equal to the transversal intersection of this bone with the vertical (right hand) edge of $P^{\mathrm{cub}}$. Any intersection $B_{-}^{\mathrm{cub}}(\mathbf{o}) \cap B_{+}^{\mathrm{cub}}\left(\mathbf{o}^{\prime}\right)$ between two such bones is also transverse.

(If we make use of Heckman's Theorem that there are no bone-loops, then we have the much sharper statement that each bone is a connected simple arc.)

The proof of 7.2 begins as follows. First consider the corresponding statement for the family of complex maps $z \mapsto z^{3}-3 a^{2} z+b$, with critical points $\pm a$. It is proved in [M3] that the locus $\mathcal{S}_{ \pm}(p)$ of points for which $\pm a$ has period $p$ is a smooth complex curve. Furthermore, for each $p$ and $q$ the curves $\mathcal{S}_{+}(p)$ and $\mathcal{S}_{-}(q)$ intersect transversally. (See [M2].) In fact, $\mathcal{S}_{+}(p)$ has transverse intersection with any curve consisting of points for which the other critical point $-a$ is preperiodic. The proofs make essential use of quasi- 
conformal surgery. Compare [St], where analogous results for quadratic rational maps are proved by similar methods. Alternatively, these statements have been proved by quite different methods by Epstein [E].

Restricting to the real $(a, b)$-plane, we obtain a corresponding statement for real cubic maps of shape $(+-+)$. In the family of real maps

$$
x \mapsto x^{3}-3 a^{2} x+b,
$$

the locus of pairs $(a, b)$ for which a (or $-a)$ is periodic of period $p$ forms a smooth 1dimensional manifold without boundary. A complex linear change of coordinates yields the corresponding statement for the family of maps

$$
x \mapsto-\left(x^{3}-3 a^{2} x+b\right),
$$

of shape $(-+-)$.

In order to relate this to the family of cubic maps $f_{\mathbf{p}}$, we note that each $f_{\mathbf{p}}$ is positively affinely conjugate to a unique map in the normal form $\left(17^{ \pm}\right)$with $a \geq 0$. That is, for each $\mathbf{p}$ there is a unique affine map $L(x)=c x+d$ with $c>0$ so that $L \circ f_{\mathbf{p}} \circ L^{-1}$ has the required form. It follows that there is a well defined continuous mapping $\phi: P^{\text {cub }} \rightarrow \mathbb{R}^{2}$ which associates to each $\mathbf{p} \in P^{\text {cub }}$ the associated pair $\phi(\mathbf{p})=(a, b)$ in the half-plane $a \geq 0$. Evidently the pre-image of the curve $\mathcal{S}_{ \pm}(p)$ under $\phi$ is the union of all bones $B_{ \pm}^{\text {cub }}(\mathbf{o})$ of period $p$. If $\phi$ were a diffeomorphism, then it would follow immediately that each bone is a smooth 1-manifold, with boundary points precisely at the intersections with the horizontal or vertical part of the boundary of $P^{\text {cub }}$. In fact, the situation is somewhat more complicated, and can be described as follows. (Compare [DGMT, Figures 8,9.)

Lemma 7.3. Let $P^{\mathrm{cub}}$ be the parameter triangle for cubic maps of specified shape $\boldsymbol{\sigma}$, and let $U \subset P^{\text {cub }}$ be the open subset consisting of those parameter values $\mathbf{p}$ such that the boundary $\partial I$ is strictly repelling for the associated cubic map $f_{\mathbf{p}}: I \rightarrow I$. Then every bone of strictly bimodal order type in $P^{\text {cub }}$ is contained in this open set $U$. Furthermore, the map $\phi: P^{\text {cub }} \rightarrow \mathbb{R}^{2}$ embeds $U$ diffeomorphically into $\mathbb{R}^{2}$.

Clearly Lemma 7.2 will follow from 7.3, together with the discussion above.

Proof of 7.3. If $\mathbf{p}$ belongs to the complement $P^{\text {cub }} \backslash U$, then some boundary orbit of period one or two for $f_{\mathbf{p}}$ has multiplier in the interval $[0,1]$. Since $f_{\mathbf{p}}$ has negative Schwarzian derivative, this implies that there is at least one critical point in the immediate basin, and this clearly implies that the interval between the two critical points is also contained in this immediate basin. Thus there cannot be any periodic critical orbit, unless one and hence both critical points are actually contained in the boundary $\partial I$. This occurs only if $\mathbf{p}$ is one of the two lower corner points of the parameter triangle. Evidently the corresponding order type $\mathbf{o}$ is not strictly bimodal. (These zero entropy corner points are quite anomalous, since each one represents an isolated point of the associated bone of period one or two.)

For any $\mathbf{p} \in U$ the boundary periodic orbit (in the $(-+-)$ case) or orbits (in the $(+-+)$ case) are strictly repelling, and hence vary smoothly under smooth deformation of the polynomial. It follows that there is a smooth local inverse function from the image 
$\phi(U) \subset \mathbb{R}^{2}$ back to $U$. This inverse function is single valued, since otherwise we would have two different intervals, say $I$ and $I^{\prime}$, both containing the two critical points, so that the same polynomial function $f_{\mathbf{p}}$ maps each of these intervals into itself with strictly repelling boundary. Then any component of $I \backslash I^{\prime}$ would map diffeomorphically into itself under $f_{\mathbf{p}}$ or $f_{\mathbf{p}} \circ f_{\mathbf{p}}$, with both boundary points repelling, which is impossible for a map of negative Schwarzian. This completes the proof of 7.3 and 7.2 .
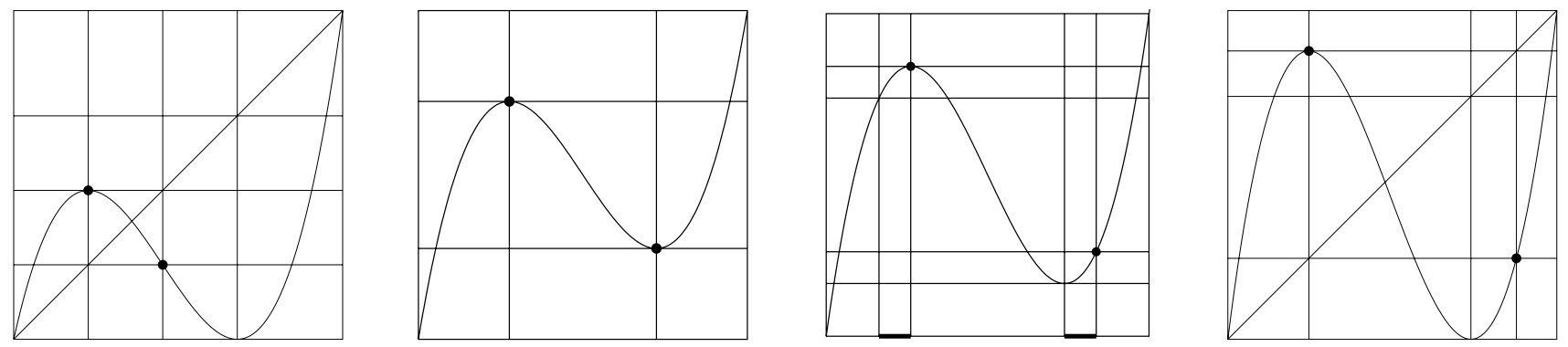

Figure 12. Graphs of cubic maps of shape $(+-+)$ representing successive points along the left bone of period two. Illustrated are the "lower" endpoint of this bone, the primary intersection point where dynamic complexity is minimized, the secondary intersection point, and the "upper" endpoint. In the third graph there are three different period two orbits, all contained in the emphasized intervals, and these period two orbits all persist to the right hand graph, which has the largest negative orbit complexity.

Remark 7.4. There is a fundamental relationship between bones and negative orbit complexity. (Compare 9.1.) In fact, as we traverse a path in the parameter triangle, the number of fixed points of negative type for the $n$-th iterate can clearly change only as we cross a bone of period dividing $n$. More precisely, as we cross a period $p$ bone in the 'positive' direction, as indicated in Figure 11, it turns out that exactly one new periodic orbit of negative type is created, with period equal to $p$. This fact can be used to count the number of period $p$ bones. In the $(+-+)$-case, note that the number of fixed points of negative type for the $n$-th iterate increases from zero, along the bottom edge of the parameter triangle, to a maximum of $\left(3^{n}-1\right) / 2$ for the map, say $S_{(1,1)}$, corresponding to the top (high entropy) vertex of the triangle. It follows easily that \#Bones $(p)$, the number of period $p$ bones in the parameter triangle (suitably interpreted when $p=1$ ), is precisely equal to the number of period $p$ orbits of negative type for the map $S_{(1,1)}$. From this, we derive the following formula, which can be used to compute \#Bones $(p)$.

$$
\left(3^{n}-1\right) / 2=\sum_{p \mid n \text { with } n / p \text { odd }} p \cdot \# \operatorname{Bones}(p)
$$

For example:

$$
\begin{array}{cccccccccc}
p= & 1 & 2 & 3 & 4 & 5 & 6 & 7 & 8 & 9 \\
\left(3^{p}-1\right) / 2= & 1 & 4 & 13 & 40 & 121 & 364 & 1093 & 3280 & 9841 \\
\text { \#Bones }(p)= & 1 & 2 & 4 & 10 & 24 & 60 & 156 & 410 & 1092 .
\end{array}
$$


Note that \#Bones $(p)$ is even for $p>1$, since the bones occur in dual pairs. The discussion for the $(-+-)$-case is slightly different, but the number \#Bones $(p)$ remains the same.

Remark 7.5. Evidently the intersection points of bones are precisely those points in parameter space for which both critical points are periodic. We will see in $\S 8$ that there is a one-to-one correspondence, preserving kneading data, between these intersection points for the stunted sawtooth case and for the cubic case. Alternatively, this statement would follow easily from a form of Thurston's Theorem, as stated in B.3 and B.4 of Appendix B. In general, for the cubic map corresponding to any intersection of bones, the two critical points will belong to disjoint periodic orbits. The only exception is for the primary intersection point between two dual bones. In this exceptional case, the two critical points belong to a common orbit. (See Figure 11 for the picture in $P^{2}$, and see Figure 10 for graphs of some cubic maps corresponding to primary intersections between dual bones.)

Similarly, the endpoints of strictly $\boldsymbol{\sigma}$-bimodal bones correspond to maps for which one critical point is periodic while the other is preperiodic, mapping directly to a boundary fixed point or period two orbit. Again there is a canonical one-to-one correspondence between such endpoints for the stunted sawtooth and cubic families. In particular, it follows that each cubic bone has exactly two endpoints. (See Figure 12 for graphs of several maps corresponding to parameter values along a left bone, including the two endpoints.)

\section{$\S 8$. Monotonicity, Intersections of Bones, and the $n$-Skeleton.}

In the quadratic case, it is known that the number of period $q$ points for the interval map $Q_{v}(x)=4 v x(1-x)$ increases monotonically as the folding value parameter $v \in[0,1]$ increases. Similarly the kneading sequence $\mathcal{K}_{1}\left(Q_{v}\right)$ increases monotonically with $v$. Proofs of this result have been given by Sullivan, ${ }^{8}$ Douady and Hubbard, and by Milnor and Thurston. Compare [DH2, no VI], [MTh], [D], as well as [MvS2].

We can adapt similar techniques to study special one parameter families of cubic maps. (Compare $[\mathrm{NN}]$.$) [KN].) However, instead of working with the full kneading data, it will$ suffice for our purposes to work with the "negative orbit complexity"

$$
\mathcal{N}(f)=\left(\operatorname{Neg}\left(f^{\circ 1}\right), \operatorname{Neg}\left(f^{\circ 2}\right), \ldots\right)
$$

of 4.13. In fact, we will establish monotonicity of $\mathcal{N}(f)$ in a collection of one parameter families large enough to give us a good picture of the full two parameter family, making use of Heckman's Theorem that there are no bone-loops.

By definition, a polynomial map is post-critically finite if the orbit of every critical point is periodic or eventually periodic. Similarly, we will say that a piecewise monotone map has finite folding point orbits if the orbit of every folding point is periodic or eventually periodic. (If $f: I \rightarrow I$ is piecewise strictly monotone and boundary anchored, then a completely equivalent condition is that $f$ be a "Markov map" in the sense that there exist

\footnotetext{
${ }^{8}$ As far as we know, Sullivan did not actually publish a proof. However he communicated one orally at an early date, and conversations with Sullivan led to the Milnor-Thurston proof.
} 
a subdivision of $I$ into finitely many subintervals, each of which maps homeomorphically onto some union of these subintervals.)

Thurston Uniqueness Theorem for Real Polynomial Maps. A postcritically finite real polynomial map of degree $m+1$ with $m$ distinct real critical points is uniquely determined, up to positive affine conjugation, by its kneading data.

See Appendix B, which augments this to a full existence and uniqueness statement, and gives some indication of the proof. There is an analogous statement for the stunted sawtooth family, as follows. As usual, we assume that the slope $s>m+1$ has been fixed.

Lemma 8.1. Given any piecewise strictly monotone m-modal map such that each folding point has finite forward orbit, there exists one and only one stunted sawtooth map of the same shape with the same kneading data.

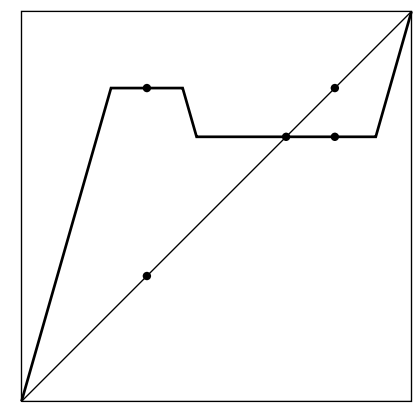

Caution. Here it is essential that the kneading data comes from a piecewise strictly monotone map. The following figure shows an example to illustrate this. Recall that the "folding points" of a stunted sawtooth map are defined to be the midpoints $\widehat{C}_{i}$ of the plateaus. Let $S_{\mathbf{p}}$ be a stunted sawtooth map as illustrated, of shape $(+-+)$ with folding values $v_{1}=\widehat{C}_{2}+\epsilon>v_{2}=\widehat{C}_{2}$. If $\epsilon>0$ is sufficiently small, then this map is post-critically finite, with kneading data $\mathcal{K}_{1}=\left(I_{2}, \bar{C}_{2}\right), \mathcal{K}_{2}=\bar{C}_{2}$, where the overline stands for infinite repetition. Thus there is an entire 1-parameter family of stunted sawtooth maps with the same post-critically finite kneading data. Yet no polynomial map, and more generally no piecewise strictly monotone map, can have this kneading data, which requires that an entire interval in $I_{2}$ must map to the second folding point. (Compare Appendix B.)

In fact there is a simple criterion which guarantees that the kneading data for some given post-critically finite map $f$ can be realized by a map which is piecewise strictly monotone. Let $\left\{y_{1}, \ldots, y_{n}\right\}$ be the union of the forward orbits of the folding points, where $y_{1}<\cdots<y_{n}$. If $f\left(y_{i}\right) \neq f\left(y_{i+1}\right)$ for $1 \leq i<n$, then interpolating linearly between the $y_{i}$, and extending suitably over the rest of the interval $[a, b]$, we easily obtain a piecewise strictly monotone map with the same kneading data.

Proof of 8.1. Existence follows from 5.3. To prove uniqueness of the resulting stunted sawtooth map $S_{\mathbf{p}}$, note that the orbit of a folding point of $S_{\mathbf{p}}$ can never land in a plateau, except at its midpoint $\widehat{C}_{j}$. For otherwise, any map with the same kneading data would 
have an interval of points eventually mapping to the same point, which would contradict the hypothesis. Therefore, orbit points are uniquely determined by their itineraries, hence $S_{\mathbf{p}}$ itself is uniquely determined.

We now proceed to apply these results to the study of monotonicity along special curves. As a first example, consider the top edge $p_{2}=1$ of the parameter triangle for either cubic or stunted sawtooth maps of fixed shape $\boldsymbol{\sigma}$. This edge corresponds to the set of maps $f: I \rightarrow I$ (in either family) such that the second critical value $v_{2}$ is an endpoint of the interval $I$, and hence is either a fixed point, in the $(+-+)$ case, or a period two point, in the $(-+-)$ case. Thus the kneading sequence $\mathcal{K}_{2}$ remains constant as we traverse this edge, and only $\mathcal{K}_{1}$ varies.

Lemma 8.2 In either the cubic or the stunted sawtooth family of specified shape $\boldsymbol{\sigma}$, as we traverse the top edge $p_{2}=1$ of the parameter triangle in the direction of increasing $p_{1}$, the negative orbit complexity $\mathcal{N}$ increases monotonically. More precisely, $\mathcal{N}$ increases whenever we cross the endpoint of a left bone, and remains constant otherwise. Furthermore these crossing points occur in the same order in the two families: To each crossing point in one family, there corresponds one and only one crossing point in the other family with the same kneading data; where the crossing point $f$ (in either family) occurs before $f^{\prime}$ (in the same family) if and only if $\mathbf{K}(f) \ll \mathbf{K}\left(f^{\prime}\right)$.
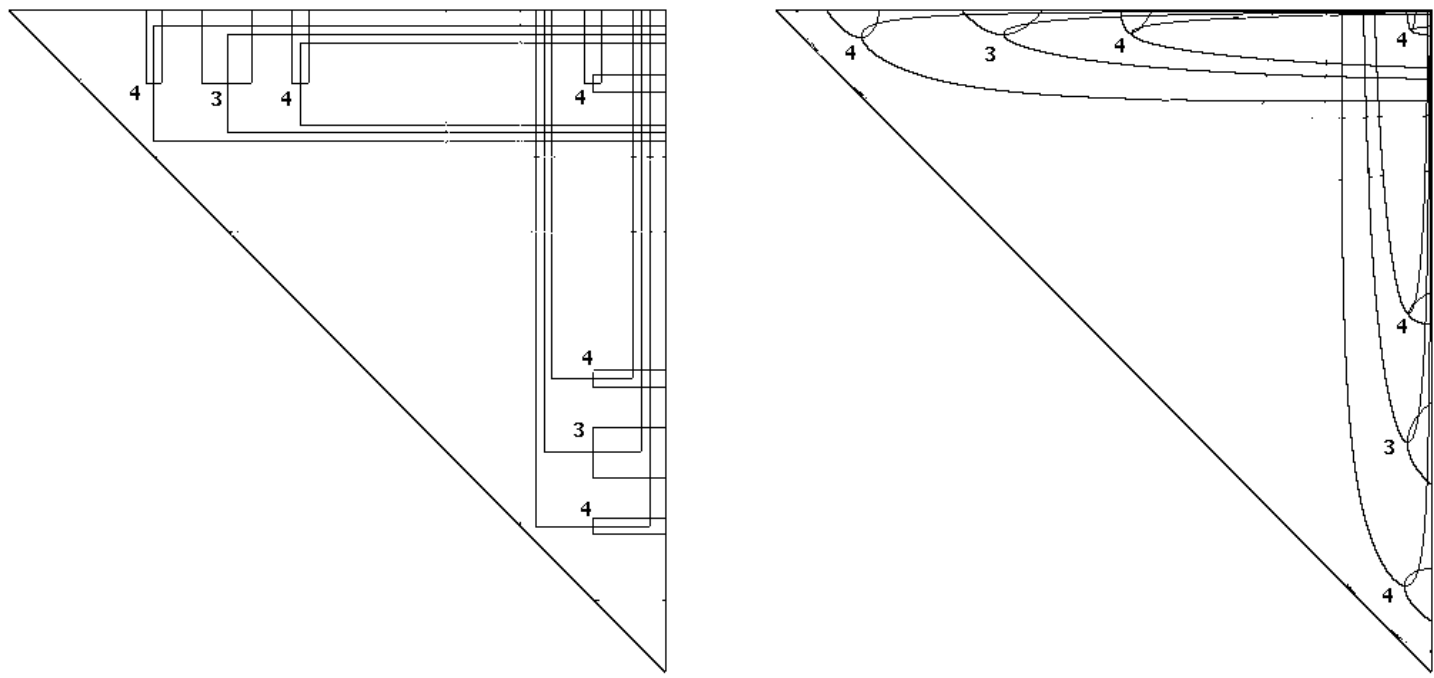

Figure 13. Bones of period 3 and 4 for the stunted sawtooth family of shape $(-+-)$ on the left and for the cubic family of shape $(-+-)$ on the right. Periods are indicated near the primary intersection points.

(Compare 4.5 for this last notation.) It follows that topological entropy also increases monotonically as we traverse this edge. Of course there is a completely analogous statement as we traverse the right hand edge $p_{1}=1$ in the direction of increasing $p_{2}$. It must be 
emphasized that this lemma compares cubic and stunted sawtooth maps for some fixed shape $\boldsymbol{\sigma}$. If we switch shape, then the combinatorics becomes completely different.

Proof of 8.2. At the left endpoint $\mathbf{p}=(0,1)$ of this edge, either in the cubic or the stunted sawtooth family, the associated map is monotone, with no periodic orbit of negative type other than a single fixed point of negative type in the $(-+-)$ case. On the other hand, at the right endpoint $\mathbf{p}=(1,1)$, we have a bimodal map of maximal entropy, with three non-overlapping subintervals each of which maps onto the entire interval $I$. For such a maximal entropy map $f$ there are $3^{n}$ fixed points of $f^{\circ n}$ with distinct itineraries, and approximately half of these have negative type. Thus, as $p_{1}$ increases, many periodic orbits of negative type must be created. However, an orbit of negative type is a relatively robust object which can only be created or destroyed at a parameter value for which one of the orbit points becomes a folding point. Here only the left folding point $c_{1}$ can be the one in question, since the condition $p_{2}=1$ guarantees that the orbit of the right folding point $c_{2}$ never returns to $c_{2}$.

More precisely, we claim the following: In either family, as $p_{1}$ increases through a value such that the orbit of $c_{1}$ is periodic, a periodic orbit of negative type is created. This orbit persists until we reach the right hand endpoint with $\mathbf{p}=(1,1)$, and all periodic orbits of negative type (with the exception of a single fixed point of negative type in the $(-+-)$ case) arise in this way.

There is only one step we have to be careful about: We must be sure that an orbit of negative type, once created, cannot disappear again as $p_{1}$ increases. But this would imply that there were two different parameter values along this edge for which the left folding point is periodic, with the same itinerary in both cases. (It is not difficult to check that there is only one point of such an orbit which can merge with the left critical point.) This is impossible by Thurston's Theorem in the cubic case, ${ }^{9}$ or by 8.1 in the stunted sawtooth case.

Thus the invariant $\mathcal{N}$ increases monotonically as we follow the edge, and increases as we cross each bone. But according to $4.9, \mathcal{N}$ is determined by the kneading data. Hence bone-ends with the same kneading data, in the cubic and stunted sawtooth families, must occur in the same order, as asserted.

Now let us restrict attention to bones which are strictly $\boldsymbol{\sigma}$-bimodal. Thus we exclude only a few low period bones which are atypical (as illustrated in Figure 14).

Lemma 8.3. In the cubic family, just as in the stunted sawtooth family, each strictly $\boldsymbol{\sigma}$-bimodal left bone $B_{-}(\mathbf{o})$ intersects the corresponding right bone $B_{+}(\mathbf{o})$ in exactly two points, and just one of these (the "primary intersection point") has the property that both folding points belong to a common periodic orbit.

Proof. If there were no intersection point, then we could find a path from the maximal

\footnotetext{
${ }^{9} \mathrm{As}$ in the proof of 7.2 , we must be careful since the map from parameter triangle to positive affine conjugacy classes of cubic polynomials is not one-to-one, but rather folds over two corners of the triangle. However by 7.3 , there are no periodic critical orbits for parameters in the folded over region.
} 
entropy vertex $\mathbf{p}=(1,1)$ of the parameter triangle which avoids both $B_{-}(\mathbf{o})$ and $B_{+}(\mathbf{o})$ and yet leads to the opposite edge. This is impossible, since any negative orbit with strictly bimodal order type o must disappear before we reach this lower edge, which parametrizes only monotone maps. Hence there must be at least one crossing point.

(Alternative Proof. Compare Figure 12. As we follow the bone $B_{-}(\mathbf{o})$ from one endpoint to the other, the kneading invariant $\mathcal{K}_{1}$ remains periodic, of order type $\mathbf{o}$. However, this kneading invariant cannot be the same at the two endpoints, since this would contradict Thurston's Theorem, and the only way for it to change is to pass a point, where both critical points belong to this same periodic orbit.)

It follows from simple plane topology, using the Jordan curve theorem, that the number of crossing points must be even, so there are at least two. Finally, there cannot be more than two crossing points, since each crossing point corresponds to a postcritically finite cubic map, which is uniquely determined by its kneading data $\mathbf{K}$. There are only two crossing points in the stunted sawtooth case, hence there are only two possible choices for $\mathbf{K}$, and the conclusion follows.

Definition. It will be convenient to divide each strictly $\boldsymbol{\sigma}$-bimodal bone $B_{ \pm}(\mathbf{o})$ into two halves by cutting at the primary intersection point. Each of these two will be called a half-bone.

Theorem 8.4. In either the cubic or the stunted sawtooth family, as we follow any left half-bone from its primary intersection endpoint to the edge $p_{2}=1$, the negative orbit complexity $\mathcal{N}$ increases monotonically, with an actual increase every time we cross a right bone. Again there is a one-to-one correspondence between crossing points in the two families which preserves the order along the half-bone, and which preserves kneading data.

The proof is completely analogous to the proof of 8.2. As we follow the half-bone, the kneading sequence $\mathcal{K}_{1}$ remains constant. Hence an orbit of negative type can appear or disappear only by passing through a parameter value for which the right folding point is periodic. Again there can be only one such parameter value for each periodic itinerary of negative type. Details will be left to the reader.

It follows that topological entropy increases monotonically as we traverse either left halfbone, starting at the primary intersection point. Again there is a completely analogous statement for right half-bones.

Definition. By the $n$-skeleton $S_{n}^{\text {saw }}$ for the stunted sawtooth family of some specified shape, either $\boldsymbol{\sigma}=(+-+)$ or $\boldsymbol{\sigma}=(-+-)$, we will mean the union of all bones $B_{ \pm}^{\text {saw }}(\mathbf{o}) \subset$ $P^{\text {saw }}$ of period $p \leq n$, together with the boundary $\partial P^{\text {saw }}$. Similarly, the $n$-skeleton $S_{n}^{\text {cub }}$ for the cubic family of the same shape is the union of all cubic bones $B_{ \pm}^{\text {cub }}(\mathbf{o})$ of period $p \leq n$, together with $\partial P^{\text {cub }}$. For elements of the analysis of the structure of these skeletons, see [RS], [RT], [MaT1], [MaT2] as well as [Mu].

It will be convenient to exclude the low period bones (those whose orbit types are not strictly bimodal), since they behave rather awkwardly. (Compare Figure 14.) Thus, in either family, we define the essential $n$-skeleton $E_{n}^{\text {saw }}$ or $E_{n}^{\text {cub }}$ to be the boundary of the 

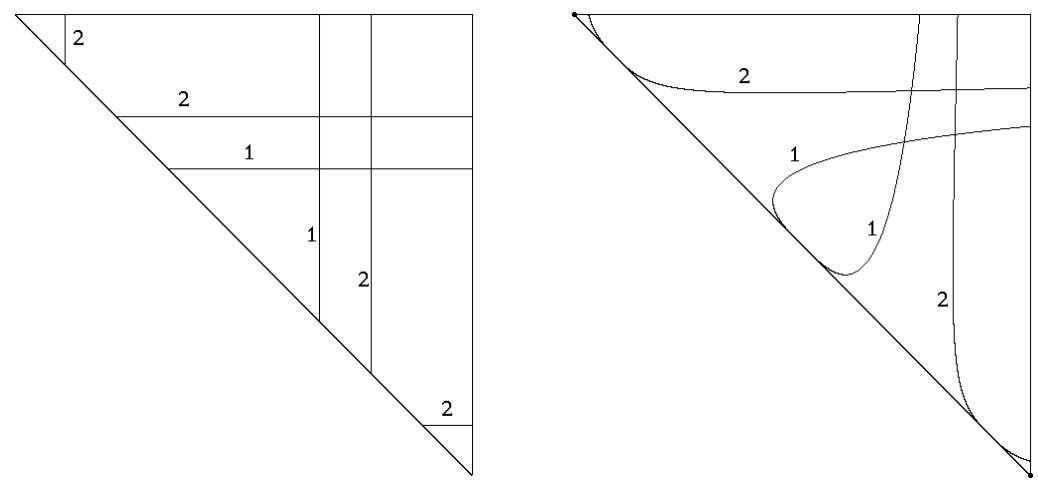

Figure 14. The bones of period $\leq 2$ for the shape $(-+-)$ are not strictly bimodal, and hence are quite atypical. In particular, their structure in the stunted sawtooth case (shown on the left) is qualitatively different from that in the cubic case (on the right). The period one bones for the $(+-+)$ case are even simpler, with no intersection point at all. In order to get a homeomorphism from one parameter triangle to the other preserving these low period bones, we would have to first remove the bottom edge of the triangle or collapse it to a point.

parameter triangle together with the union of all bones associated with strictly $\boldsymbol{\sigma}$-bimodal order types $\mathbf{o}$ of period $p \leq n$. In other words, we require that $2 \leq p \leq n$ in the $(+-+)$ case, and that $3 \leq p \leq n$ in the $(-+-)$ case. By a vertex of this essential skeleton, we mean either an endpoint of one of its bones or an intersection point of two of its bones.

As an example, Figure 13 shows the essential 4-skeletons for the stunted sawtooth and cubic families of shape $(-+-)$. In comparing the pictures for these two families, it is important that we consider only the essential skeletons, since low period bones do not behave in quite the same way.

Theorem 8.5 For either bimodal shape $\boldsymbol{\sigma}$, and for any $n>2$ there is a homeomorphism from the parameter triangle $P^{\text {saw }}$ to the parameter triangle $P^{\text {cub }}$ which maps the essential skeleton $E_{n}^{\text {saw }}$ homeomorphically onto the essential skeleton $E_{n}^{\text {cub }}$, carrying each bone of order type $\mathbf{o}$ to a bone of the same order type $\mathbf{o}$, carrying each vertex to a vertex with the same kneading data, and carring each edge of the parameter triangle to the corresponding edge.

The proof, making use of 8.2 and 8.4 and the fact that all intersections are transverse, is a straightforward exercise in plane topology. For example one can proceed inductively, starting with the identity map of $P^{2}$ and then adjusting it to behave correctly on one bone at a time. Details will be left to the reader. 


\section{$\S$ 9. From Connected Bones to Connected Isentropes.}

As usual. we fix one of the two possible shapes $\boldsymbol{\sigma}$ for bimodal maps. Working either in the cubic family or the stunted sawtooth family, we have the following.

Lemma 9.1. Let $\mathbf{p}$ and $\mathbf{p}^{\prime}$ be two points in the parameter triangle $P^{2}$ such that the associated maps have topological entropy $h\left(f_{\mathbf{p}}\right) \neq h\left(f_{\mathbf{p}^{\prime}}\right)$. Then any path from $\mathbf{p}$ to $\mathbf{p}^{\prime}$ in the parameter triangle $P^{\text {cub }}$ must cross infinitely many bones.

Proof of 9.1 According to Theorem 4.11 the difference $\left|\operatorname{Neg}\left(f_{\mathbf{p}}^{\circ k}\right)-\operatorname{Neg}\left(f_{\mathbf{p}^{\prime}}^{\circ k}\right)\right|$ must be unbounded as $k \rightarrow \infty$. As we deform $\mathbf{p}$ along some path in $P^{2}$, the number $\operatorname{Neg}\left(f_{\mathbf{p}}^{\circ k}\right)$, which measures the number of decreasing laps of $f_{\mathbf{p}}^{\circ k}$ whose graph crosses the diagonal, will remain constant except as we pass through a map which has a folding point with periodic orbit of period $q$ dividing $k$, with $k / q$ odd, in which case this number jumps by $\pm q$. (Compare 7.4.) Thus, $\operatorname{Neg}\left(f^{\circ k}\right)$ remains constant unless we pass through a bone of period dividing $k$. Further details are easily supplied.

Associated with the essential skeleton $E_{n}^{\text {cub }}$ or $E_{n}^{\text {saw }} \subset P^{2}$ is a topological cell structure on $P^{2}$. That is, we can partition $P^{2}$ into subsets, each of which is homeomorphic either to a point, an open interval, or a 2-dimensional open unit disk. Furthermore, these subsets fit together nicely so that the closure of each one is topologically a point, a closed interval, or a closed 2-disk. By definition, the open 2-cells in this cell structure are the connected components of the complement $P^{2} \backslash E_{n}$, the 0-cells are the vertices as described in Theorem 8.5, and the open 1-cells are the connected components of $E_{n} \backslash$ \{vertices\}. The resulting

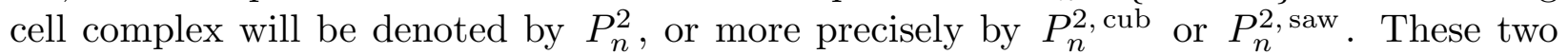

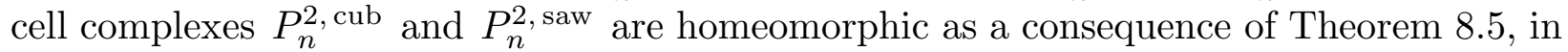
a homeomorphism $\eta_{n}$ which takes each vertex to a vertex of the same topological entropy and each edge to an edge with the same interval of entropies.

Lemma 9.2. For each $\epsilon>0$ there exists an integer $n$ such that if two points

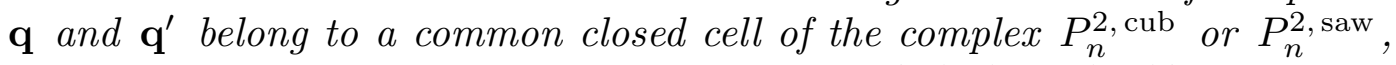
then the corresponding maps $f_{\mathbf{q}}$ and $f_{\mathbf{q}^{\prime}}$ satisfy $\left|h\left(f_{\mathbf{q}}\right)-h\left(f_{\mathbf{q}^{\prime}}\right)\right|<\epsilon$.

Proof in either family. Otherwise we could find $\epsilon>0$ so that for each $n$ there existed points $\mathbf{q}_{n}$ and $\mathbf{q}_{n}^{\prime}$ in a common cell of $P_{n}^{2}$ with $\left|h\left(f_{\mathbf{q}_{n}^{\prime}}\right)-h\left(f_{\mathbf{q}_{n}}\right)\right| \geq \epsilon$. After passing to infinite subsequences, we could assume that both sequences converge, say $\mathbf{q}_{k} \rightarrow \mathbf{q}$ and $\mathbf{q}_{k}^{\prime} \rightarrow \mathbf{q}^{\prime}$, and furthermore that all of the $\mathbf{q}_{k}$ and $\mathbf{q}_{k}^{\prime}$ belong to a common closed cell of $P_{n}$ whenever $k \geq n$. Thus the limit points $\mathbf{q}$ and $\mathbf{q}^{\prime}$ would belong to a common closed cell of $P_{n}^{2}$ for every $n$, but by continuity the associated entropies would differ by at least $\epsilon$. This is impossible by Lemma 9.1 .

Lemma 9.3. The topological entropy function $\mathbf{q} \mapsto h\left(f_{\mathbf{q}}\right)$, either for the cubic family or the stunted sawtooth family, restricted to any closed cell of the cell complex $P_{n}^{2}$, takes its maximum and minimum values on the boundary (and in fact on the set of boundary vertices). Hence the interval of entropy values which are realized by any cell in the cubic family is the same as the 
interval of entropy values for the corresponding cell in the stunted sawtooth family.

Proof. For the stunted sawtooth family, the first statement follows easily from the fact that entropy is a monotone function of either coordinate $p_{1}$ or $p_{2}$. To prove the analogous statement for the cubic family, suppose for example that for some point $\mathbf{p}_{0}$ of a closed cell $C$, the value $h\left(f_{\mathbf{p}_{0}}\right)$ were strictly larger than the maximum value $h_{\max }$ on the boundary of $C$. Let $2 \epsilon=h\left(f_{\mathbf{p}_{0}}\right)-h_{\max }$. According to Lemma 9.2 we can choose $m>n$ so that $h$

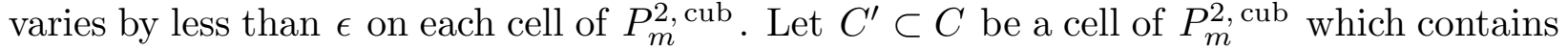
this point $\mathbf{p}_{0}$, and let $\mathbf{p}^{\prime}$ be any vertex of $C^{\prime}$. Then it follows that $h\left(f_{\mathbf{p}^{\prime}}\right)>h_{\max }$. Since the homeomorphism $\eta_{m}$ carries vertices to vertices with the same topological entropy, this

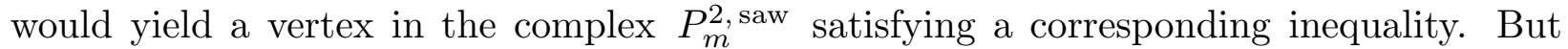
this is impossible, since we already proved Lemma 9.3 to be true for the stunted sawtooth family. The second statement follows immediately.

Main Theorem 9.4. Every isentrope $\left\{\mathbf{p} \in P_{ \pm}^{2, \text { cub }} ; h\left(f_{\mathbf{p}}\right)=h_{0}\right\}$ for either cubic family is connected.

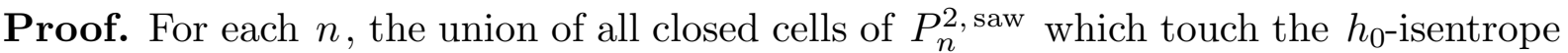
forms a compact set, which is connected by Theorem 6.1. The union of corresponding cells

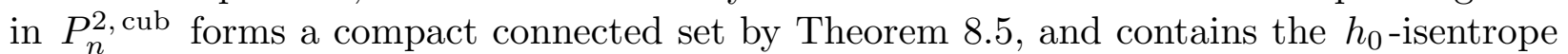
for the cubic family by Lemma 9.3. The intersection of these sets, as $n \rightarrow \infty$, will be precisely equal to the required isentrope by Lemma 9.2. Since an intersection of compact connected sets is compact and connected, the conclusion follows.

Recall that a compact subset of $\mathbb{R}^{n}$ is called cellular if it is the intersection of a nested sequence of closed topological $n$-cells, each contained in the interior of its predecessor. (Compare $[\mathrm{Br}]$.

Corollary 9.5. Each isentrope $\left\{h=h_{0}\right\}$ for either cubic family is a cellular set. Further, if $0<h_{0}<\log (3)$ then this isentrope separates the parameter triangle into two connected subsets.

Proof. Since we are in dimension two, it is only nessary to check that a set is compact and connected, with connected complement in $\mathbb{R}^{2}$, in order to prove that it is cellular. But it follows from 9.4 that no isentrope can separate the plane. For otherwise, if $\mathbf{p}_{0}$ belonged to the bounded component of the complement of some isentrope $\left\{h=h_{0}\right\} \subset P^{2}$ within $\mathbb{R}^{2}$, then choosing a point on the boundary of the parameter triangle with the same topological entropy as $p_{0}$, we would see that the isentrope $\left\{h=h\left(\mathbf{p}_{0}\right)\right\}$ could not be connected, contradicting 9.4. Since we know by 8.2 that each isentrope intersects the two top edges of the parameter triangle in connected sets, it follows easily that the complement $P^{2} \backslash\left\{h=h_{0}\right\}$ has at most two connected components. 


\section{Appendix A : Characterization of a Polynomial by its Critical Values.}

A letter from Adrien Douady (slightly edited).

Tuesday, 13 July 1993

Dear Milnor,

Putting order in my papers, I found some notes I had written with Pierrette Sentenac after a talk by Arnold in ENS, where he asked for a proof in $\mathbb{R}$. Let:

$\mathcal{P}_{n}=\{$ monic centered real polynomials of degree $n\}=\left\{x^{n}+c_{n-2} x^{n-2}+\cdots+c_{0}\right\}$,

$\Omega=\left\{f \in \mathcal{P}_{n} \mid f\right.$ has distinct real roots $\left.a_{1}, \ldots, a_{n}\right\}$.

Then

$f \mapsto\left(a_{1}, \ldots, a_{n}\right)$ is a diffeo of $\Omega$ onto $\left\{\left(a_{1}, \ldots, a_{n}\right) \mid a_{1}<\cdots<a_{n}, \sum a_{i}=0\right\}$,

or equivalently

$f \mapsto\left(\ell_{1}, \ldots, \ell_{n-1}\right)$, where $\ell_{i}=a_{i+1}-a_{i}$, is a diffeo of $\Omega$ onto $\left(\mathbb{R}_{+}\right)^{n-1}$.

Let $\Phi(f)=\left(s_{1}, \ldots, s_{n-1}\right)$ where

$$
s_{i}=\int_{a_{i}}^{a_{i+1}}|f(x)| d x=(-1)^{n-i} \int_{a_{i}}^{a_{i+1}} f(x) d x .
$$

Proposition. $\Phi: f \mapsto\left(s_{1}, \ldots, s_{n-1}\right)$ is a diffeo of $\Omega$ onto $\left(\mathbb{R}_{+}\right)^{n-1}$.

Proof. a) $\Phi$ is a local diffeo. In fact, for $g$ of degree $n-2$, we have

$$
(-1)^{n-i} \frac{d \Phi_{i}(f+\epsilon g)}{d \epsilon}=\int_{a_{i}}^{a_{i+1}} g(x) d x+\frac{d a_{i+1}}{d \epsilon} f\left(a_{i+1}\right)-\frac{d a_{i}}{d \epsilon} f\left(a_{i}\right)
$$

at $\epsilon=0$, where the last two summands vanish since the $a_{i}$ are zeros of $f$. Thus $d \Phi /\left.d \epsilon\right|_{\epsilon=0}$ could vanish only if $g$ had zero average on each of the $(n-1)$ intervals $\left[a_{i}, a_{i+1}\right]$, and hence had at least $n-1$ roots; which is impossible for $g \neq 0$ of degree $n-2$.

b) $\Phi$ is proper. In fact $s_{i} \rightarrow \infty$ as the length $\ell_{i}=a_{i+1}-a_{i}$ tends to $\infty$, and $s_{i} \rightarrow 0$ as $\ell_{i}$ tends to 0 with $\sum \ell_{j}$ bounded. (Use the fact that $f(x)=\prod\left(x-a_{i}\right)$.)

Corollary. Let $\Omega^{\prime} \subset \mathcal{P}_{n+1}$ be the set of monic centered polynomials $F$ of degree $n+1$ with $n$ distinct real critical points. Taking $(n+1) f$ to be the derivative of $F$, it follows that the map $F \mapsto\left(v_{1}, \ldots, v_{n}\right)$ from $\Omega^{\prime}$ to $\mathbb{R}^{n}$, where $v_{i}$ are the critical values (in the order of the critical points) is a diffeo of $\Omega^{\prime}$ onto the set of $\left(v_{1}, \ldots, v_{n}\right)$ with $\operatorname{sgn}\left(v_{i}-v_{i-1}\right)=(-1)^{n-i}$.

Best wishes,

Adrien 
Addendum. This argument can easily be extended to the case where the roots $\left\{a_{j}\right\}$ of $f$ (alias the critical points of $F$ ) need not be distinct, but must only satisfy $a_{1} \leq a_{2} \leq \cdots \leq a_{n}$ with $\sum a_{i}=0$. Let $\hat{a}_{1}<\cdots<\hat{a}_{r}$ be the distinct roots of $f$, with multiplicities $m_{i} \geq 1$ so that

$$
f(x)=\left(x-\hat{a}_{1}\right)^{m_{1}} \cdots\left(x-\hat{a}_{r}\right)^{m_{r}},
$$

with

$$
m_{1}+\cdots+m_{r}=n \quad \text { and } \quad m_{1} \hat{a}_{1}+\cdots+m_{r} \hat{a}_{r}=0 .
$$

To deform $f$ within polynomials of this same form, choose direction parameters $w_{i} \in \mathbb{R}$ with $m_{1} w_{1}+\cdots+m_{r} w_{r}=0$ and set

$$
f_{t}(x)=\left(x-\hat{a}_{1}(t)\right)^{m_{1}} \ldots\left(x-a_{r}(t)\right)^{m_{r}} \quad \text { where } \quad \hat{a}_{i}(t)=\hat{a}_{i}+t w_{i} .
$$

As before, let

$$
s_{i}(t)=\int_{\hat{a}_{i}(t)}^{\hat{a}_{i+1}(t)}\left|f_{t}(x)\right| d x= \pm \int_{\hat{a}_{i}(t)}^{\hat{a}_{i+1}(t)} f_{t}(x) d x .
$$

A brief computation shows that the derivative $d s_{i} / d t$ at $t=0$ can be expressed as the integral from $\hat{a}_{i}$ to $\hat{a}_{i+1}$ of the product of a fixed polynomial

$$
\mp\left(x-\hat{a}_{1}\right)^{m_{1}-1} \cdots\left(x-\hat{a}_{r}\right)^{m_{r}-1}
$$

which has no zeros within this interval, and a polynomial

$$
g(x)=\sum_{i} m_{i} w_{i} \prod_{\{j ; j \neq i\}}\left(x-\hat{a}_{j}\right)
$$

of degree $\leq r-2$. This polynomial $g(x)$ is non-zero unless all of the $w_{i}$ are zero, since

$$
g\left(\hat{a}_{i}\right)=m_{i} w_{i} \prod_{\{j ; j \neq i\}}\left(\hat{a}_{i}-\hat{a}_{j}\right)
$$

which is non-zero unless $w_{i}=0$. Hence $g$ has at most $r-2$ zeros. Arguing as above, it follows that the linear first derivative map

$$
\left.\left(w_{1}, \ldots, w_{r}\right) \mapsto\left(d s_{1} / d t, \ldots, d s_{r-1} / d t\right)\right|_{t=0}
$$

is bijective, and it follows as before that $F$ is uniquely determined by its critical value vector $\left(v_{1}, \ldots, v_{n}\right)$, where now the case $v_{i}=v_{i+1}$ is allowed.

\section{Appendix B: Tight Symbol Sequences and Thurston's Theorem.}

We first make a rather formal definition, to be justified by the properties which follow. Let $\mathcal{I}(x)=\left(A_{0}, A_{1}, A_{2}, \ldots\right) \in \mathfrak{A}^{\mathbb{N}}$ be the itinerary of a point under some $m$-modal map of shape $\boldsymbol{\sigma}$ with kneading data $\mathbf{K}=\left(\mathcal{K}_{1}, \ldots, \mathcal{K}_{m}\right)$.

Definition. We will say that this symbol sequence $\mathcal{I}(x)=\left(A_{0}, A_{1}, \ldots\right)$ is flabby (flasque) if some terminal segment

$$
\operatorname{shift}^{\circ k} \mathcal{I}(x)=\left(A_{k}, A_{k+1}, \ldots\right)
$$

has either the form $\left(I_{j}, \mathcal{K}_{j}\right)$ or the form $\left(I_{j-1}, \mathcal{K}_{j}\right)$, for any $1 \leq j \leq m$. In other words, some point of the associated orbit which is not a folding point must have the same itinerary 
as an immediately adjacent folding point. This symbol sequence will be called tight if it is not flabby. Similarly, we will say that the kneading data $\mathbf{K}$ is tight if each of the sequences $\mathcal{K}_{1}, \ldots, \mathcal{K}_{m}$ is tight.

As a first example, consider the family of stunted sawtooth maps with fixed shape $\boldsymbol{\sigma}$ and fixed slope $s>m+1$.

Lemma B.1. Let $S_{\mathbf{p}}: J \rightarrow J$ be a stunted sawtooth map in this family with kneading data $\mathbf{K}$, and let $\mathcal{I}^{0}$ be a symbol sequence in $\mathfrak{A}^{\mathbb{N}}$. There exists one and only one $x \in J$ with itinerary $\mathcal{I}^{0}$ if and only if this symbol sequence is tight and $\mathbf{K}$-admissible.

Similarly, for any kneading data $\mathbf{K}$, there exists one and only one map $S_{\mathbf{p}}$ in the family with this kneading data if and only if $\mathbf{K}$ is tight and $\boldsymbol{\sigma}$ admissible.

(Existence was shown in $\S 5$, so only the uniqueness part is new and requires tightness.) Proofs will be given at the end of this appendix. We can also give a more geometric criterion for tightness in the stunted sawtooth case.

Lemma B.2. The itinerary of $x$ under $S_{\mathbf{p}}$ is tight if and only if the orbit of $x$ never hits any plateau except at its central folding point. Similarly the kneading data for $S_{\mathbf{p}}$ is tight if and only if the orbit of each folding point satisfies this condition.

Recall from $\S 5$ that any admissible kneading data is represented by a canonical stunted sawtooth map $S_{\mathbf{p}}$, which has the property that the orbit of a folding point cannot hit the interior of a plateau except at a folding point. Thus, if we use this canonical map, the only extra requirement for tightness of $\mathbf{K}$ is that the folding point orbits cannot even hit the endpoints of the plateaus.

The case of polynomial maps is much more difficult, and not well understood. However, for the postcritically finite case (where every critical orbit is periodic or eventually periodic) we have the following result ${ }^{10}$ which is an adaptation of a basic theorem of Thurston in a form due to Poirier.

Theorem B.3. Suppose that the $m$-modal kneading data $\mathbf{K}$ is $\boldsymbol{\sigma}$-admissible, with $\mathcal{K}_{i} \neq \mathcal{K}_{i+1}$ for all $i$. There exists a postcritically finite polynomial map of degree $m+1$ and shape $\boldsymbol{\sigma}$ with kneading data $\mathbf{K}$ if and only if each $\mathcal{K}_{i}$ is periodic or eventually periodic, and also tight. This polynomial is always unique when it exists, up to a positive affine change of coordinates, or as a boundary anchored map of the interval.

To illustrate this result, here are four examples of non-tight data. The notation $\bar{A}$ will stand for an infinite sequence $(A, A, A, \ldots)$ of identical copies of the symbol $A \in \mathfrak{A}$.

\footnotetext{
${ }^{10}$ Erratum. This result was stated incorrectly, without the hypothesis of tightness, in our preliminary manuscript [DGMT, §5]. A counter-example to the version stated there is provided in Example 3 below. (Figure 15-c.)
} 

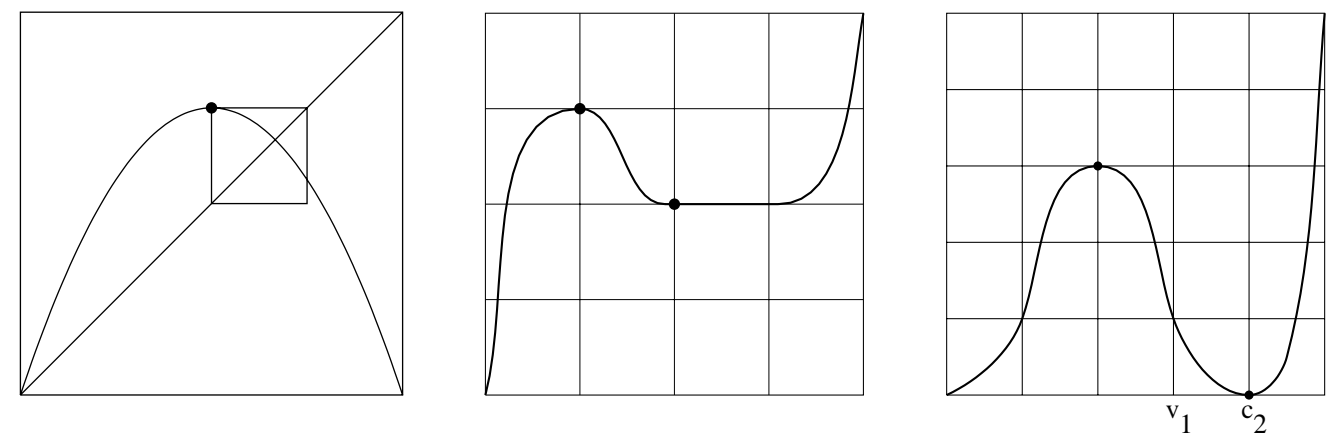

Figure 15. Three maps with eventually periodic but non-tight kneading data. (See Examples 1, 2, 3.) The folding points are marked.

Example 1. If $2<4 v<1+\sqrt{5}$, the unimodal map $Q_{v}(x)=4 v x(1-x)$ has periodic kneading sequence $\mathcal{K}_{1}=\bar{I}_{1}=\left(I_{1}, \mathcal{K}_{1}\right)$. This kneading sequence is not tight. It is realised by an entire one-parameter family of distinct quadratic maps (Figure 15-a), but not by any postcritically finite quadratic map.

Example 2. The $(+-+)$-bimodal kneading data $\mathcal{K}_{1}=\left(I_{2}, \bar{C}_{2}\right), \mathcal{K}_{2}=\bar{C}_{2}$ is admissible, but cannot be realised by any piecewise strictly monotone map. (Figure 15-b. Compare the discussion of 8.1.)

Example 3. The $(+-+)$-bimodal data $\mathcal{K}_{1}=\left(I_{1}, \bar{I}_{0}\right), \mathcal{K}_{2}=\bar{I}_{0}$ can be realised by a postcritically finite 4 -th degree polynomial, but not by any cubic polynomial. (Figure 15-c.)

Example 4. (See [MaT1, p. 179].) More generally, whenever the kneading data for $f$ satisfies $\mathcal{K}_{1}=\left(I_{1}, \mathcal{K}_{2}\right)$, it follows that all points in the open interval $\left(v_{1}, c_{2}\right) \subset I_{1}$ have the same itinerary. If $\mathcal{K}_{2}$ is not eventually periodic, then $\left(v_{1}, c_{2}\right)$ is a wandering interval, hence $f$ cannot be real analytic. ${ }^{11}$ There are uncountably many distinct choices for $\mathcal{K}_{2}$. In fact, using the stunted sawtooth model with folding points \pm 1 , we can take $v_{1}=1-\epsilon$ and let $v_{2}$ range from $-\alpha$ to $v_{1}$. The corresponding topological entropy ranges between $\log 2$ and zero. (For example, the right picture in Figure 15 illustrates the $\log 2$ case.) Thus we can certainly choose infinitely many $\mathcal{K}_{2}$ which are not eventually periodic.

For the application, we need a special case where the hypothesis of tightness is automatically satisfied:

Corollary B.4. Let $\mathbf{K}$ be admissible kneading data such that every kneading sequence $\mathcal{K}_{j}$ either

1) contains the symbol $C_{j}$, so that the folding point itinerary $\left(C_{j}, \mathcal{K}_{j}\right)$ is periodic, or

2) satisfies the condition that $\mathcal{K}_{j}$ equals either $\mathcal{I}_{\min }$ or $\mathcal{I}_{\max }$ according as the sign $\sigma_{j}$ is +1 or -1 .

\footnotetext{
${ }^{11}$ Compare [L1], [BL], [MvS1], [MMS]. It would be interesting to know whether any such kneading data can be realized by a map which is $C^{\infty}$ and piecewise strictly monotone. Examples of $C^{\infty}$ maps with wandering intervals have been given in in [Ha] and [SI].
} 
Then this data is tight, and hence is realized by one and only one postcritically finite polynomial in the parametrized family of $\S 3$, and by one and only one stunted sawtooth map in the family of $\S 5$.

(However, compare Figure 16.) Here the various periodic critical orbits need not be disjoint. It follows that there is a canonical one-to-one correspondence between intersection of bones in the cubic family and in the corresponding stunted sawtooth family, and also between endpoints of bones in these two families. This would yield an alternate proof of 8.5 .
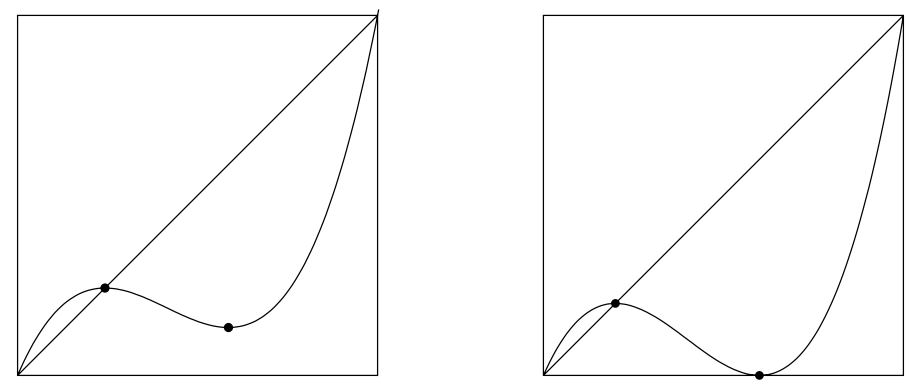

Figure 16. The periodic kneading data $\mathcal{K}_{1}=\bar{C}_{1}, \mathcal{K}_{2}=\bar{I}_{0}$ is tight, yet is represented by an entire one-parameter family of cubic maps. However, only one of these is postcritically finite.

The proofs follow.

Proof of B.1 and B.2. First note that the two statements B.1 and B.2 are equivalent to each other: The itinerary of any non folding point on the $j$-th plateau is either $\left(I_{j-1}, \mathcal{K}_{j}\right)$ or $\left(I_{j}, \mathcal{K}_{j}\right)$. If the orbit of $x$ hits each plateau at most at its center point, then as in $\S 5$ one can compute $x$ from its itinerary, and it is not difficult to see that this itinerary cannot contain any $\left(I_{j-1}, \mathcal{K}_{j}\right)$ or $\left(I_{j}, \mathcal{K}_{j}\right)$.

If the orbit of $x$ hits some plateau off-center, then clearly we can move $x$ slightly without changing its itinerary. Similarly, if the $i$-th folding point orbit hits the interior of some plateau at a non folding point, then varying the $i$-th folding value but keeping all other folding values fixed we obtain a one-parameter family of stunted sawtooth maps with the same kneading data. If, for one or more values of $i$, the $k(i)$-th forward image of the folding value $v_{i}$ lies at an endpoint of the $j(i)$-th plateau, then the argument is slightly more delicate: Vary all such folding values at unit speed in such a way that this $k(i)$-th forward image moves with speed $s^{k(i)} \geq 1$ towards the interior of the corresponding plateau. Of course the $j(i)$-th folding value may itself be varying at unit speed, but in this case the endpoints of the plateau will then move at speed $1 / s<1$, so that the image will still move into the interior of the plateau, and the kneading data will not change. Thus $S_{\mathbf{p}}$ is not tight.

Conversely, if the orbit of $x$ hits every plateau at most at its center point, then it follows from the remarks above that $x$ is tight, and the corresponding statement for kneading data follows easily. 
Proof of B.3. We will make use of a very special form of Hubbard tree, as described by Douady and Hubbard [DH1], and in a more precise form by Poirier [Po1, Theorem A]. Since we are interested only in real maps with non-degenerate critical points, our Hubbard tree $T$ can be identified simply with an interval $[1, n]$ of real numbers, with the integer points $1,2, \ldots, n$ as vertices. Here $m$ of these vertices are to be designated as critical points (hence $n \geq m$ ). As final element of structure, there is to be a mapping $F$ from this finite set $\{1,2, \ldots, n\}$ to itself which satisfies three conditions:

(i) $F$ must take consecutive vertices to distinct points.

(ii) Extending linearly over the intermediate intervals $[i, i+1]$, we require that two consecutive intervals should map with opposite orientations (so that their images overlap) if and only their common vertex is a critical point.

The third condition can be stated in several equivalent forms (compare [Po1, II.3.11]), but the following will be convenient for our purposes.

(iii) Poirier Expansion Condition. For any interval $[i, i+1] \subset T$ there must be an integer $k \geq 0$ so that the $k$-th forward image of this interval contains a critical point.

Assertion: These conditions are satisfied if and only if there is a polynomial map $f$ of degree $m+1$, necessarily unique up to positive affine conjugacy, and an order preserving embedding of the vertices $\{1,2, \ldots, n\}$ of $T$ into the real line which sends the designated critical points in $T$ to the critical points of $f$, and which conjugates $F$ (on this set of vertices) to $f$.

Poirier's proof (of a more general theorem which implies this Assertion) is based on Thurston's characterization of postcritically finite rational maps (see [DH2]), and relies on earlier work by Douady, Hubbard, Bielefeld, Y. Fisher, and others (see [Po2] and references therein).

To apply this result to the proof of B.3, we start with the finite subset $\Sigma \subset \mathfrak{A}^{\mathbb{N}}$ consisting of the critical orbits $\left(C_{j}, \mathcal{K}_{j}\right)$ together with all of their terminal segments. Number the elements of $\Sigma$ in increasing order from 1 to $n$, and let the shift map on $\Sigma$ correspond to the mapping $F$ on $\{1, \ldots, n\}$. Then Condition (i) above follows from tightness, together with the hypothesis that $\mathcal{K}_{i} \neq \mathcal{K}_{i+1}$, while Condition (ii) follows from admissibility, and Condition (iii) follows trivially from the definition of kneading sequences as itineraries. Thus the Assertion above yields a polynomial map $f: \mathbb{R} \rightarrow \mathbb{R}$. Let $I \subset \mathbb{R}$ be the set of points with bounded orbit. Since $f$ is postcritically finite, all critical orbits are bounded. It follows easily that $I$ is a closed interval, and that $\left.f\right|_{I}$ is boundary anchored. There cannot be any smaller closed interval $J \subset I$ which contains all critical points and is $f$-invariant and boundary anchored. For then a component of $\overline{I \backslash J}$ would contain an attracting point (of period $\leq 2$ ), which is impossible in the postcritically finite case.

Closely related is an alternative characterization of tightness, in the eventually periodic case.

Corollary B.5. Let $\mathbf{K}$ be admissible eventually periodic kneading data with $\mathcal{K}_{i} \neq \mathcal{K}_{i+1}$ for all $i$. Then $\mathbf{K}$ is tight if and only if the collection of all 
terminal segments of the folding point itineraries $\left(C_{i}, \mathcal{K}_{i}\right)$ has the property that consecutive elements (in the ordering of §2) map to distinct points under the shift map.

Proof. This follows immediately from the discussion above.

Proof of B.4. Although it is not difficult to prove B.4 directly from the definition of tightness, the argument would be completely formal. It is more intuitive to make use of B.5. In other words, we look at the associated Hubbard tree, whose set of vertices can be identified with the union of the critical orbits for a representative map, and check directly that consecutive vertices map to distinct points. For the union of the periodic critical orbits, there is nothing to prove since distinct points clearly map to distinct points. Any two critical points which map to the right endpoint, corresponding to $\mathcal{I}_{\max }$, have the same parity, and hence are separated by another critical point. We must also check that the rightmost critical point and the $\mathcal{I}_{\text {max }}$ point have distinct images, $\mathcal{K}_{m} \neq \operatorname{shift}\left(\mathcal{I}_{\text {max }}\right)$. But if $\sigma_{m}=+1$ then $\operatorname{shift}\left(\mathcal{I}_{\max }\right)=\mathcal{I}_{\max } \neq \mathcal{K}_{m}$, while if $\sigma_{m}=-1$ then $\operatorname{shift}\left(\mathcal{I}_{\max }\right)=\mathcal{I}_{\min } \neq$ $\mathcal{K}_{m}$, using the hypothesis of B.4 in both cases. The discussion of critical points mapping to $\mathcal{I}_{\min }$ is completely analogous.

\section{Appendix C: Monotonicity vs Antimonotonicity.}

As we mentioned in $\S 8$, one nice monotonicity property in parametrized families would be to have a line through each point in the parameter space such that the dynamical complexity increases monotonically along the line. This augmentation of complexity could be precisely associated to the fact that no periodic orbit disappears as we follow the line: in one dimension this implies (but is not required for) monotonicity of the topological entropy. For the quadratic family, it is true that no periodic orbit disappears. (If one does not like to follow deformation of orbits from their birth or to their destruction, one can think of the equivalent statement that the number of orbits of each order type does not decrease). Now think of the quadratic maps as singular maps of $\mathbb{R}^{2}$, writing $\tilde{Q}_{v, 0}(x, y)=(4 v x(1-x)+y, 0)$. Such maps are limits of diffeomorphisms of the plane, the Hénon maps, obtained for each $b \neq 0$ by writing $\tilde{Q}_{v, b}(x, y)=(4 v x(1-x)+y, b y)$. Numerical computations have suggested for a long time that periodic orbits both appear and disappear along typical one-parameter families extracted from the two-parameter Hénon family. The deep reason for these observations was finally given by the following result (Compare $[\mathrm{KKY}]$ ).

Antimonotonicity Theorem. In any neighborhood of a nondegenerate, homoclinic-tangency parameter value of a one-parameter $C^{3}$-smooth family of dissipative diffeomorphisms of the plane, there must be both infinitely many orbit-creation and infinitely many orbit-annihilation parameter values.

It has been conjectured ([DG], [DGYKK], see also $[\mathrm{DGK}])$ that a similar statement should work for $m$-modal maps as soon as $m>1$. More precisely, calling antimonotone a parameter value approached both by infinitely many orbit-creation and infinitely many orbit-annihilation parameter values, the following can be extracted from [DGYKK]: 
Antimonotonicity Conjecture. A smooth one-dimensional map depending on one parameter has antimonotone parameter values whenever two critical points have disjoint orbits and are contained in the interior of a chaotic attractor.

This conjecture would not overtly contradict 9.4. If we follow a (not necessarily smooth) path within some isentrope, note that any significant amount of periodic orbit creation must be offset by a roughly equivalent amount of orbit annihilation, so that topological entropy will remain constant. Even if we could find a smooth curve which is transverse to the family of isentropes, so that topological entropy increases monotonically along it, it seems possible that orbit annihilations could be dense, but outweighed by even more orbit creations. Similarly, the Antimonicity Theorem above does not contradict the possibility that isentropes for the family of real Hénon maps may be connected, although we have no reason to conjecture this.

The analogue of the Antimonoticity Conjecture for the stunted sawtooth families is certainly false, since by 5.8 , it is very easy to find smooth curves along which there are only orbit creations. Thus, if the conjecture is true for the cubic family, then any complexity preserving correspondence between the stunteed sawtooth and cubic parameter triangles must be very wild indeed.

\section{References.}

[AKM] R.L. Adler, A.G. Konheim, and M.H. McAndrews, "Topological entropy", Trans. Amer. Math. Soc. 114 (1965) 309-319.

[ALM] L. Alsedà, J. Llibre, and M. Misiurewicz, Combinatorial Dynamics and Entropy in Dimension One, (World Scientific , Singapore, 1993).

[BCMM] C. Bernhardt, E. Coven, M. Misiurewicz, and I. Mulvey, "Comparing periodic orbits of maps of the interval", Trans. Amer. Math. Soc. 333 (1992) 701-707.

[Be] A.F. Beardon, A Primer on Riemann Surfaces, London Mathematical Society Lecture Notes Series 78 (Cambridge University Press, Cambridge, 1984).

$[\mathrm{BK}]$ L. Block and J. Keesling, "Computing topological entropy of maps of the interval with three monotone pieces", J. Stat. Phys. 66 (1992) 755-774.

[BL] A. M. Blokh and M. Lyubich, " Non-existence of wandering intervals and structure of topological attractors of one-dimensional dynamical systems II, The smooth case", Erg. Th. and Dynam. Sys. 9 (1989) 751-758.

[BMT] K. Brucks, M. Misiurewicz and C. Tresser, "Monotonicity properties of the family of trapezoidal maps", Commun. Math. Phys. 137 (1991) 1-12.

[Bo] R. Bowen, On Axiom A Diffeomorphisms, Proc. Reg. Conf. Math. 35, (1978).

[Br] M. Brown, "A proof of the generalized Schoenflies theorem", Bull. Amer. Math. Soc. 66 (1960) 74-76. (See also: M. Brown, "The monotone union of open $n$-cells is an open n-cell", Proc. Amer. Math. Soc. 12 (1961) 812-814.) 
[BR] V. Baladi and D. Ruelle, "An extension of the theorem of Milnor and Thurston on the zeta functions of interval maps", Ergodic Theory and Dynam. Sys. 14 (1994) 621-632.

[BST] N. J. Balmforth, E. A. Spiegel and C. Tresser, "The topological entropy of onedimensional maps: approximation and bounds", Phys. Rev. Lett. 80 (1994) 80-83.

[D] A. Douady, "Topological entropy of unimodal maps: Monotonicity for quadratic polynomials", pp. 65-87 of Real and Complex Dynamical Systems, (B. Branner and P. Hjorth Eds.) (Kluwer, Dordrecht, 1995).

[DG] S. P. Dawson and C. Grebogi, "Cubic maps as models of two-dimensional antimonotonicity", Chaos, Solitons \& Fractals 1 (1991), 137-144.

[DGK] S. P. Dawson, C. Grebogi and H. Koçak, "A geometric mechanism for antimonotonicity in scalar maps with two critical points", Phys. Rev. E 48 (1993) 1676-1682.

[DGKKY] S. P. Dawson, C. Grebogi, I. Kan, H. Koçak and J. A. Yorke, "Antimonotonicity: inevitable reversals of period doubling cascades", Phys. Lett.A 162 (1992) 249-254.

[DGMT] S. P. Dawson, R. Galeeva, J. Milnor and C. Tresser, "A monotonicity conjecture for real cubic maps", pp. 165-183 of Real and Complex Dynamical Systems, (B. Branner and P. Hjorth Eds.) (Kluwer, Dordrecht, 1995).

[DH1] A. Douady and J. Hubbard, "A proof of Thurston's topological characterization of rational maps", Acta Math. 171 (1993) 263-297.

[DH2] A. Douady and J. H. Hubbard, "Etude dynamique des polynômes quadratiques complexes", I (1984) \& II (1985), Publ. Mat. d'Orsay.

[E] A. Epstein, "Algebraic dynamics: contraction, finiteness, and transversality principles", manuscript in preparation. (See also "Towers of Finite Type Complex Analytic Maps", Thesis, CUNY, 1993.)

[F] P. Fatou, "Sur les équations fonctionnelles, II", Bull. Soc. Math. France 48 (1920) 33-94.

[FK] H.M. Farkas and I. Kra, Riemann Surfaces, Graduate Texts in Mathematics $\mathbf{7 1}$ (Springer Verlag, New York, Second Edition, 1992).

[FT] B. Friedman and C. Tresser, "Comb structure in hairy boundaries: some transition problems for circle maps", Phys. Lett. 117A (1986) 15-22.

[GS] J. Graczyk and G. Świątek, "Generic hyperbolicity in the logistic family", Annals of Math. 146 (1997) 1-52.

[Gu] J. Guckenheimer, in Dynamical Systems, C.I.M.E. Lectures (J. Guckenheimer, J. Moser and S. Newhouse), Progress in Mathematics 8, (Birkhauser, New York, 1980).

[Ha] G. R. Hall, "A $C^{\infty}$ Denjoy counterexample" Ergodic Theory and Dynamical Systems 1 (1981) 261-272.

[He] C. Heckman, "Monotonicity and the Construction of Quasiconformal Conjugacies in the Real Cubic Family", Thesis, Stony Brook, 1996.

[HKC] L.P. Hurd, J. Kari, and K. Culik, "The topological entropy of cellular automata is 
uncomputable", Erg. Th. and Dynam. Sys. 12 (1992) 255-265.

[K] V. Kaloshin, "Generic Diffeomorphisms with Superexponential Growth of Number of Periodic Orbits", Stony Brook I.M.S. Preprint 1999\#2.

[Ka] A. Katok, "Lyapunov exponents, entropy and periodic orbits of diffeomorphisms", Pub. Math. IHES 51 (1980) 137-173.

[KKY] I. Kan, H. Koçak and J. A. Yorke, "Antimonotonicity: Concurrent creation and annihilation of periodic orbits", Annals of Math. 136 (1992) 219-252.

[L1] M. Lyubich, "Non-existence of wandering intervals and structure of topological attractors of one-dimensional dynamical systems I, The case of negative Schwarzian derivative", Erg. Th. and Dynam. Sys. 9 (1989) 737-750.

[L2] M. Lyubich, "Dynamics of quadratic polynomials, I and II", Acta Math. 178 (1997) 185-247, 247-297.

[M1] J. Milnor, "Remarks on iterated cubic maps", Experimental Math. 1 (1992) 5-24.

[M2] J. Milnor, "Hyperbolic components in Spaces of Polynomial Maps (with an appendix by A. Poirier)", Stony Brook I.M.S. Preprint 1992\#3.

[M3] J. Milnor, "On cubic polynomials with periodic critical point", in preparation.

[MaT1] R. S. MacKay and C. Tresser, "Boundary of topological chaos for bimodal maps of the interval", J. London Math. Soc. 37 (1988), 164-181.

[MaT2] R. S. MacKay and C. Tresser, "Some flesh on the skeleton: the bifurcation structure of bimodal maps", Physica 27D (1987) 412-422.

[Mc1] C. McMullen, "Automorphisms of rational maps", pp. 31-60 of Holomorphic Functions and Moduli I, ed. Drasin, Earle, Gehring, Kra \& Marden; MSRI Publ. 10, (Springer, New York, 1988).

[Mc2] C. McMullen, Complex Dynamics and Renormalization, Ann. Math. Studies 135, (Princeton University Press, Princeton, 1994).

[Mis1] M. Misiurewicz, "On non-continuity of topological entropy", Bull. Ac Pol. Sci., Ser. Sci. Math. Astr. Phys. 19 (1971) 319-320.

[Mis2] M. Misiurewicz, "Horseshoes for mappings of an interval", Bull. Ac Pol. Sci., Ser. Sci. Math. Astr. Phys. 27 (1979) 167-169.

[Mis3] M. Misiurewicz, "Jumps of entropy in one dimension", Fund. Math. 132 (1989) $215-226$.

[Mis4] M. Misiurewicz, "Continuity of entropy revisited", Dynamical systems and applications, World Sci. Ser. Appl. Anal. 4 (1995) 495-503.

[MMS] M. Martens, W. de Mello, and S. van Strien, "Julia-Fatou-Sullivan theory for real one-dimensional dynamics", Acta Math. 168 (1992) 273-318.

[MN] M. Misiurewicz and Z. Nitecki, 'Combinatorial Patterns for Maps of the Interval, Memoirs of the A.M.S. 456 (1991). 
[MSS] N. Metropolis, M. L. Stein, and P. R. Stein "On finite limit sets for transformations on the unit interval", J. Comb. Theory 15 (1973) 25-44.

[MSz] M. Misiurewicz and W. Szlenk, "Entropy of piecewise monotone mappings", Studia Math. 67 (1980) 45-63 (Short version: Astérisque 50 (1977) 299-310).

[MTh] J. Milnor and W. Thurston, "On iterated maps of the interval", in Springer Lecture Notes 1342 (1988), 465-563.

[Mu] P. Mumbrú, Estructura Periòdica i Entropia Topològica de les Aplicacions Bimodals Thesis, Universitat Autònoma de Barcelona, 1987.

[MvS1] W. de Mello and S. van Strien, "A structure theorem in one-dimensional dynamics", Annals of Math. 129 (1989) 519-546.

[MvS2] W. de Melo and S. Van Strien, One Dimensional Dynamics, (Springer Verlag, Berlin, 1993).

[My] P. J. Myrberg, "Iteration der reellen Polynome zweiten Graden", Ann. Acad. Sci. Fennic. 256A (1958) 1-10, 268A (1959) 1-13, and 336A (1963) 1-18.

[N] S. Newhouse, "Continuity properties of entropy", Annals of Math. 129 (1989) 215-235 and 131 (1990) 409-410.

[NN], K. Nishizawa and A. Nojiri, "Center curves in the moduli space of the real cubic maps", Proc. Japan Acad. Ser. A Math. Sci. 69 (1993) 179-184. See also: "Algebraic geometry of center curves in the moduli space of the cubic maps", Proc. Japan Acad. Ser. A Math. Sci. 70 (1994) 99-103.

[Po1] A. Poirier, "On post critically finite polynomials, Part II, Hubbard Trees", Stony Brook I.M.S. Preprint 1993\#7. (Thesis, Stony Brook 1993.)

[Po2] A. Poirier, "Realizing reduced schemata", Appendix to J. Milnor, "Hyperbolic components in Spaces of Polynomial Maps, Stony Brook I.M.S. Preprint 1992\#3.

[Pr] C. Preston, "What you need to know to knead", Advances Math. 78 (1989) 192-252.

[Re] M. Rees, "A minimal positive entropy homeomorphism of the 2-torus", J. London Math. Soc. (2) 23 (1981) 537-550.

[Ro] J. Rothschild, On the Computation of Topological Entropy, Thesis, CUNY, 1971.

[RS] J. Ringland and M. Schell, "Genealogy and bifurcation skeleton for cycles of the iterated two-extremum map of the interval", SIAM J. Math. Anal. 22 (1991) 13541371.

[RT] J. Ringland and C. Tresser, "A genealogy for finite kneading sequences of bimodal maps of the interval", Trans. Amer. Math. Soc. 347 (1995) 4599-4624.

[SI] A.N. Sharkovskii and A.F. Ivanov, " $C^{\infty}$-mappings of an interval with attracting cycles of arbitrary large periods", Ukrain. Mat. Zh. 35 (1983) 455-458.

[St] J. Stimson, Degree Two Rational Maps with a Periodic Critical Point, Thesis, Univ. Liverpool 1993. 
[Sw] G. Świątek, "Hyperbolicity is dense in the real quadratic family", Stony Brook I.M.S. Preprint 1992\#10.

[Ts] M. Tsujii, "A simple proof for monotonicity of entropy in the quadratic family", Preprint Hokkaido University, 1998.

[Y] Y. Yomdin, "Volume growth and entropy", Isr. J. Math. 57 (1987) 285-300. (See also " $C^{k}$-resolution of semialgebraic mappings", ibid. pp. 301-317.)

John Milnor

Institute for Mathematical Sciences SUNY

Stony Brook NY 11794-3651

jack@math.sunysb.edu
Charles Tresser

I.B.M.

P.O. Box 218

Yorktown Heights NY 10598

tresser@watson.ibm.com 